\title{
Measurement of the Neutron Electric Form Factor at $Q^{2}=0.8\left(\frac{\mathrm{GeV}}{\mathrm{c}}\right)^{2}$
}

\author{
by
}

Derek Glazier

Presented as a Thesis for the Degree of Doctor of Philosophy Nuclear Physics Group

Department of Physics and Astronomy

University of Glasgow

(C) Derek Glazier 2003 


\begin{abstract}
Nucleon form factors allow a sensitive test for models of the nucleon. Recent experiments utilising polarisation observables have resulted, for the first time, in a model-independent determination of the neutron electric form factor $G_{E}^{n}$.

This method employed an $80 \%$ longitudinally polarised, high intensity $(10 \mu \mathrm{A})$ electron beam $(883 \mathrm{MeV})$ that was quasi-elastically scattered off a liquid deuterium target in the reaction $D(\vec{e}, e \vec{n}) p$. A neutron polarimeter was designed and installed to measure the ratio of transverse-to-longitudinal polarisation using neutron scattering asymmetries. This ratio allowed a determination of the neutron electric form factor, $G_{E}^{n}$, free of the previous large systematic uncertainties associated with the deuterium wave function. The experiment took place in the A1 experimental hall at MAMI taking advantage of a high resolution magnetic spectrometer.

A detailed investigation was carried out into the performance of the neutron polarimeter. This utilised both analysis of the experimental data and the results of a Monte-Carlo simulation. The simulation required the creation of original models for polarised neutron scattering on hydrogen and carbon. These were able to successfully describe the experimental data giving confidence that analysis of the neutron polarisation is well understood.

The central work of this thesis is the analysis for a four-momentum transfer squared, $Q^{2}=0.79\left(\frac{\mathrm{GeV}}{\mathrm{C}}\right)^{2}$, model-independent measurement of $G_{E}^{n}$. It was found $G_{E}^{n}=0.047 \pm 0.009$ (stat) $\pm_{0.001}^{0.002}$ (sys).

The predictions of sophisticated nucleon models were then compared to the complete set of double polarisation data. The new data point reinforced that the Soliton model [1] gives the best parameterisation for the model-independent $G_{E}^{n}$ data.
\end{abstract}




\section{Acknowledgements}

The work described in this thesis was made possible with the help of a number of people, all of whom I would like to thank but I'm sure to miss out most so an extra special thanks to those not mentioned.

Firstly, my supervisors, Prof. Günther Rosner and Dr. John Annand, for the opportunity to work on the $G_{E}^{n}$ experiment and especially for their time, patience and good grammar while reading this thesis.

The experiment itself required a lot of work and preparation and I am grateful to all those involved for their efforts. In particular, Michael Seimetz, for his excellent work on the experimental setup and data analysis. Also Dr. Uli Müller, Prof. Hartmut Schmieden and John again, for running the experiment and for their help and discussion. Many thanks to Frank Klein for his valuable contributions and the rest of the A1 collaboration for their time and assistance during data-taking shifts.

Thanks to all the staff and students in the Glasgow Nuclear Physics Group who contributed in some manner to my work or social life. In particular, Dr. Ken Livingstone for computing assistance, Prof. Bob Owens for a variety of insights; Dr. Dan Watts, Duncan Middleton, Andreas Reiter and David Hamilton for their help with different aspects of the analysis.

I would also like to thank my family and Anna for their continuing support and hope they enjoy reading this thesis.

I'll dedicate this thesis to my Gran. 


\section{Declaration}

The data presented in this thesis were obtained as part of the Mainz Microtron (MAMI) A1 collboration with the Institut fur Kernphysik, Universität Mainz; Physikalisches Institut, Universität Bonn and the Nuclear Physics Experiment Group, Glasgow University. I participated fully in the preparation and excecution of the experiment. The analysis of the experimental data is my own work. This thesis was composed by myself.

Derek Glazier

September 2003. 


\section{Contents}

1 Introduction 1

2 Electron Scattering Formalism $\quad 4$

2.1 Elastic electron-nucleon scattering . . . . . . . . . . 4

2.1.1 Scattering Electrons off Pointlike Spin- $\frac{1}{2}$ Particles . . . . . 5

2.1.2 Scattering Electrons off Non-Pointlike Spin- $\frac{1}{2}$ Particles . . 7

2.1.3 Nucleon Electric and Magnetic Form Factors . . . . . . . 9

2.1.4 Rosenbluth Separation . . . . . . . . . . . . . . 9 9

2.1.5 Mean Squared Radii . . . . . . . . . . . . . . 10

2.1.6 Charge and Magnetisation Distributions . . . . . . . . 11

2.2 Electron-Deuteron Scattering . . . . . . . . . . . 13

2.2.1 Elastic Scattering . . . . . . . . . . . . . . 13

2.2.2 Quasi-Elastic Scattering . . . . . . . . . . . 16

2.3 Polarised electron scattering . . . . . . . . . . . . . . 19

2.3.1 Spin Dependent Free Electron-Nucleon Scattering . . . . . 19

2.3.2 Quasi-Elastic Spin Dependent Electron-Nucleon Scattering 21

3 Previous Form Factor Measurements $\quad 25$

$3.1 G_{E}^{n}$ Measurements . . . . . . . . . . . . . . . 25

3.1.1 Unpolarised Experiments . . . . . . . . . . . . 25

3.1.2 Double Polarisation Experiments . . . . . . . . 27

3.2 Measurements of $G_{M}^{n} \ldots \ldots \ldots . \ldots \ldots$

4 The $D\left(\vec{e}, e^{\prime} \vec{n}\right) p$ Experiment $\quad 34$

4.1 The Polarised Electron Beam . . . . . . . . . . . . 36

4.2 Møller Polarimeter . . . . . . . . . . . . . . . . . . . 37

4.3 Cryogenic Target . . . . . . . . . . . . . . 38

4.4 Electron Spectrometer . . . . . . . . . . . . . . . 39 
4.4.1 Spectrometer "A" Magnet Configuration . . . . . . . . . 40

4.4.2 Focal-plane detector system . . . . . . . . . . . . 41

4.5 Measuring the Neutron Polarisation . . . . . . . . . . . . . 44

4.5.1 Polarised Nucleon-Nucleon Scattering . . . . . . . . . . 44

4.5.2 Spin Precession Technique . . . . . . . . . . . . . . 49

4.5.3 Helicity Flipping . . . . . . . . . . . . . . . . 50

4.5.4 Isospin Symmetry . . . . . . . . . . . . . . 50

4.6 Neutron Polarimeter . . . . . . . . . . . . . . 51

4.7 Data Acquisition . . . . . . . . . . . . . . . . 52

5 Analysis $\quad \mathbf{5 6}$

5.1 Simulation of the Neutron Polarimeter . . . . . . . . . 56

5.1 .1 Polarimeter Geometry . . . . . . . . . . . 56

5.1 .2 Event Generation . . . . . . . . . . . . 57

5.1 .3 Particle Interactions . . . . . . . . . . . . . 59

5.1 .4 Light Response . . . . . . . . . . . . . . 62

5.1 .5 Data Analysis . . . . . . . . . . . . . 64

5.2 Neutron Polarimeter Calibrations . . . . . . . . . . 65

5.2 .1 QDC Pedestals ................. 66

5.2 .2 TDC conversion-gain calibration . . . . . . . . . 66

5.2 .3 Walk Correction . . . . . . . . . . . . . 66

5.2.4 Spatial Calibrations . . . . . . . . . . . 68

5.2.5 Time of Flight Calibrations . . . . . . . . . . 70

5.2.6 Energy Deposited in the First-Wall Scintillators . . . . . 72

5.3 Calculating Precession Angle . . . . . . . . . . . . . 72

5.3.1 Dipole-Field Integral . . . . . . . . . . . . . . 73

5.4 Event Reconstruction . . . . . . . . . . . . . . 75

5.4.1 Neutron Polarimeter Reconstruction . . . . . . . . . 75

5.4 .2 Electron Spectrometer . . . . . . . . . . 77

$5.4 .3 G_{E}^{n}$ Events .................... 77

5.5 Calculating Asymmetries . . . . . . . . . . . . . 82

5.5.1 Random Background Subtraction ........... 84

5.6 Nuclear Structure Corrections _. . . . . . . . . . . . 88

5.6 .1 Kinematic Correction . . . . . . . . . . . 90

5.6.2 Beyond Plane-Wave Impulse Approximation . . . . . . . . 92 
6 Results and Discussion $\quad 94$

6.1 Asymmetry versus Precession angle . . . . . . . . . . . . . 94

6.1.1 Fitting the spin precession . . . . . . . . . . . 94

6.1.2 Effect of Random Subtraction . . . . . . . . . . . 96

6.2 Proton Misidentification . . . . . . . . . . . . . . 96

6.2 .1 Proton signals . . . . . . . . . . . . . . . . . . . . 99

6.2 .2 Neutron Signals . . . . . . . . . . . . . . . . 101

6.3 Comparison of Simulated and Real Data . . . . . . . . . . 102

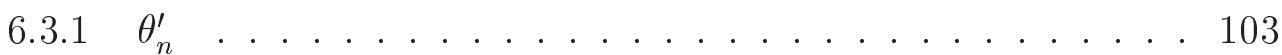

6.3.2 Kinematic Variable $R_{n p} \ldots \ldots \ldots \ldots$

6.3.3 Energy loss of the projectile . . . . . . . . . . 107

6.3.4 Conclusions on the simulation ............. 110

6.4 Final Result . . . . . . . . . . . . . . . . . . . 111

6.4.1 $G_{M}^{n}\left(Q^{2}=0.79\left(\frac{G e V}{c}\right)^{2}\right) \ldots \ldots \ldots . \ldots \ldots 112$

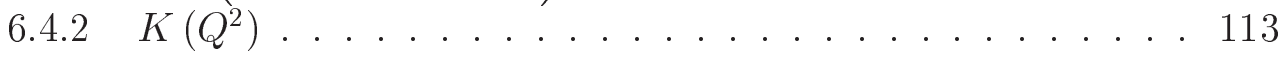

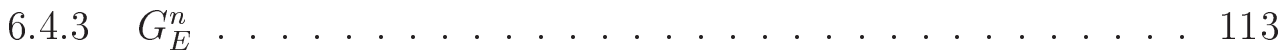

6.4.4 Systematic Uncertainties . . . . . . . . . . . 113

$6.5 G_{E}^{n}$ versus $Q^{2} \ldots \ldots \ldots \ldots \ldots \ldots$

6.5.1 Experimental Data and Phenomenological Fits . . . . . 115

6.5.2 Theoretical Models . . . . . . . . . . . . . . . . . . 119

6.6 Conclusions . . . . . . . . . . . . . . . 121

$\begin{array}{lr}\text { A Asymmetry Errors } & 123\end{array}$

A.1 Error on Random Distributions . . . . . . . . . . . . . . . 124

$\begin{array}{lr}\text { B Reaction Kinematics } & 127\end{array}$

B.1 Electron Scattering . . . . . . . . . . . . . . . 127

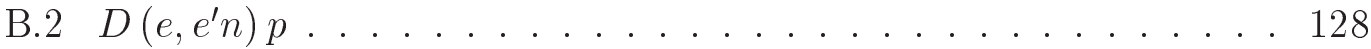

C Density Matrices, Structure Functions $\quad 130$

C.1 Virtual Photon Density Matrix . . . . . . . . . . . . . . 130

C.2 Nucleon Structure Functions . . . . . . . . . . . . . . . 130 


\section{Chapter 1}

\section{Introduction}

The last century saw incredible advancement in our understanding of the microscopic structure of the universe. Starting with Rutherford in 1911, who showed that on the scale of $10^{-10} \mathrm{~m}$ (much smaller than the wavelength of visible light) matter consisted of atoms with a central positive charge, which he named the "nucleus", surrounded by a cloud of negative electrons [2]. The nucleus itself was found to have dimensions less than $10^{-14} \mathrm{~m}$, but to contain $99.95 \%$ of the atomic mass. In 1919, Rutherford discovered the nucleus contained positively charged particles which he called "protons" [3]. The discovery of the neutron by Chadwick in 1932 [4] allowed the total mass and charge of the nucleus to be explained in terms of these two particles of similar mass, but with the proton having a positive charge exactly opposite to that of the electrons and the neutron zero charge. Collectively these two nuclear particles have become known as "nucleons". This gave a picture of the atom from a set of three elementary particles.

However, soon after the discovery of the neutron came a measurement of the proton magnetic moment by Frisch and Stern [5] which was not consistent with the prediction from Relativistic Quantum Theory. The prediction by Dirac implied that any truly elementary particle should have a magnetic moment determined by its charge and mass only. In 1939 Alvarez and Bloch confirmed that the neutron also had an anomalous magnetic moment and it became clear that the nucleons were not in fact pointlike elementary particles as the electron is, but instead have internal structure.

With the development of high energy electron beams advances were made in investigating nucleon structure. The interactions of an electron beam are dominated by the exchange of energy and momentum via a virtual photon, the carrier of the electromagnetic force. In the 1950's Hofstadter and others [6] found, 
using electron beams with energies of around $150 \mathrm{MeV}$ and scattering on light nuclei, that the cross sections obtained were not consistent with the Mott cross section for a point particle, but that the deviation could be explained by inclusion of a finite size term, as suggested by Rosenbluth [7], to account for a "meson cloud surrounding the proton". The extra term came to be written in terms of the Sachs electromagnetic form factors of the nucleon [8], which in this thesis are noted as $G_{E}$ and $G_{M}$. The electromagnetic form factors give a general parameterisation for the response of the nucleon to an electromagnetic probe.

The forties saw the discovery of new particles in cosmic rays. They were found to have a mass much greater than the electron but less than the nucleons and were given the name "mesons". The masses of the lightest mesons, known as pions, were of the right magnitude to be responsible for interactions between nucleons based on the exchange potential hypothesised by Yukawa in 1935. In 1964, GellMann layed down the quark model which noted that all nucleons and mesons, or more generally "hadrons", could be explained in terms of three elementary spin $-\frac{1}{2}$ particles with fractional charge and their anti-particles. He called these particles "quarks". It was later found that six quarks existed, the other three having masses much heavier than the original three, to go along with six leptons of which one is the electon. The modern picture of the nucleon is that it consists of three valence quarks ${ }^{1}$ and any number of quark-antiquark pairs. According to the theory of Quantum Chromodynamics the quarks interact through the strong interaction which is mediated by the exchange of "gluons".

The last fifty years have seen a continued effort into measuring the nucleon electromagnetic form factors using electron scattering techniques. The resolving power of the virtual photon, exchanged in the scattering process, is given by its negative squared four-momentum transfer, $Q^{2}$. The proton electromagnetic form factors were measured accurately as a function of $Q^{2}$ using a Rosenbluth separation technique on elastic electron-proton scattering cross sections. The form factors can then, via a Fourier transform, be related to the distribution of charge and magnetisation in the nucleon, thus providing an excellent testing ground for Quantum Chromodynamics.

The neutron form factors are altogether more difficult to measure. Neutrons have lifetimes of less than 15 minutes and so no static laboratory target exists

\footnotetext{
${ }^{1}$ The proton valence quarks are two "up" and one "down", while the neutron has one "up" and two "down". The "up" and "down" quarks are the two lightest. The third lightest is the strange quark, which is also present in the nucleon in virtual quark-antiquark pairs ("sea quarks").
} 
to study neutron interactions. Instead, neutrons bound in light nuclei have been used as a pseudo-free target, but even in the lightest nuclei this requires models of nuclear structure to extract the neutron form factors. These models and subtraction of the proton form factors lead to large systematic uncertainties in the measurements. In addition, neutrons are far more elusive to detect due to their zero net charge. Therefore calibration of the neutron detection efficiency yields another large source of systematic uncertainty. In particular, the neutron electric form factor has proven extremely difficult to determine as it is very small relative to its magnetic counterpart and as a result cannot be accurately separated using cross section measurements.

The development of polarised beams, targets and nucleon polarimetry over the last 10 years has given a new lease of life to form factor measurements. Polarisation observables are sensitive to the electromagnetic form factors. Exploiting the close relationship between experimental asymmetries and polarisation observables allows the determination of form factor ratios. This has, at last, lead to measurements of $G_{E}^{n}$ with relatively small systematic uncertainties.

It is the purpose of this work to determine $G_{E}^{n}$ at $Q^{2}=0.8\left(\frac{\mathrm{GeV}}{\mathrm{c}}\right)^{2}$ by measuring the recoil polarisation of neutrons quasi-elastically ejected from deuterium nuclei by a longitudinally polarised electron beam, i.e $D\left(\vec{e}, e^{\prime} \vec{n}\right) p$. The data analysed for this thesis was collected during April and May of 2001 in the A1 experimental Hall at MAMI. 


\section{Chapter 2}

\section{Electron Scattering Formalism}

\subsection{Elastic electron-nucleon scattering}

Electrons do not experience the strong nuclear force, their interaction with nucleons is dominated by the electromagnetic force. This interaction is accurately described to first order by the exchange of a virtual photon as shown in figure 2.1. Quantum Electrodynamics (QED) allows precise calculations of such physical processes using the Feynman rules, i.e. in principle we can calculate the cross section $(\sigma)$ and other observables for such a reaction. In practice, for an extended object such as a nucleon, a full calculation of $\sigma$ requires information on the internal distribution of charge and magnetisation which currently is not known precisely. On the other hand, as the electromagnetic interaction may be calculated almost exactly, the internal charge and current distribution may in principle be determined from experimentally measuring $\sigma$ or other observables.
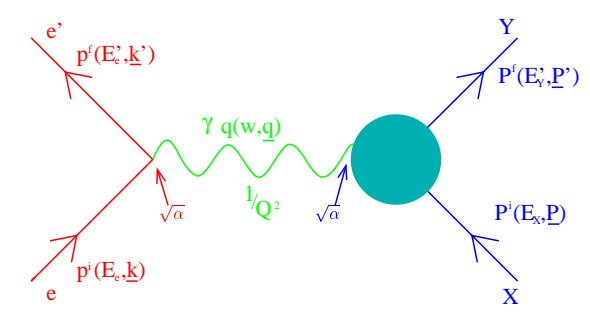

Figure 2.1: Electron scattering off a hadron via one photon exchange. In the case of elastic scattering $\mathrm{X}$ and $\mathrm{Y}$ must be the same particle. 


\subsubsection{Scattering Electrons off Pointlike Spin- $\frac{1}{2}$ Particles}

It was shown by Dirac that pointlike spin- $\frac{1}{2}^{1}$ particles have well defined magnetic moments [9]:

$$
\mu_{D}=\frac{Z e}{2 m}
$$

where $Z e$ and $m$ are the charge and mass of the particle respectively.

Taking $\mathrm{X}$ and $\mathrm{Y}$ (fig. 2.1) to be the same pointlike fermion i.e. elastic scattering, the differential cross section for electron scattering can be written as [10]:

$$
\frac{d \sigma}{d \Omega}=\frac{m_{e}^{2} M_{N}}{4 \pi^{2}} \frac{\left|\underline{p_{e}^{\prime}}\right|}{\left|\underline{p_{e}}\right|} \frac{\left|\overline{M_{f i}}\right|^{2}}{M_{N}+E-E_{e}^{\prime} \frac{\left|\underline{p_{e}}\right|}{\left|\underline{p_{e}^{\prime}}\right|} \cos \theta_{e}}
$$

where the initial and final four-vectors are shown in figure 2.1, $\theta_{e}$ is the scattering angle of the electron, $m_{e}$ and $M_{N}$ are the masses of the electron and fermion and $M_{f i}$ is the transition matrix between the initial and final states. In the one-photon exchange approximation, this is given by the product of the electron $\left(j_{E M}\right)$ and target $\left(J_{E M}\right)$ electromagnetic currents and a propogator $\frac{1}{Q^{2}}$. The propagator enters the matrix element to account for the electromagnetic interaction, $Q^{2}$ being the negative four-momentum squared of the exchanged virtual photon which carries the electromagnetic field. The $\frac{1}{Q^{2}}$ form arises from the Fourier transform of the electromagnetic potential in $r$-space, i.e it describes the electromagnetic field in momentum space and this inverse relationship means smaller distance scales are probed with photons of higher $Q^{2}$. In general for electromagnetic scattering,

$$
M_{f i}=\frac{1}{Q^{2}} j_{E M}^{\mu} J_{\mu}^{E M}
$$

with the matrix elements of the electron and target currents given by,

$$
\begin{gathered}
j_{E M}^{\mu}=\bar{u}\left(p^{f}, s^{f}\right) e \gamma^{\mu} u\left(p^{i}, s^{i}\right) \\
J_{\mu}^{E M}=\bar{u}\left(P^{f}, S^{f}\right) Z e \gamma^{\mu} u\left(P^{i}, S^{i}\right)
\end{gathered}
$$

Here $u(p, s)$ are the Dirac spinors (i.e. the wave function) for particles of fourvector momentum $p$ and spin $s$ which characterise the particles before and after

\footnotetext{
${ }^{1}$ Spin- $\frac{1}{2}$ particles like the electron, proton and neutron are collectively known as Fermions.
} 
the interaction. The $\gamma^{\mu}(\mu=0,1 \ldots 5)$ terms are the Dirac matrices, which operate at the vertices where the virtual photon starts and ends. In equations 2.2 and 2.3 they are operating on the initial and final wavefunctions. The strength of the interaction is given by the coupling constant ( $\alpha \equiv e^{2}$ in Gaussian units). $Z$ is the charge of the particle in units of charge $e$.

For the case of unpolarised scattering the spins are averaged over all possible initial states and summed over final states, so the squared transition matrix is then given by,

$$
\begin{aligned}
\left|\overline{M_{f i}}\right|^{2}= & \frac{Z^{2} e^{4}}{Q^{4}} \frac{1}{4} \sum_{\text {spins }}\left|\bar{u}\left(p^{f}\right) \gamma_{\mu} u\left(p^{i}\right) \bar{u}\left(P^{f}\right) \gamma^{\mu} u\left(P^{i}\right)\right| \\
= & \frac{Z^{2} e^{4}(4 \pi)^{2}}{2 m_{e}^{2} M_{N}^{2} Q^{4}} \times \\
& \left\{2 M_{N}^{2} E_{e} E_{e}^{\prime}-p^{f} \cdot p^{i}\left[M_{N}^{2}+M_{N}\left(E_{e}^{\prime}-E_{e}\right)\right]+m_{e}^{2} M_{N}^{2}\right\}
\end{aligned}
$$

It is instructive to consider the cross section for the case where the electron energy is very small compared to the rest mass of the target, so that the electron effectively scatters off a stationary point-like charged particle i.e. equivalent to being deflected in a Coulomb field. Taking $\frac{E_{e}}{M_{N}} \ll 1$ so that $E_{e}=E_{e}^{\prime} ;\left|\underline{p_{e}}\right|=\left|\underline{p_{e}^{\prime}}\right|$ and $\left(p^{i}\right)^{2}=\left(\underline{p}^{i}\right)^{2}$ yields :

$$
\begin{aligned}
\frac{d \sigma}{d \Omega} & \approx \frac{m_{e}^{2}}{4 \pi^{2}}\left|\overline{M_{f i}}\right|^{2} \\
& \approx \frac{Z^{2} e^{4}(4 \pi)^{2}}{2 m_{e}^{2}\left(\underline{p^{f}}-\underline{p^{i}}\right)^{4}}\left(2 E^{2}-\underline{p}^{f} \cdot \underline{p}^{i}+m_{e}^{2}\right) \\
& =\frac{Z^{2} \alpha^{2}\left(1-\beta^{2} \sin ^{2} \frac{\theta_{e}}{2}\right)}{4 \beta^{4} E_{e}^{2} \sin ^{4} \frac{\theta_{e}}{2}}
\end{aligned}
$$

when only terms up to order $M_{N}^{2}$ are considered in 2.4 and the relationships B.1B.3 from Appendix B have been used. This is just the Mott cross section $\left(\frac{d \sigma}{d \Omega}\right)_{M}$ for electron scattering in a Coulomb field.

The other case worth investigating is that for large electron energies which will be true for the present electron scattering experiment. At this relativistic limit, $\frac{m_{e}}{E_{E}} \ll 1$ and $\frac{\left|\underline{k}^{\prime}\right|}{|\underline{k}|} \rightarrow \frac{E_{e}^{\prime}}{E_{e}}$. The cross section in 2.1 becomes : 


$$
\frac{d \sigma}{d \Omega} \approx \frac{m_{e}^{2}}{4 \pi^{2}} \frac{E_{e}^{\prime}}{E_{e}} \frac{1}{1+\frac{2 E_{e}}{M_{N}} \sin ^{2} \frac{\theta_{e}}{2}}\left|\overline{M_{f i}}\right|^{2}
$$

Equation 2.4 can be rearranged using B.4-B.8 to give :

$$
\left|\overline{M_{f i}}\right|^{2}=\frac{\pi^{2} Z^{2} \alpha^{2}}{m_{e}^{2} E_{e} E_{e}^{\prime} \sin ^{4} \frac{\theta_{e}}{2}}\left(\cos ^{2} \frac{\theta_{e}}{2}+\frac{Q^{2}}{2 M_{N}^{2}} \sin ^{2} \frac{\theta_{e}}{2}\right)
$$

Inserting this into 2.6 gives :

$$
\frac{d \sigma}{d \Omega}=\left(\frac{d \sigma}{d \Omega}\right)_{M} f_{\text {rec }}^{-1}\left[Z^{2}+2 \frac{\mu_{F}^{2}}{e^{2}} Q^{2} \tan ^{2} \frac{\theta_{e}}{2}\right]
$$

where $\mu_{F}$ is the magnetic moment of the fermion. Here $\mu_{F}=\mu_{D}$, the Dirac magnetic moment. The recoil factor:

$$
f_{\text {rec }}=1+\frac{2 E_{e}}{M_{N}} \sin ^{2} \frac{\theta_{e}}{2}=\frac{E_{e}}{E_{e}^{\prime}}
$$

and

$$
\left(\frac{d \sigma}{d \Omega}\right)_{M}=\frac{\alpha^{2} \cos \frac{\theta_{e}}{2}}{4 E_{e}^{2} \sin ^{4} \frac{\theta_{e}}{2}} \text { for } \beta \rightarrow 1
$$

Thus the cross section for the case of relativistic electrons includes two additional terms. The first, $f_{r e c}$, is due to the recoiling of the target and causes the cross section to fall off with the scattered electron energy $E_{e}^{\prime}$. The terms in the square brackets contain separate magnetic and electrostatic contributions, the magnetic contribution being the second additional term to the non-relavistic case. For slow moving electrons $\beta \rightarrow 0, Q^{2} \approx 0$ so the magnetic force is negligible, $(Z e \underline{\beta} \times \underline{B}$ will be small $)$ but in the relativistic limit where $Q^{2}$ can be large it becomes significant.

Equation 2.7 also shows that an electron cannot interact with a neutral pointlike fermion which has zero charge and magnetic moment.

\subsubsection{Scattering Electrons off Non-Pointlike Spin- $\frac{1}{2}$ Parti- cles}

Protons and neutrons are both fermions with non-pointlike structure. Their measured magnetic moments are significantly different from their Dirac magnetic moments due to currents arising from motions of the internal partons. This measured value was the first indication of the composite nature of the nucleon whose 
magnetic moment is given by :

$$
\mu_{p}=\left(Z_{p}+\kappa_{p}\right) \frac{e}{2 M_{p}}, \quad \mu_{n}=\left(Z_{n}+\kappa_{n}\right) \frac{e}{2 M_{n}}
$$

where $Z_{p, n}=1,0$ is the nucleon charge and $\kappa_{p, n}=1.79,-1.91$ is called the anomalous magnetic moment ${ }^{2}$, i.e. the deviation from the Dirac magnetic moments. These are given in units of charge $e$ and the nuclear magneton, $\mu_{N}$, respectively.

The target current $J$ (eqn. 2.3) has to be altered to account for this internal structure. For the most general distributions of charge and magnetisation, the matrix elements for the nucleon current are [11],

$$
J^{\mu}=e \bar{u}\left(P^{\prime}\right)\left[\gamma^{\mu} F_{1}\left(Q^{2}\right)+\frac{i}{2 M_{N}} \sigma^{\mu \nu} q_{\nu} F_{2}\left(Q^{2}\right)+q_{\mu} F_{3}+\gamma_{\mu} \gamma_{5} F_{4}+q_{\mu} \gamma_{5} F_{5}\right] u(P)
$$

where the nucleon form factors $F_{i}$ have been introduced. This expression can be simplified in the case of pure electromagnetic currents by considering the conservation of parity, current and time reversal, which result in the form factors with no $Q^{2}$ dependence being zero, i.e. $F_{3}=F_{4}=F_{5}=0 . F_{1}\left(Q^{2}\right)$ and $F_{2}\left(Q^{2}\right)$ are known as the Dirac and Pauli form factors respectively. Using equation 2.11 to calculate the transition matrix elements (eqn. 2.4) and deriving the cross section for a nucleon with a general extended structure gives,

$$
\frac{d \sigma}{d \Omega}=\left(\frac{d \sigma}{d \Omega}\right)_{M} f_{r e c}^{-1} \quad\left\{\left[F_{1}^{2}\left(Q^{2}\right)+\frac{Q^{2}}{4 M_{N}^{2}} F_{2}^{2}\left(Q^{2}\right)\right]+\frac{Q^{2}}{4 M_{N}^{2}} 2\left[F_{1}\left(Q^{2}\right)+F_{2}\left(Q^{2}\right)\right]^{2} \tan ^{2} \frac{\theta_{e}}{2}\right\}
$$

Comparing this to equation 2.7, we can deduce that the charge and magnetic moment the virtual photon resolves is dependent on $Q^{2}$ :

$$
\begin{aligned}
& Z^{2} \rightarrow\left[F_{1}^{2}\left(Q^{2}\right)+\frac{Q^{2}}{4 M_{N}^{2}} F_{2}^{2}\left(Q^{2}\right)\right] \\
& \mu_{F}^{2} \rightarrow\left[F_{1}\left(Q^{2}\right)+F_{2}\left(Q^{2}\right)\right]^{2} \frac{e^{2}}{4 M_{N}^{2}}
\end{aligned}
$$

In the limit $Q^{2} \rightarrow 0$ internal structure is no longer resolved; the photon

\footnotetext{
${ }^{2}$ Which is actually the anomalous part of the anomalous nucleon magnetic moment.
} 
interacts with the nucleon as a whole and the cross section should then tend to equation 2.7. In this limit equation 2.13, 2.14 and 2.10 show that the form factors $F_{1}$ and $F_{2}$ tend to the charge $Z$ and anomalous magnetic moment $\kappa$ respectively.

\subsubsection{Nucleon Electric and Magnetic Form Factors}

In many cases it is more useful to use the Sachs form factors [8], which can be related directly to the charge and magnetisation distributions :

$$
G_{E} \equiv F_{1}-\tau F_{2} \quad \text { and } \quad G_{M} \equiv F_{1}+F_{2}
$$

$G_{E}\left(Q^{2}\right)$ and $G_{M}\left(Q^{2}\right)$ are known as the charge and magnetic form factors respectively and $\tau=\frac{Q^{2}}{4 M_{N}^{2}}$. Equation 2.12 can now be written in the Rosenbluth form [7],

$$
\frac{d \sigma}{d \Omega}=\left(\frac{d \sigma}{d \Omega}\right)_{M} f_{r e c}^{-1}\left\{\left[\frac{G_{E}^{2}\left(Q^{2}\right)+\tau G_{M}^{2}\left(Q^{2}\right)}{1+\tau}\right]+2 \tau G_{M}^{2}\left(Q^{2}\right) \tan ^{2} \frac{\theta_{e}}{2}\right\}
$$

In the limit $Q^{2} \rightarrow 0$ the electric and magnetic form factors are given by the integrated charge and magnetic moment of the nucleon, in units of $e$ and $\mu_{N}$ respectively:

$$
\begin{aligned}
G_{E}^{p}=1 & G_{M}^{p}=2.79 \\
G_{E}^{n}=0 & G_{M}^{n}=-1.79
\end{aligned}
$$

There are then four electromagnetic nucleon form factors: the proton and neutron electric and magnetic form factors, which will be represented by the symbols $G_{E}^{p}, G_{M}^{p}, G_{E}^{n}$ and $G_{M}^{n}$. It is sometimes useful to consider the nucleon as one particle with two different isospin states. In this case isoscalar and isovector form factors are used :

$$
G_{E, M}^{S}=\frac{1}{2}\left[G_{E, M}^{p}+G_{E, M}^{n}\right] \text { and } G_{E, M}^{V}=\frac{1}{2}\left[G_{E, M}^{p}-G_{E, M}^{n}\right]
$$

\subsubsection{Rosenbluth Separation}

The original method used to measure the nucleon form factors was to perform a Rosenbluth separation. Equation 2.16 can be written more generally in the form, 


$$
\frac{d \sigma}{d \Omega}\left(\frac{d \sigma}{d \Omega}\right)_{M}^{-1}=A\left(Q^{2}\right)+B\left(Q^{2}\right) \tan ^{2} \frac{\theta_{e}}{2}
$$

where $\left(\frac{d \sigma}{d \Omega}\right)_{M}$ is the cross section for scattering off a pointlike particle (eqn. 2.5) and $A\left(Q^{2}\right)$ and $B\left(Q^{2}\right)$ are the target structure functions (eqn. 2.16). This cross section can be measured experimentally for a fixed $Q^{2}$, but for a range of $\theta_{e}$ by varying the beam energy and using $Q^{2}=4 E_{e} E_{e}^{\prime} \sin ^{2} \frac{\theta_{e}}{2}$. Plotting $\frac{d \sigma}{d \Omega}\left(\frac{d \sigma}{d \Omega}\right)_{M}^{-1}$ against $\tan ^{2} \frac{\theta_{e}}{2}$ allows the structure functions to be determined from a straight line fit. The electromagnetic form factors can then be separated from the two structure functions.

\subsubsection{Mean Squared Radii}

Further nuclear structure information can be gained by considering again the limit $Q^{2} \rightarrow 0$. If the inverse Fourier transforms of equations 2.22-2.23 are expanded in $Q^{2}$ then the form factors can be expressed in terms of integral moments of the charge or magnetisation radii $\left\langle r_{E, M}^{k}\right\rangle$ :

$$
G_{E, M}\left(Q^{2}\right) \simeq\left\langle r_{E, M}^{0}\right\rangle-\frac{Q^{2}}{6}\left\langle r_{E, M}^{2}\right\rangle+\frac{Q^{4}}{120}\left\langle r_{E, M}^{4}\right\rangle-\ldots
$$

with,

$$
\left\langle r_{E}^{k}\right\rangle=\int r^{k+2} \rho(r) d^{3} r \text { and }\left\langle r_{M}^{k}\right\rangle=\int r^{k+2} \mu(r) d^{3} r, \text { for } k=0,2,4, \ldots
$$

Noting that $G_{E, M}(0)=\left\langle r_{E, M}^{0}\right\rangle$, we get again that the form factors at $Q^{2} \rightarrow 0$ are equal to the integral of the charge or magnetisation distributions, i.e. $Z$ or $\mu_{F}$. Taking the derivative of (2.19) with respect to $Q^{2}$ and ignoring terms left at order $Q^{2}$, we find that the the second moment $\left\langle r_{E, M}^{2}\right\rangle$, which is also known as the mean square radius, is given by,

$$
\left\langle r_{E, M}^{2}\right\rangle=-6\left(\frac{d G_{E, M}}{d Q^{2}}\right)_{Q^{2} \rightarrow 0}
$$

In this non-relativistic limit $\left\langle r_{E, M}^{2}\right\rangle$ is just the charge (magnetisation) weighted mean square position of the constituents. These radii have been accurately measured and it was found that the proton charge and magnetic radii and the neutron magnetic radii were very similar $[12,13,14]$ : 
$\left\langle r_{E}^{2}\right\rangle_{p}^{\frac{1}{2}}=(0.86 \pm 0.01) \mathrm{fm} ;\left\langle r_{M}^{2}\right\rangle_{p}^{\frac{1}{2}}=(0.86 \pm 0.06) \mathrm{fm} ; \quad\left\langle r_{M}^{2}\right\rangle_{n}^{\frac{1}{2}}=(0.873 \pm 0.011) \mathrm{fm}$

In the case of the neutron squared charge radius, experiments have shown it to be negative (i.e. the slope of $G_{E}^{n}$ versus $Q^{2}$ is positive at the origin). This result coupled with the zero net charge of the neutron implies a distribution with a central positive charge turning negative towards the surface. The most accurate measurement [15] which involved scattering thermal neutrons on the electron cloud of heavy atoms gave $\left\langle r_{E}^{2}\right\rangle_{n}=-(0.113 \pm 0.003 \pm 0.004) \mathrm{fm}^{2}$.

This interpretation of $\left\langle r_{E}^{2}\right\rangle_{n}$ being due to a static charge distribution in the neutron is still not certain. Using equation 2.15 to write equation 2.20 in terms of the Dirac and Pauli form factors :

$$
\left\langle r_{E}^{2}\right\rangle_{n}=-6\left[\left(\frac{d F_{1}^{n}}{d Q^{2}}\right)_{Q^{2} \rightarrow 0}-\frac{F_{2}^{n}(0)}{4 M_{n}^{2}}\right]
$$

We see that there is a contribution from the anomalous magnetic moment $\left(F_{2}^{n}(0)\right)$, which is known as the Foldy term [16] and arises as a relativistic correction associated with the neutrons anomalous magnetic moment. Its contribution is known $\left(F_{2}^{n}(0)=\kappa_{n}\right)$ to be $0.127 \mathrm{fm}^{2}$ which is very close to the measured $\left\langle r_{E}^{2}\right\rangle_{n}$. It would appear from this argument that the measured rest frame charge radius arises mainly from this Foldy term and not a static charge distribution. However using a valence quark model Isgur [17] showed that there is an additional contribution to $F_{1}^{n}$ that is not due to the intrinsic charge distribution and that this term exactly cancels the Foldy term allowing the charge radius of the neutron to be interpreted as due to a static charge distribution.

\subsubsection{Charge and Magnetisation Distributions}

To relate the electromagnetic form factors to the charge and magnetization distributions they must first be transformed into the Breit frame. This frame is defined as having zero energy transfer and so the photon four-momentum is given by $q=(0, \underline{q})$ and $Q^{2}=|\underline{q}|^{2}$. For the case of electron-nucleon scattering this is just the centre of mass frame. It was shown by Sachs [8] that the electromagnetic current in the Breit frame can be separated into electric and magnetic contributions, 


$$
J^{\mu}\left(\underline{q^{2}}\right)=Z e\left(G_{E}\left(\underline{q^{2}}\right),(\underline{\sigma} \times \underline{q}) G_{M}\left(\underline{q^{2}}\right)\right)
$$

with $\underline{\sigma}$ the Pauli matrices. Comparing equation 2.21 to the classical current density in $\underline{r}$ space,

$$
J_{c l}^{\mu}(\underline{r})=(e \rho(\underline{r}), \underline{\sigma} \times \underline{\nabla} \mu(\underline{r}))
$$

leads to the interpretation of the electromagnetic form factors in the Breit frame, as the Fourier transforms of the distributions of charge and magnetisation in configuration space.

$$
\begin{gathered}
\rho(r)=\frac{1}{e} \int G_{E}\left(\underline{q}^{2}\right) e^{-i \underline{q} \cdot \underline{r}} d^{3} \underline{q} \\
\mu(r)=\int G_{M}\left(\underline{q}^{2}\right) e^{-i \underline{q} \cdot \underline{r}} d^{3} \underline{q}
\end{gathered}
$$

An added complication to extracting the true charge and magnetisation distributions for a static nucleon arises from relativistic transforms between the Breit frame and rest frame of the nucleon. In fact a precise relativistic relationship between the Sachs form factors measured by electron scattering at finite $Q^{2}$ and the rest frame charge and magnetisation distributions does not exist [18]. This has two main reasons: first the transition densities between composite states of different momenta differ from the static densities in the rest frame; second the Lorentz boost of a composite system into a different frame depends on the interaction of its constituents. Different models of nucleon structure use different inversion procedures to account for this relativistic behaviour. Each is based on a Lorentz contraction of $Q^{2}$ to account for the difference in probed distance scales for the rest and Breit frames, due to the Lorentz contraction of the interacting wave packet (photon wavelength) as suggested by Licht and Pagnamenta [19],

$$
F\left(Q^{2}\right)=(1-\tau)^{\frac{1}{2}(1-n)} F_{\circ}\left(\frac{Q^{2}}{(1-\tau)}\right)
$$

here $\tau$ is defined as above, $F_{\circ}$ is the form factor before a relativistic correction and $\mathrm{n}$ is the number of particles in a cluster (nucleon). The choice of $\mathrm{n}$ relates to the model of nucleon structure being used.

Thus if the electromagnetic form factors of the nucleon are measured experi- 


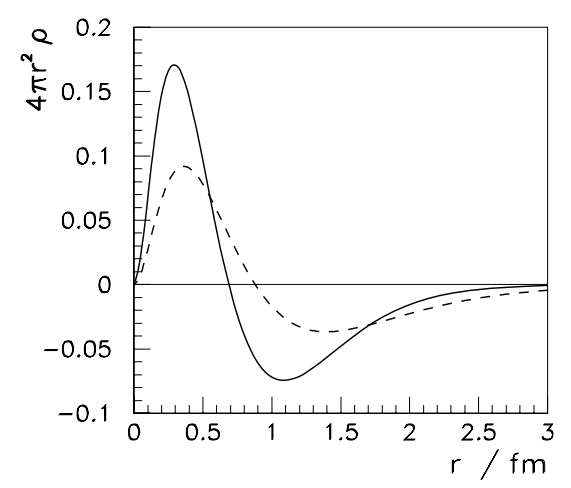

Figure 2.2: The neutron charge distribution from polarised [20] (full line) and the unpolarised [21] (dashed line) $G_{E}^{n}$ data.

mentally as a function of $Q^{2}$, the charge and magnetisation distributions of the nucleon may be determined. Figure 2.2, shows the neutron charge distribution using fits for both polarised and unpolarised experimental data, (sec. 3), using a non-relativistic transform.

\section{$2.2 \quad$ Electron-Deuteron Scattering}

Measuring proton form factors is relatively straightforward due to the existence of free proton targets e.g. liquid hydrogen. A complication to measuring the neutron form factors is the lack of a free target. Most previous experiments have used a deuteron target as a source of neutrons. As a result measurements of the neutron form factors rely on a knowledge of deuteron structure. This section will briefly show how the free nucleon form factors relate to the deuteron.

\subsubsection{Elastic Scattering}

A summary of well known integral deuteron properties are shown below :

\begin{tabular}{|c|c|}
\hline Mass & $1875.61339\left(\frac{\mathrm{MeV}}{\mathrm{c}}\right)^{2}$ \\
\hline Binding Energy & $2.224575 \mathrm{MeV}$ \\
\hline Spin parity & $1^{+}$ \\
\hline Isospin & 0 \\
\hline Quadrupole Moment & $0.28590 \mathrm{fm}^{2}$ \\
\hline Magnetic Moment & $0.857406 \mu_{N}$ \\
\hline
\end{tabular}


These properties restrict the deuteron ground state wave-function to be a combination of ${ }^{3} S_{1}$ and ${ }^{3} D_{1}$ states with wave functions $u(r)$ and $w(r)$ respectively. The relative contributions of the two states (the amplitude of $u$ and $w$ ) can be found by considering the deuteron magnetic and quadrupole moments. These wave functions are sensitive to the choice of the nucleon-nucleon potential (e.g. $[22,23,24])$, and as we see later this uncertainty in the wave function can lead to large systematic errors when extracting $G_{E}^{n}$ from electron-deuteron scattering data.

For electron-deuteron elastic scattering the cross section can be written similar to equations 2.16, and 2.18, showing that a Rosenbluth separation can be used to obtain the structure functions, $A\left(Q^{2}\right)$ and $B\left(Q^{2}\right)$ [25]:

$$
\frac{d \sigma}{d \Omega}=\left(\frac{d \sigma}{d \Omega}\right)_{M o t t}\left\{A\left(Q^{2}\right)+B\left(Q^{2}\right) \tan ^{2} \frac{\theta_{e}}{2}\right\}
$$

Clearly the deuteron, which is a composite system containing a proton and neutron is going to have different structure functions from individual nucleons. These structure functions are composed of three form factors instead of two, which is a result of it being a spin-1 particle. These form factors are known as charge monopole $\left(G_{C}\right)$, charge quadrupole $\left(G_{Q}\right)$ and magnetic dipole $\left(G_{M}\right)$ and are functions of $Q^{2}$ only. They are related to the structure functions through [26],

$$
\begin{aligned}
A & =G_{C}^{2}+\frac{8}{9} \eta^{2} G_{Q}^{2}+\frac{2}{3} \eta G_{M}^{2} \\
B & =\frac{4}{3} \eta(1+\eta) G_{M}^{2}
\end{aligned}
$$

with $\eta=\frac{Q^{2}}{4 M_{D}}$ playing a similar role to $\tau=\frac{Q^{2}}{4 M_{N}}$ in electron-nucleon scattering, where $M_{D}$ is the mass of the deuteron.

The deuteron form factors are then related to the nucleon isoscalar form factors of equation 2.17, and the deuteron wave-function [27] in the Non-Relativistic Impulse Approximation(NRIA) ${ }^{3}$ by:

\footnotetext{
${ }^{3}$ The impulse approximation neglects the effects of final-state interactions (FSI), isobaric and meson exchange currents (IC, MEC) etc. This is generally known as the Plane Wave Impulse Approximation (PWIA) which assumes both initial and final electrons are plane-wave states. The NRIA approximation is reasonable for $Q^{2}<1$ where relativistic corrections give small contributions[21]. There is also the Distorted-Wave Impulse Approximation which attempts to correct for FSI, MEC etc.
} 


$$
\begin{aligned}
G_{C} & =2 G_{E}^{S} C_{E}(q) \\
G_{Q} & =2 G_{E}^{S} C_{Q}(q) \\
G_{M} & =\frac{\mathrm{M}_{\mathrm{D}}}{\mathrm{M}_{\mathrm{p}}}\left(2 G_{M}^{S} C_{S}(q)+G_{E}^{S} C_{L}(q)\right)
\end{aligned}
$$

The functions $C(q)$ are structure integrals of the deuteron s- and d- state wave functions, $u(r)$ and $w(r)$ respectively. They are given by the Fourier-Bessel transforms of combinations of the spatial wave functions into momentum space. Explicitly,

$$
\begin{aligned}
C_{E} & =\int\left[u^{2}(r)+w^{2}(r)\right] j_{0}\left(\frac{q r}{2}\right) d r \\
C_{Q} & =\frac{3 \sqrt{2}}{2} \frac{1}{\eta} \int w(r)\left[u(r)-\frac{\sqrt{2}}{4} w(r)\right] j_{2}\left(\frac{q r}{2}\right) d r \\
C_{L} & =\frac{3}{2} \int w^{2}(r)\left[j_{0}\left(\frac{q r}{2}\right)+j_{2}\left(\frac{q r}{2}\right)\right] d r \\
C_{S} & =\int\left[u^{2}(r)-\frac{1}{2} w^{2}(r)\right] j_{0}\left(\frac{q r}{2}\right) d r \\
& +\frac{\sqrt{2}}{2} \int w(r)\left[u(r)+\frac{\sqrt{2}}{2} w(r)\right] j_{2}\left(\frac{q r}{2}\right) d r
\end{aligned}
$$

These equations show that $G_{C}$ is just given by a convolution of the proton and neutron charge structure (form factors) with the deuteron wave function, $C_{E}$ . The quadrupole form factor is dependent on the D-state wave function and would be zero without the D-state component $(w(r)=0)$, as for the predicted zero quadrupole moment for a pure S-state deuteron. $G_{C}$ may be considered as a naive charge distribution which is corrected by $G_{Q}$ for the effect of tensor forces between the states of different angular momentum (i.e. S,D) to give the overall charge contribution to the cross-section.

The magnetic dipole form factor has a similar simple interpretation to $G_{C}$ in the case of a pure S-state deuteron, being a convolution of the the sum of the nucleon magnetic form factors and the deuteron wave function. Again this has to be adjusted to account for the D-state contribution and tensor forces. 


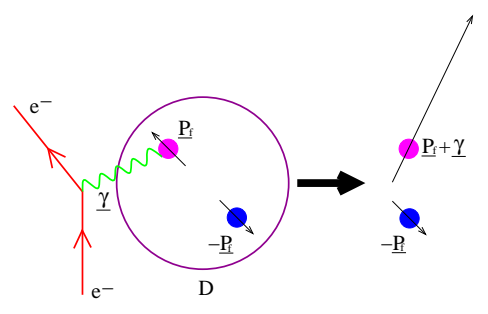

Figure 2.3: Schematic of quasi-elastic scattering, the momentum of the virtual photon is transferred to the struck nucleon only. The momentum of the rest of the nucleus is unchanged.

\subsubsection{Quasi-Elastic Scattering}

Quasi-elastic scattering is characterised by a virtual photon interacting with and ejecting one of the nucleons inside a nucleus, with the rest of the nucleus acting as a spectator. The struck nucleon will have an initial Fermi momentum $\underline{p}_{f}$ inside the nucleus with the other nucleon having the opposite to conserve momentum (fig. 2.3). In this approximation much of the physics simplifies to scattering from a free nucleon, particularly when the nucleon Fermi momentum is low compared to that of the virtual photon.

The two-fold differential cross-section for free electron-nucleon scattering (fig. 2.4) can be written in terms of the virtual photon density matrix $\rho$, and structure functions $f$. The matrix $\rho$, has elements that are functions of the scattered electron kinematics, while the structure functions are related to the nucleon charge and current. The cross section is [28] :

$$
\left(\frac{d \sigma}{d \Omega_{e}}\right)_{\text {free }}=S_{0}^{f r e e}=c\left[\rho_{L} f_{L}+\rho_{T} f_{T}+\rho_{L T} f_{L T}+\rho_{T T} f_{T T}\right] \text { where } c=\frac{\alpha}{6 \pi^{2} Q^{4}} \frac{E_{e}^{\prime}}{E_{e}}
$$

and the the subscripts $L$ and $T$ refer to the longitudinal and transverse components respectively. From Appendix C, we have $f_{L}=G_{E}^{2}, f_{T}=-2 \tau G_{M}^{2}$, $f_{L T}=f_{T T}=0$, and substituting for the virtual photon density matrix, (eqn. C.1), this equation reduces to equation 2.16 .

The general five-fold (the extra nucleon gives a further 3 degrees of freedom) cross section for quasi-elastic scattering from a nucleon in a deuteron (as shown in fig. 2.3) is, 


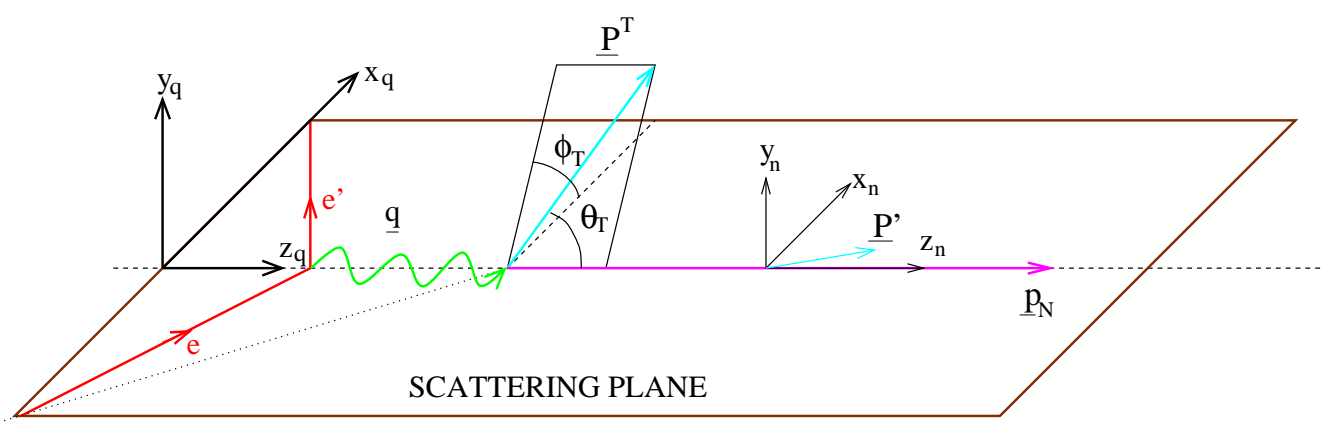

Figure 2.4: Free Scattering. The scattering plane is defined by the incoming and scattered electron. $\underline{z}_{q}=\underline{\widehat{q}} ; \underline{y}_{q}=\underline{\widehat{q}} \times \underline{\widehat{p}}_{e}$ and $\underline{x}_{q}=\underline{y}_{q} \times \underline{z}_{q}$. The $\left(\underline{x}_{n}, \underline{y}_{n}, \underline{z}_{n}\right)$ frame has the same axes directions. The orientation angles $\theta_{T}$ and $\phi_{T}$ of the target polarisation $\underline{P}^{T}$ are shown with respect to the virtual photon momentum and the scattering frame.

$$
\frac{d \sigma}{d E_{e}^{\prime} d \Omega_{e} d \Omega_{n p}^{C M}}=S_{0}=c\left[\rho_{L} f_{L}+\rho_{T} f_{T}+\rho_{L T} f_{L T} \cos \phi_{R}+\rho_{T T} f_{T T} \cos 2 \phi_{R}\right]
$$

where the structure functions now have deuteron structure terms as well as the nucleon form factors and $\phi_{R}$ is the angle between the electron scattering plane and the reaction plane defined by the two nucleons in the final state (fig. 2.5). For the Plane-Wave Born Approximation ${ }^{4}$ (PWBA), with a plane-wave final state $\left|p_{n p}^{C M}\right\rangle$ and a pure S-state deuteron $\left|\psi_{d}\right\rangle$, Arenhövel [28] showed the cross section becomes :

$$
\begin{gathered}
\left(\frac{d \sigma}{d E_{e}^{\prime} d \Omega_{e} d \Omega_{n p}^{C M}}\right)_{B o r n}=S_{0}=3 c a^{2}\left[4 M_{N}^{2} \rho_{L} \zeta_{E}^{2}+\frac{2}{3} \rho_{T} Q^{2}\left({ }^{0} \zeta_{M}^{2}+2 \cdot{ }^{1} \zeta_{M}^{2}\right)\right] \\
a=\frac{1}{4} \sqrt{\frac{p_{n p}^{C M}}{\pi M_{N}}} ; \\
\zeta_{E}=\alpha G_{E}^{p}+\beta G_{E}^{n} ; \quad{ }^{0} \zeta_{M}=\alpha G_{M}^{p}+\beta G_{M}^{n} ; \quad{ }^{1} \zeta_{M}=\alpha G_{M}^{p}+\beta G_{M}^{n}
\end{gathered}
$$

${ }^{4}$ The Plane-Wave Born Approximation combines the Born approximation : the electron exchanges one-photon in the scattering process; with the PWIA. 


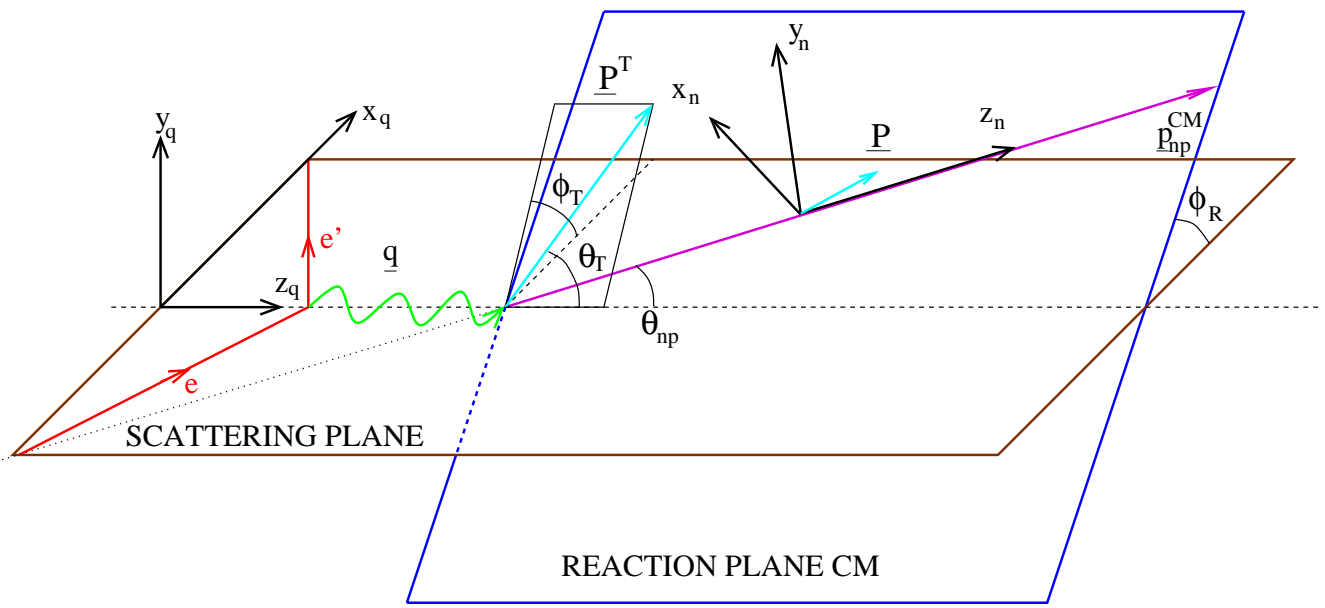

Figure 2.5: Quasi-elastic scattering. The scattering plane is defined by the incoming and scattered electron. The two frames for the polarisation are definded as $\underline{z}_{q}=\underline{\widehat{q}} ; \underline{\widehat{y}}_{q}=\underline{\widehat{q}} \times \underline{\hat{p}}_{e}$ and $\underline{x}_{q}=\underline{y}_{q} \times \underline{z}_{q} ;$ and now $\underline{z}_{n}=\underline{\hat{p}}_{n p}^{C M} ; \underline{y}_{n}=\underline{\widehat{p}}_{n p}^{C M} \times \underline{\widehat{q}}$ and $\underline{x}_{n}=\underline{y}_{n} \times \underline{z}_{n}$. The reaction plane is defined by the two nucleons in their centre-ofmomentum frame. The orientation angles $\theta_{T}$ and $\phi_{T}$ of the deuteron polarisation are shown with respect to the virtual photon momentum and scattering plane.

$$
\text { and } \alpha=\left\langle\underline{p}_{n p}^{C M}-\frac{1}{2} \underline{q}^{C M} \mid \psi_{d}\right\rangle ; \beta=\left\langle\underline{p}_{n p}^{C M}+\frac{1}{2} \underline{q}^{C M} \mid \psi_{d}\right\rangle
$$

Again the $f_{L T}$ and $f_{T T}$ structure functions are zero and the cross section is independent of $\phi_{R}$. At the quasi-elastic peak $\theta_{n p}=0$ (proton), $\pi$ (neutron) and $\underline{p}_{n p}^{C M} \pm \frac{1}{2} \underline{q}^{C M}=0$ (-proton, + neutron), where $\underline{p}_{n p}^{C M}$ is the CM momentum of the struck nucleon. So either the amplitude $\alpha$ or $\beta$ tend to zero for the case of scattering on the neutron or proton respectively. The quasi-elastic scattering cross section [28] is then,

$$
\left(\frac{d \sigma}{d E_{e}^{\prime} d \Omega_{e} d \Omega_{n p}^{C M}}\right)_{\text {Born }}^{q u a s i}=S_{0}^{q u a s i}=\left(\frac{d \sigma}{d \Omega_{e}}\right)_{\text {free }} a^{2} Q^{2}\left|\left\langle\underline{p}_{n p}^{C M} \pm \frac{1}{2} \underline{q}^{C M}=0 \mid \psi_{d}\right\rangle\right|^{2}
$$

where $\left|\left\langle\underline{p}_{n p}^{C M} \pm \frac{1}{2} \underline{q}^{C M}=0 \mid \psi_{d}\right\rangle\right|^{2}$ is the probability of either the proton or neutron having the same momentum direction in the final state as the virtual photon, which is again just the quasi-elastic condition: $\theta_{n p}=0, \pi$. In general the ejected nucleon will have a momentum direction differing slightly from that of the virtual photon, for scattering sufficiently close to the quasi elastic peak, $\left|\left\langle 0 \mid \psi_{d}\right\rangle\right|^{2}$ may be 
effectively replaced by the momentum density distribution of the struck nucleon inside the deuteron, $\left(\rho\left(\underline{p}_{f}\right)=\left|\left\langle\underline{p}_{f} \mid \psi_{d}\right\rangle\right|^{2}\right)$.

\subsection{Polarised electron scattering}

All the previous cross sections have been averaged over the initial and summed over the final spin states of the electron and target. However with recent advances in polarised beam and target technologies, investigation of spin dependent electron scattering has become experimentally feasible. Such experiments have become the favoured method for measuring the nucleon form factors, since for quasi-elastic scattering the knowledge of the nuclear wave function is not so important if ratios related to polarisation states, where the nuclear structure effects cancel to first order, are used.

\subsubsection{Spin Dependent Free Electron-Nucleon Scattering}

\section{Polarised beam and target}

The spin averaged cross section of equation 2.25 can be generalised in the onephoton exchange approximation to account for a longitudinally polarised electron beam and nucleon target with polarisation vector $\underline{P}^{T}$ (fig. 2.4),

$$
\left(\frac{d \sigma}{d \Omega_{e}}\right)_{\text {free }}^{\text {pol }}=c\left[\rho_{L} f_{L}+\rho_{T} f_{T}+\rho_{L T} f_{L T}+\rho_{T T} f_{T T}+h P_{e}\left(\rho_{L T}^{\prime} f_{L T}^{\prime}+\rho_{T}^{\prime} f_{T}^{\prime}\right)\right]
$$

where $h, P_{e}$ are the helicity and degree of longitudinal polarisation. The additional spin dependent structure functions are given by $f_{L T}^{\prime}=-\sqrt{2} \frac{q}{M} G_{E} G_{M}$ and $f_{T}^{\prime}=2 \tau Q^{2} G_{M}^{2}$. For experimental purposes it is convenient to write this cross section in terms of the electron-nucleon scattering asymmetries $\underline{A}_{e N}$. These are beam helicity-flip asymmetries of the cross section :

$$
\left(\frac{d \sigma}{d \Omega_{e}}\right)_{\text {free }}^{\text {pol }}=S^{\text {free }}(h, \underline{P})=S_{0}^{\text {free }}\left(1+h P_{e} \underline{A}_{e N} \cdot \underline{P}^{T}\right)
$$

giving,

$$
A_{e N}^{x_{i}}=\frac{1}{2 h P_{x_{i}}^{T} S_{0}^{\text {free }}}\left[S^{\text {free }}\left(h, P_{x_{i}}^{T}\right)-S^{\text {free }}\left(-h, P_{x_{i}}^{T}\right)\right]
$$


which can be experimentally determined by aligning the target polarisation along the $x_{i}^{q}$ axes, which are defined by the virtual photon momentum. $A^{z}$ is the asymmetry when the polarisation is aligned along the virtual photon direction and $A^{x(y)}$ when the polarisation is perpendicular to the virtual photon and parallel (perpendicular) to the scattering plane. Comparison with equation 2.30 allows the components of $\underline{A}_{e N}$ to be given in terms of the electromagnetic form factors:

$$
A_{e N}^{x}=-\frac{2 \rho_{L T}^{\prime} G_{E} G_{M}}{\rho_{L} G_{E}^{2}+2 \tau \rho_{T} G_{M}^{2}} ; A_{e N}^{y}=0 ; A_{e N}^{z}=\frac{2 \tau \rho_{T}^{\prime} G_{M}^{2}}{\rho_{L} G_{E}^{2}+2 \tau \rho_{T} G_{M}^{2}}
$$

For comparison with the Rosenbluth unpolarised cross section (eqn. 2.16) the full spin dependent cross section in terms of the spin averaged, $R_{u n}$ and spin dependent $R_{\text {pol }}$ nuclear response functions [29] is,

$$
\begin{gathered}
\left(\frac{d \sigma}{d \Omega_{e}}\right)_{f r e e}^{\text {pol }}=\left(\frac{d \sigma}{d \Omega_{e}}\right)_{M o t t} f_{\text {rec }}^{-1}\left[R_{u n}+h P_{e} P^{T} R_{\text {pol }}\right] \\
R_{\text {un }}=\left[\frac{G_{E}^{2}\left(Q^{2}\right)+\tau G_{M}^{2}\left(Q^{2}\right)}{1+\tau}\right]+2 \tau G_{M}^{2}\left(Q^{2}\right) \tan ^{2} \frac{\theta_{e}}{2} \\
R_{\text {pol }}=2 \tan ^{2} \frac{\theta_{e}}{2}\left[\tau \sqrt{\frac{1}{1+\tau}+\tan ^{2} \frac{\theta_{e}}{2}} G_{M}^{2} \cos \theta_{T}+\sqrt{\frac{1}{1+\tau}} G_{E} G_{M} \sin \theta_{T} \cos \phi_{T}\right]
\end{gathered}
$$

where $\theta_{T}$ and $\phi_{T}$ give the orientation of the target spin with respect to $\underline{q}$. The dependence of the polarised cross section on $G_{E}$ is then greatest when the nucleon spin is in the scattering plane, $\phi_{T}=0, \pi$ and perpendicular to the virtual photon, $\theta_{T}=\frac{\pi}{2}$. This can also be seen from $A_{e N}^{x}$ (eqn. 2.33)which requires the same orientation of the nucleon spin.

\section{Polarised beam and unpolarised target}

If a polarised beam is incident on an unpolarised target the virtual photon will transfer spin polarisation to the recoiling nucleon. The polarisation of the nucleon is related to the nucleon form factors in a similar manner as the scattering asymmetries.

The recoil polarisation $\underline{P}$ is given by analogy with equation 2.30 , 


$$
\begin{aligned}
\frac{d \sigma}{d \Omega_{e}} \underline{P} & =c\left[\rho_{L} \underline{g}_{L}+\rho_{T} \underline{g}_{T}+\rho_{L T} \underline{g}_{L T}+\rho_{T T} \underline{g}_{T T}+h P_{e}\left(\rho_{L T}^{\prime} \underline{g}_{L T}^{\prime}+\rho_{T}^{\prime} \underline{g}_{T}^{\prime}\right)\right] \\
& =S_{0}^{f r e e}\left(\underline{P}_{0}+h P_{e} \underline{P}^{\prime}\right)
\end{aligned}
$$

The z-axis of the polarisation frame is along the nucleon momentum and the y-axis is normal to the reaction plane (fig. 2.4). The splitting into beam polarisation dependent and independent parts separates the contribution from polarisation transfer and induced polarisation respectively [30]. The only non-zero polarisation structure functions, for elastic scattering, are $g_{L T}^{\prime x}=-\sqrt{2} \frac{q}{M_{N}} G_{E} G_{M}$ and $g_{T}^{\prime z}=$ $\frac{q^{2}}{2 M_{N}^{2}} G_{M}^{2}$, implying the induced polarisation $\underline{P}_{0}=0$ and

$$
P_{x}^{\prime}=-\frac{2 \rho_{L T}^{\prime} G_{E} G_{M}}{\rho_{L} G_{E}^{2}+2 \tau \rho_{T} G_{M}^{2}} ; P_{y}^{\prime}=0 ; \quad P_{z}^{\prime}=\frac{2 \tau \rho_{T}^{\prime} G_{M}^{2}}{\rho_{L} G_{E}^{2}+2 \tau \rho_{T} G_{M}^{2}}
$$

Thus a measurement of the recoil polarisation components will also give information on the nucleon form factors.

\subsubsection{Quasi-Elastic Spin Dependent Electron-Nucleon Scat- tering}

Quasi-elastic scattering approximates scattering on a nucleon inside a nucleus by elastic scattering with a nucleon which is not at rest. For polarised quasi-elastic scattering the spin of the nucleon inside the nucleus must also be known to allow investigation of the polarisation effects. In light nuclei such as $\mathrm{D}$ or ${ }^{3} \mathrm{He}$ the situation is relatively straightforward as both are largely in an S-state and so nucleon angular momentum contributions to the nuclear spin are small. For Sstate ${ }^{3} \mathrm{He}$ the Pauli Principal constrains the proton spins to be anti-aligned. The spin of the nucleus is then carried by the neutron and polarised ${ }^{3} \mathrm{He}$ can be used as a polarised neutron target if the spin of the nucleus is known. In practice ${ }^{3} \mathrm{He}$ is not a pure S-state and the protons do contribute to the net nuclear polarisation.

For an S-state deuteron the only way to have a net polarisation is with the spins of the proton and neutron aligned i.e. vector polarised. So again the neutron will have a polarisation that is the same as the nucleus to first order. Deviations of the neutron polarisation from the nuclear polarisation are much easier to calculate for the deuteron compared to ${ }^{3} \mathrm{He}$, as the former is relatively weakly bound and the two-body calculations are simpler than three-body.

For the case of quasi-elastic scattering on a deuteron target (fig. 2.5) the 
general spin dependent cross section can be written as,

$$
\begin{aligned}
\left(\frac{d \sigma}{d E_{e}^{\prime} d \Omega_{e} d \Omega_{n p}^{C M}}\right)^{p o l} & =S_{0}\left[1+h P_{e} A_{e}+P_{1}^{d} A_{d}^{V}+P_{2}^{d} A_{d}^{T}+P_{2}^{d} A_{d}^{T}+h P_{e}\left(P_{1}^{d} A_{e d}^{V}+P_{2}^{d} A_{e d}^{T}\right)\right] \\
& =S\left(h, P_{1}^{d}, P_{2}^{d}\right)
\end{aligned}
$$

where the beam, target and beam-target, vector and tensor asymmetries are defined by,

$$
\begin{aligned}
A_{e}= & \frac{1}{2 h S_{0}}[S(h, 0,0)-S(-h, 0,0)] \\
A_{d}^{V}= & \frac{1}{2 P_{1}^{d} S_{0}}\left[S\left(0, P_{1}^{d}, P_{2}^{d}\right)-S\left(0,-P_{1}^{d}, P_{2}^{d}\right)\right] \\
A_{d}^{T}= & \frac{1}{2 P_{2}^{d} S_{0}}\left[S\left(0, P_{1}^{d}, P_{2}^{d}\right)-S\left(0, P_{1}^{d},-P_{2}^{d}\right)\right] \\
A_{e d}^{V, T}= & \frac{1}{4 \sqrt{\frac{3}{2}} h P_{1}^{d} P_{2}^{d} S_{0}}\left[S\left(h, P_{1}^{d}, P_{2}^{d}\right)-S\left(-h, P_{1}^{d}, P_{2}^{d}\right)\right] \\
& \mp\left[S\left(h,-P_{1}^{d}, P_{2}^{d}\right)-S\left(-h,-P_{1}^{d}, P_{2}^{d}\right)\right]
\end{aligned}
$$

and $S_{0}$ is given by eqn. $2.26, P_{1,2}^{d}$ are the deuteron spin orientation parameters which are related to the vector and tensor polarisations.

For the Born approximation, the cross section simplifies as $A_{e}=A_{d}^{V}=A_{e d}^{T}=$ 0 . In addition for an S-state vector polarised deuteron $P_{1}^{d}=\sqrt{\frac{3}{2}} P_{z}$ and $P_{2}^{d}=$ $\sqrt{\frac{1}{2}} P_{z z}=0$, where $P_{z}$ is the magnitude of the deuteron vector polarisation and $P_{z z}$ the tensor polarisation. These relations mean that there is no change in the cross section if either the beam or target are unpolarised. The only remaining asymmetry $A_{e d}^{V}$, reduces to:

$$
\begin{aligned}
A_{e d}^{V} & =\frac{1}{4 \sqrt{\frac{3}{2}} h P_{e} P_{z} S_{0}}\left[S\left(h, P_{z}\right)-S\left(-h, P_{z}\right)-\left[S\left(h,-P_{z}\right)-S\left(-h,-P_{z}\right)\right]\right] \\
& =\frac{1}{2 \sqrt{\frac{3}{2}} h P_{e} P_{z} S_{0}}\left[S\left(h, P_{z}\right)-S\left(-h, P_{z}\right)\right] \\
& =-4 \sqrt{3} \frac{\rho_{T}^{\prime} \zeta_{M}^{2} \cos \theta_{T}+\sqrt{Q^{2}} M_{N} \rho_{L T}^{\prime} \zeta_{E} \cdot{ }^{1} \zeta_{M} \sin \theta_{T} \cos \phi_{T}}{6 M_{p}^{2} \rho_{L} \zeta_{E}^{2}+\rho_{T} Q^{2}\left[{ }^{0} \zeta_{M}^{2}+2^{1} \zeta_{M}^{2}\right]}
\end{aligned}
$$


where the $\zeta$ terms (eqn. 2.28) contain the nucleon form factors and the deuteron wave-function. As explained in section 2.2.2 the deuteron wave-function will factor out of the $\zeta$ terms in the quasi elastic limit $\left(\theta_{n p}=0, \pi\right)$. The wave-function will then cancel in $A_{e d}^{V}$ which will be independent of the NN potential as a result. In terms of the form factors, $G_{E, M}^{p(n)}$ for $\theta_{n p}=0(\pi)$, i.e. scattering off a proton (neutron), $A_{e d}^{V}$ is now given by,

$$
A_{e d}^{V}=-\frac{2}{3} \frac{\left[\rho_{T}^{\prime} G_{M}^{2} \cos \theta_{T}+2 \sqrt{2 \tau} \rho_{L T}^{\prime} G_{E} G_{M} \sin \theta_{T} \cos \phi_{T}\right]}{\rho_{L} G_{E}^{2}+2 \tau \rho_{T} G_{M}^{2}}
$$

For the case of deuteron polarisation directed perpendicular to the virtual photon direction, in the scattering plane i.e. $\theta_{T}=\frac{\pi}{2}, \phi_{T}=0, \pi, A_{e d}^{V} \simeq A_{e N}^{x}$, the beam target vector asymmetry reduces to the free eN asymmetry. The cross section is now given by,

$$
S\left(h, P_{z}\right)=S_{0}\left(1+h P_{e} A_{e d}^{V} P_{z}\right)
$$

\section{Polarised beam and unpolarised target}

Similar to equation 2.34 the polarisation components can be written, separating out the electron polarisation dependent part :

$$
\frac{d^{3} \sigma}{d E_{e}^{\prime} d \Omega_{e} d \Omega_{n p}^{C M}} \underline{P}=S_{0}\left(\underline{P}_{0}+h P_{e} \underline{P^{\prime}}\right)
$$

The polarisation $\underline{P}$ is given in the centre-of-momentum frame of the two nucleons, with the z-axis along the momentum of the struck nucleon, $\underline{p}_{n p}^{C M}$ and the y-axis parallel to $\underline{q} \times \underline{p}_{n p}^{C M}$, i.e. perpendicular to the reaction plane (fig. 2.5). When neglecting final state interactions this equation simplifies as $\underline{P}_{0}=0$ and for the Born approximation, $P_{y}^{\prime}=0$. The remaining components are given by,

$$
\begin{aligned}
& P_{x}^{\prime}(p / n)=4 \sqrt{2} \frac{a^{2} c q M}{S_{0}} \rho_{L T}^{\prime} \zeta_{E}\left( \pm^{0} \zeta_{M}^{2}+2 \cdot{ }^{1} \zeta_{M}^{2}\right) \\
& P_{z}^{\prime}(p / n)= \pm 2 \frac{c a^{2} q^{2}}{S_{0}}\left( \pm^{1} \zeta^{2}+2 \cdot{ }^{0} \zeta_{M} \cdot{ }^{1} \zeta_{M}\right)
\end{aligned}
$$

The \pm is required by convention as the direction of the quantisation axis (i.e $\underline{p}_{n p}$ ) will depend on whether a proton or neutron is hit. For the case of quasi-elastic scattering the $\zeta_{E, M}$ again tend to the product of the wave function 
amplitude, $\left|\left\langle 0 \mid \psi_{d}\right\rangle\right|^{2}$ and the nucleon form factors. $\left|\left\langle 0 \mid \psi_{d}\right\rangle\right|^{2}$ then factors out of the polarisation components, as it also factors out of the unpolarised cross section $S_{\circ}$ (sec. 2.2.2), so that the recoil polarisation components are the same as for free scattering equation 2.35 .

When there is a deviation from pure quasi-elastic scattering, the polarisation frames $\left(x_{q}, y_{q}, z_{q}\right)$ and $\left(x_{n}, y_{n}, z_{n}\right)$ no longer coincide (fig. 2.5). Therefore a rotation has to be performed to give the components in the $\left(x_{q}, y_{q}, z_{q}\right)$ frame where the simple relationships to the nucleon form factors apply. In the following the polarisation of the neutron is denoted $\underline{P}=\left(P_{x}, P_{y}, P_{z}\right)$. The superscripts $q$ and $n$ are used to indicate whether the polarisation vector is defined in the frame with either the z-axis along the virtual photon or neutron directions respectively i.e $\underline{P}^{q}$ or $\underline{P}^{n}$ (fig. 2.5). 


\section{Chapter 3}

\section{Previous Form Factor Measurements}

\section{1 $G_{E}^{n}$ Measurements}

\subsubsection{Unpolarised Experiments}

The original parameterisation for a non-zero $G_{E}^{n}\left(Q^{2}\right)$ was derived by Galster et al [31] from measurements of the electron-deuteron elastic cross-section at DESY (eqn. 2.24). For measurements with $\theta_{e}<15^{\circ}$ the structure function $B\left(Q^{2}\right)$ contributes less than $0.1 \%$ to the cross-section and so the measurement essentially gives structure function $A\left(Q^{2}\right)$, which is dependent on the four nucleon electromagnetic form factors and the deuteron wave function (sec. 2.2.1). Galster et al used a fit to the experimental $G_{E}^{p}$ data and assumed form factor scaling ${ }^{1}$ for $G_{M}^{p}$ and $G_{M}^{n}$, then fitted the $A\left(Q^{2}\right)$ data for a selection of deuteron wave functions using the parameterisation : $G_{E}^{n}\left(Q^{2}\right)=\frac{-\mu_{n} \tau}{(1+p \tau)} G_{E}^{p}\left(Q^{2}\right)$ with free parameter $p$. The best fit was found with the deuteron wave functions of Feshbach-Lomon [32] and $\mathrm{p}=5.6$. Figure 3.1 shows the Galster fits for different wave functions, showing a substantial systematic uncertainty in the parameterisation for the different nuclear models.

Separate measurements of the electron-deuteron elastic cross-section at Saclay [21] by Platchkov et al, led to another parameterisation of $G_{E}^{n}\left(Q^{2}\right)$. In this analysis corrections were made to $A\left(Q^{2}\right)$ to account for effects out with the impulse

\footnotetext{
${ }^{1}$ Form factor scaling assumes the same shape for the electromagnetic form factors, with the magnetic form factors scaled by the nucleon magnetic moment. This scaling works well at low $Q^{2}$ for $G_{E, M}^{p}$ and $G_{M}^{n}$, but does not apply to $G_{E}^{n}$.
} 


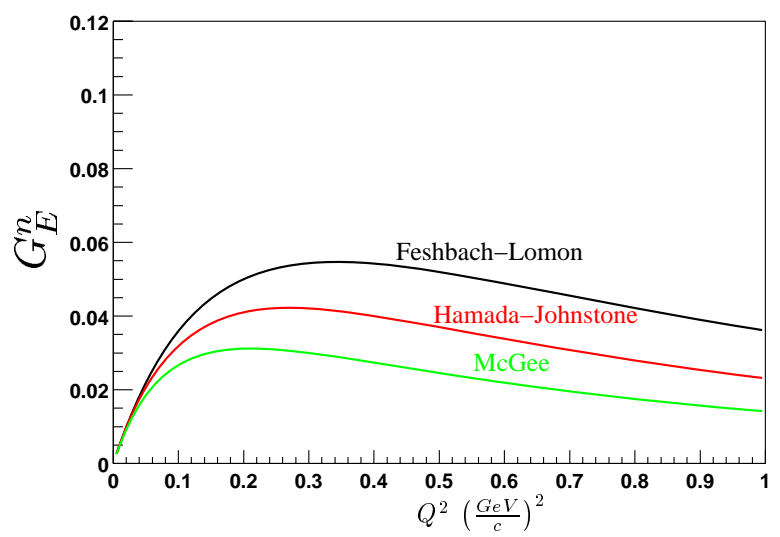

Figure 3.1: $G_{E}^{n}\left(Q^{2}\right)$ extracted from fits to $A\left(Q^{2}\right)$ data by Galster et al. The three lines show the results using the deuteron wave functions of FeshbachLomon[32] $(\mathrm{p}=5.6)$, Hamada-Johnstone[22] $(\mathrm{p}=10.7)$ and McGee[33] $(\mathrm{p}=19.7)$ respectively.

approximation, specifically relativistic corrections and meson exchange currents. To test the sensitivity of $G_{E}^{n}\left(Q^{2}\right)$ to theoretical models, a series of deuteron wave functions, distinguished by the form of the nucleon-nucleon potential, were used. An additional parameter $a$, was added to the parameterisation of Galster et al, which allowed a variation of the mean charge radius (i.e. the slope at $Q^{2}=0$ sec. 2.1.5): $G_{E}^{n}\left(Q^{2}\right)=\frac{-a \mu_{n} \tau}{(1+p \tau)} G_{D}\left(Q^{2}\right)^{2}$. Figure 3.2, shows the two-parameter fits $\left(\frac{\chi^{2}}{d o f} \simeq 1\right)$ for a selection of nucleon-nucleon potentials. The result shows a large sensitivity to the nucleon-nucleon potential, so again these parameterisations are very model dependent. Platchkov et al point out that only the Paris and RSC potential fits are consistent with the measured mean charge radius.

More recently Schiavilla and Sick [34] extracted $G_{E}^{n}$ from the deuteron quadrupole form factor $G_{Q}$. They determined $G_{Q}\left(\mathrm{Q}^{2}\right)$ from a fit to the electron-deuteron elastic scattering world data, using "flexible parameterisations" for the three deuteron form factors. This $G_{Q}^{e x p}\left(\mathrm{Q}^{2}\right)$ was compared to a theoretical calculation $G_{Q}^{t h}\left(\mathrm{Q}^{2}\right)$, which was given by the average of five independent calculations that initially used the Galster parameterisation for $G_{E}^{n}\left(Q^{2}\right)$. The theoretical calculation $G_{Q}^{t h}$ was then adjusted by altering $G_{E}^{n}$ until it agreed with $G_{Q}^{e x p}$. The resulting data for $G_{E}^{n}$ are shown in figure 3.3; the error bars include both the systematic

\footnotetext{
${ }^{2} G_{D}\left(Q^{2}\right)$ is known as the dipole form factor : $G_{D}\left(Q^{2}\right)=\frac{1}{\left(1+\frac{Q^{2}}{m_{D}^{2}}\right)}$, with $m_{D}^{2}=0.71$. The dipole form factor gives a good approximation to the proton electric form factor and when scaled by the their magnetic moments it also fits the nucleon magnetic form factors.
} 


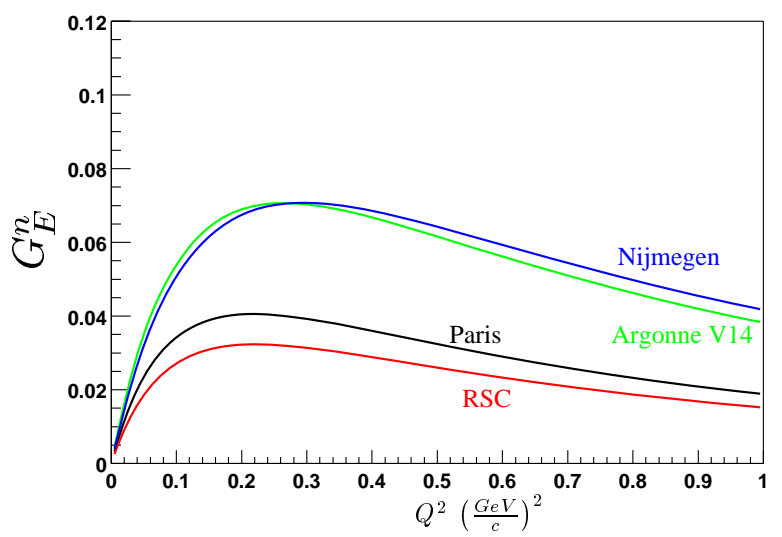

Figure 3.2: $G_{E}^{n}\left(Q^{2}\right)$ from Platchkov et al[21].

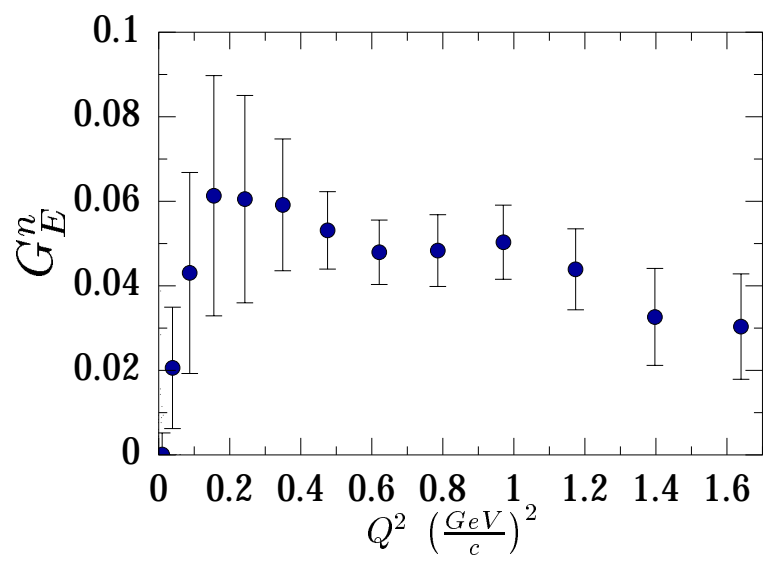

Figure 3.3: $G_{E}^{n}$ extracted by Schiavilla and Sick using the deuteron quadrupole form factor.

errors from the spread of theoretical predictions and the experimental uncertainty from the $G_{Q}^{e x p}\left(\mathrm{Q}^{2}\right)$ fit. This data agrees well with the parameterisation of Galster et al, but is systematically higher than the preferred parameterisation of Platchkov et al, which used the Paris potential for the deuteron wave function.

\subsubsection{Double Polarisation Experiments}

More recent experiments at MIT-Bates [35], NIKHEF [36]and MAMI [37][20], have taken advantage of polarised electron beams to use polarisation observables to extract information on $\mathrm{G}_{E}^{n}$. These also require either a polarised target as in the ${ }^{3} \overrightarrow{H e}\left(\vec{e}, e^{\prime} n\right) p p$ reaction, or alternatively information on the polarisation of 
neutrons scattered from unpolarised deuterium in the $D\left(\vec{e}, e^{\prime} \vec{n}\right) p$ polarisation transfer reaction, as discussed in section 2.3.2. Although big advances have been made in the theoretical description of two and three nucleon systems, the nuclear wave function is still not known exactly. Polarisation observables tend to show less dependence on the nuclear wave function than cross sections and so are able to circumvent the systematic uncertainties associated with the choice of nucleonnucleon potential models.

\section{$G_{E}^{n}$ through Polarisation Transfer}

There have been three previous $G_{E}^{n}$ measurements using the polarisation transfer technique. The corresponding $Q^{2}$ points were: $0.255\left(\frac{\mathrm{GeV}}{\mathrm{c}}\right)^{2}$ at MIT [35], $0.15\left(\frac{\mathrm{GeV}}{\mathrm{c}}\right)^{2}[20]$ and $0.34\left(\frac{\mathrm{GeV}}{\mathrm{c}}\right)^{2}$ [37] at MAMI. The technique for calculating $\mathrm{G}_{E}^{n}$ through the $D\left(\vec{e}, e^{\prime} \vec{n}\right) p$ reaction was suggested by Arnold, Carson and Gross [38], who used the results of [39] which showed the spatial components $\left(P_{x, y, z}^{q}\right)$ of the recoil neutron polarisation for free electron-neutron scattering to be :

$$
\begin{gathered}
P_{x}^{q}=-h P_{e} \frac{2 \sqrt{\tau(1+\tau)} \cdot \tan \left(\frac{\theta_{e}}{2}\right) G_{E}^{n} G_{M}^{n}}{\left(G_{E}^{n}\right)^{2}+\tau\left(G_{M}^{n}\right)^{2}\left(1+2(1+\tau) \tan ^{2}\left(\frac{\theta_{e}}{2}\right)\right)} \\
P_{y}^{q}=0 \\
P_{z}^{q}=h P_{e} \frac{2 \tau \sqrt{1+\tau+(1+\tau)^{2} \tan ^{2}\left(\frac{\theta_{e}}{2}\right)} \cdot \tan \left(\frac{\theta_{e}}{2}\right)\left(G_{M}^{n}\right)^{2}}{\left(G_{E}^{n}\right)^{2}+\tau\left(G_{M}^{n}\right)^{2}\left(1+2(1+\tau) \tan ^{2}\left(\frac{\theta_{e}}{2}\right)\right)}
\end{gathered}
$$

This is an equivalent result to that derived in section 2.3 and the coordinate system is the same as for figure 2.4, neutron frame.

For quasi-elastic scattering these results apply in the case of the Born approximation and parallel kinematics where the neutron is detected along the direction of the momentum transfer $\underline{q}$, as shown in section 2.3.2. In this case, where the effect of final state interactions is ignored, the proton effectively acts as a spectator and equations 3.1-3.3 imply, 


$$
\frac{P_{x}^{q}}{P_{z}^{q}}=-\frac{1}{\sqrt{\tau+\tau(1+\tau) \tan ^{2}\left(\frac{\theta_{e}}{2}\right)}} \cdot \frac{G_{E}^{n}}{G_{M}^{n}}
$$

A measurement of this polarisation ratio with the relevant kinematical variables and the previous experimental determination of $\mathrm{G}_{M}^{n}$ will then yield a value for $G_{E}^{n}$, independent of any model used for the deuteron wave function. Measuring the polarisation is described in detail in section 4.5.

In practice the finite phase space of the neutron and electron detector systems result in the measured neutrons deviating slightly from parallel kinematics. The polarisation ratio will in this case be subject to effects from the Fermi motion of the neutron bound in deuterium. Essentially this causes the polarisation axes to be rotated resulting in a deviation of the polarisation components from the free scattering case, $x_{i}^{q} \rightarrow x_{i}^{n}$. Also the Born approximation does not fully describe the response of the deuteron structure, particularly at low $Q^{2}$ where the amplitude of higher order corrections are greatly increased due to final state interactions (FSI), meson exchange currents(MEC), isobaric currents(IC) and relativistic effects. However, a previous analysis [20] of $G_{E}^{n}$ using the full model of Arenhövel, suggests that these effects are likely to be small at $Q^{2}=0.8\left(\frac{\mathrm{GeV}}{\mathrm{c}}\right)^{2}$, as they are $\sim 65,8 \%$ for $Q^{2}=0.15,0.34\left(\frac{\mathrm{GeV}}{\mathrm{c}}\right)^{2}$ respectively and will decrease further with increasing $Q^{2}$. Also, for the Fermi motion correction the average rotation of the polarisation axes is smaller for increasing $Q^{2}$ due to the relatively high momentum transfer compared with the Fermi momentum.

\section{$G_{E}^{n}$ through Polarised Beam and Target}

A double polarised $G_{n}^{E}$ measurement may also be realised by scattering polarised electrons off a polarised neutron target where analogous expressions to equations 3.1 and 3.3 can be found for the electron-nucleon polarisation asymmetries [28] $\underline{A}_{e N}$, replacing $P_{x}$ and $P_{z}$ with $A_{e N}^{x}$ and $A_{e N}^{z}$ respectively (sec. 2.3). The cross section for the case of quasi elastic scattering can be written, with $h, P_{e}$ the electron beam helicity and polarisation respectively and $\underline{P}^{T}$ the neutron polarisation, in the form (eqn. 2.31),

$$
S(h, \underline{P})=\frac{d \sigma}{d \Omega}=S_{0}\left(1 \pm h P_{e} \underline{A}_{e n} \cdot \underline{P}^{T}\right)
$$

where $S_{0}$ is the unpolarised electron-nucleon cross-section given by equation 2.16 
and the \pm indicates the helicity of the beam. The $\underline{A}_{e N}$ components can then be found by measuring the asymmetry with respect to beam helicity and aligning the target polarisation parallel(z) or perpendicular(x) to the virtual photon direction

$$
A_{e n}^{x, z}=\frac{1}{2 h} \frac{S\left(+h, P_{x, z}\right)-S\left(-h, P_{x, z}\right)}{S\left(+h, P_{x, z}\right)+S\left(-h, P_{x, z}\right)}
$$

Again as there are no free neutron targets the polarised neutron must be bound in a either $\mathrm{D}$ or ${ }^{3} \mathrm{He}$ and therefore corrections need to be applied to account for the polarisation of the neutron not being exactly that of the target, which is the polarisation measured in these experiments.

\section{Deuterium target}

There have been two previous $G_{E}^{n}$ measurements using polarised deuterium. The corresponding $Q^{2}$ points were: $0.21\left(\frac{\mathrm{GeV}}{\mathrm{C}}\right)^{2}$ [36], and $0.495\left(\frac{\mathrm{GeV}}{\mathrm{c}}\right)^{2}$ [40]. As has been discussed in section 2.3.2, for a deuterium target the vector beam-target asymmetry is measured, which is equivalent to the free beam-neutron asymmetry $\underline{A}_{e n}$ for quasi-elastic scattering on the neutron in the Born approximation. As for the recoil polarisation measurements higher order corrections need to be calculated to account for nuclear effects out with the Born approximation i.e. FSI, MEC etc, to correctly identify the free beam-nucleon asymmetry.

As shown in equation 2.33, $A_{e N}^{x}$ contains an interference term of the charge and magnetic form factors which allows $G_{E}^{n}$ to be extracted provided $G_{M}^{n}$ is known. In practice the actual value for $G_{E}^{n}$ has been extracted by reproducing the measured $A_{e d}^{V}\left(\theta_{d}=\frac{\pi}{2}, \phi_{d}=0\right) \simeq A_{e N}^{x}$, using the full Arenhövel model and iterating the value of $G_{E}^{n}$ until the comparison with the data is optimised. The dependence of the resulting $G_{E}^{n}$ on the deuteron model is more significant for these polarised target measurements as only one component of $A_{e d}^{V}$ is measured. In comparison the recoil polarisation methods measure the ratio of polarisation components where the higher order corrections of the deuteron model cancel to a greater degree. In the Hall A, JLAB $Q^{2}=0.5\left(\frac{\mathrm{GeV}}{\mathrm{c}}\right)^{2}$ measurement the contribution of FSI, MEC, IC and relativistic effects was estimated to be about $13 \%$ [40], compared to $8 \%$ for $Q^{2}=0.34\left(\frac{\mathrm{GeV}}{\mathrm{c}}\right)^{2}$ recoil polarisation experiment [20]. 


\section{${ }^{3} \mathrm{He}$ target}

There have been three previous $G_{E}^{n}$ measurements using a polarised ${ }^{3} \mathrm{He}$ target, the corresponding $Q^{2}$ points were: $0.385\left(\frac{\mathrm{GeV}}{\mathrm{C}}\right)^{2}[41]$ and $0.67\left(\frac{\mathrm{GeV}}{\mathrm{C}}\right)^{2}[42][43]$. For a polarised ${ }^{3} \mathrm{He}$ target the two protons are predominantly in the S-state, contributing a combined spin of zero. The polarisation is then carried to a high degree by the neutron. Previous experiments using targets of this type have measured the ratio of parallel and perpendicular asymmetries:

$$
\frac{A_{e n}^{x}}{A_{e n}^{z}}=\frac{A_{e n}\left(\theta_{T}=\frac{\pi}{2}, \phi_{T}=0\right)}{A_{e n}\left(\theta_{T}=0, \phi_{T}=0\right)}=\frac{1}{\sqrt{\tau+\tau(1+\tau) \tan ^{2} \frac{\theta_{e}}{2}}} \frac{G_{E}^{n}}{G_{M}^{n}}
$$

This is the same relationship as used for the recoil polarisation experiments, allowing a greater cancellation of the target nucleus structure. However, as there is a greater contribution from FSI and MEC in the more tightly bound ${ }^{3} \mathrm{He}$ nucleus larger, more complicated corrections are necessary to allow comparison of the measured asymmetry ratio with the free nucleon result above.

The $Q^{2}=0.385\left(\frac{\mathrm{GeV}}{\mathrm{c}}\right)^{2}$ measurement [41] was corrected in the Faddeev analysis of Golack et al [44] to account for the large FSI effects at low $Q^{2}$, the result was a $55 \%$ increase in $G_{E}^{n}$. The measurement at $Q^{2}=0.67\left(\frac{\mathrm{GeV}}{\mathrm{c}}\right)^{2}$ by [42] has recently been combined with new data at the same $Q^{2}$ [43] and corrected for FSI. Both data sets are consistent and the FSI correction resulted in a $3.4 \%$ increase in $G_{E}^{n}$.

\section{Results}

The results of all previous double polarisation experiments are shown in figure 3.4. This includes the $D\left(\vec{e}, e^{\prime} \vec{n}\right) p$ data from Mainz [37, 20] and MIT-Bates [35]; the $\vec{D}\left(\overrightarrow{e^{\prime}}, e^{\prime} n\right) p$ data from NIKHEF [36] and JLAB [40]; the ${ }^{3} \overrightarrow{H e}\left(\overrightarrow{e^{\prime}}, e^{\prime} n\right) p$ data from Mainz [42, 41, 44, 43]. Also shown are the Galster parameterisation and the Platchkov parameterisation with the Paris nucleon-nucleon potential.

\subsection{Measurements of $G_{M}^{n}$}

In previous double polarisation $G_{E}^{n}$ experiments which measure the ratio $\frac{G_{E}^{n}}{G_{M}^{n}}, G_{M}^{n}$ has been approximated by the dipole form factor: 


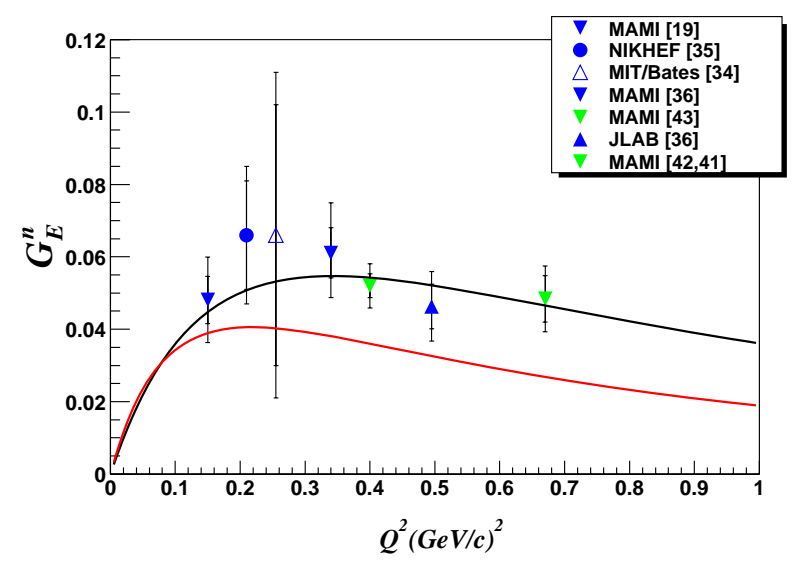

Figure 3.4: $G_{E}^{n}$ data from double polarisation experiments. From references (left to right) $[20,36,35,37,44,40,43,42]$. The green markers are from ${ }^{3} \mathrm{He}$ experiments and the blue from deuterium. Also shown are the Galster fit (black) and Platchkov fit (red).

$$
G_{M}^{n}=\mu_{n} G_{D}=\frac{\mu_{n}}{\left(1+\frac{Q^{2}}{0.71}\right)^{2}}
$$

Recent, more accurate experimental measurements of $G_{M}^{n}$ allow it to be distinguished from the dipole form factor, this gives a more accurate input for evaluating $G_{E}^{n}$.

The majority of these experiments have involved measuring the ratio of quasielastic neutron to proton cross sections $R=\frac{\sigma\left(e, e^{\prime} n\right)}{\sigma\left(e, e^{\prime} p\right)}$ in the $D\left(e, e^{\prime} N\right)$ reaction $[45,46,41,47]$. To first order this ratio is independent of nuclear binding effects as can be seen from the quasi-elastic cross section derived earlier (eqn. 2.29). Higher order effects can be calculated and corrected for to give the ratio in terms of the PWIA, where it is the same as for free electron-nucleon scattering. The well known elastic electron proton cross section is then used to extract the elastic neutron cross section, which is essentially given by $G_{M}^{n}$ as $G_{E}^{n}$ is very small in comparison. However, there have been significant discrepancies between the different experimental results. The ELSA results [47] give $G_{M}^{n}$ values 10-15\% higher than the other results. This discrepancy is due to the measurement of the neutron detection efficiency $\eta_{n}$ which is crucial in such a measurement as it is proportional to $R$. The ELSA experiment measured $\eta_{n}$ on site through the tagged $p\left(\gamma, \pi^{+}\right) n$ reaction, whereas the MAMI [46, 14] and NIKHEF [45] experiments transported their neutron detector to the PSI neutron beam facility which 


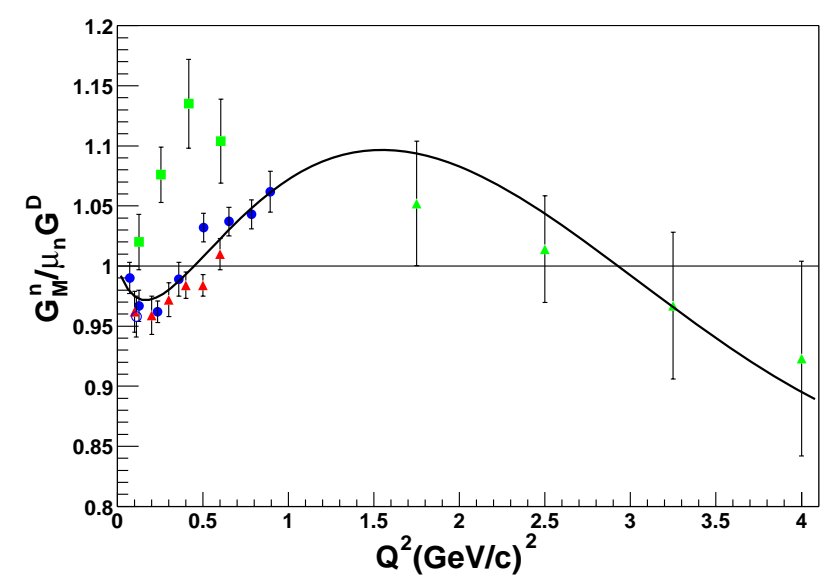

Figure 3.5: Ratio of the neutron magnetic form factor with the dipole form factor. Red triangles are from $D\left(e, e^{\prime} N\right)$ ratio Mainz/PSI [46, 14], green squares from ELSA $D\left(e, e^{\prime} N\right)$ [47], blue circles are from ${ }^{3} \overrightarrow{H e}(\vec{e}, e)[48,49]$ measurements and green triangles from $D\left(e, e^{\prime} N\right)$ Rosenbluth experiments [50]. The fit is the constant fraction parameterisation of [14].

employed the $p(n, p) n$ reaction. The latter method is considered to give the more reliable results.

The transverse asymmetry $A_{T^{\prime}}\left(=A_{e N}^{z}\right.$ in eqn. 2.32) of ${ }^{3} \overrightarrow{H e}(\vec{e}, e)$ quasielastic scattering has been used to determine $G_{M}^{n}$ with no dependence on $\eta_{n}$ $[48,49]$. As for the $G_{E}^{n}{ }^{3} \overrightarrow{H e}(\vec{e}, e)$ experiments, this technique uses the ${ }^{3} \overrightarrow{H e}$ as a polarised neutron target with the proton spins cancelling, thus the transverse asymmetry arises from scattering on the polarised neutron and is dominated by $G_{M}^{n}$.

Additional high $Q^{2}$ measurements have been made [50] using a Rosenbluth separation (sec. 2.1.4). Though the error bars are large they suggest that $G_{M}^{n}$ falls away from the dipole form factor at $Q^{2}>3\left(\frac{\mathrm{GeV}}{\mathrm{c}}\right)^{2}$.

The results from these experiments are shown in figure 3.5 with the result of the continued fraction parameterisation of Kubon et al [14]. The ELSA points are in disagreement with the other measurements and haven't been included in the fit. The fit shows $G_{M}^{n}$ deviating from the dipole form factor by up to $5 \%$ for $Q^{2}<1\left(\frac{\mathrm{GeV}}{\mathrm{c}}\right)^{2}$. Above this the quality of the data is poor and so the fit should not be taken too seriously. This parameterisation will be used in the final analysis to extract $G_{E}^{n}$. 


\section{Chapter 4}

\section{The $D\left(\vec{e}, e^{\prime} \vec{n}\right) p$ Experiment}

The 3-Spectrometer Facility (A1 Hall) [51], at the Mainz Microtron (MAMI), has been used to carry out the $D\left(\vec{e}, e^{\prime} \vec{n}\right) p$ experiment. The use of a high resolution magnetic spectrometer gave a higher precision measurement of the scattered electron momentum than had been achieved in previous Mainz $G_{n}^{E}$ experiments, which used a lead-glass calorimeter. This lead to a reduced systematic error in the virtual photon momentum and $Q^{2}$ and in addition the spectrometer gave a much cleaner separation of quasi-elastic scattering from non-elastic e-n processes and general background in the experimental hall.

The experiment measured the neutron electric form factor at $Q^{2}=0.3,0.6$ and $0.8\left(\frac{\mathrm{GeV}}{\mathrm{c}}\right)^{2}$, (the two lower $Q^{2}$ data have been analysed separately [52]). For the $Q^{2}=0.8\left(\frac{\mathrm{GeV}}{\mathrm{c}}\right)^{2}$ data an $883 \mathrm{MeV}$ beam with a current of $\sim 10 \mu \mathrm{A}$ was incident on a $5 \mathrm{~cm}$ thick liquid deuterium target.

Figure 4.2 , shows the experimental layout. The exclusive $\left(\vec{e}, e^{\prime} \vec{n}\right)$ reaction was tagged by detecting electrons and neutrons in coincidence. The electrons were momentum analysed in Spectrometer A (B and C were not used), while the neutrons were detected in a purpose built polarimeter which measured their scattering angles, time of flight and scattering asymmetries.

The electron scattering angle and incident energy determined the reaction $Q^{2}$, with the polarimeter centred along the direction of the virtual photon momentum to intercept as many of the quasi-elastically scattered neutrons as possible. The Fermi momentum of the neutron inside the deuteron will result in a deviation of the neutron recoil angle from that of the virtual photon, but the polarimeter is large enough to detect even neutrons which initially had high Fermi momenta. When deciding on the optimum kinematics it is also worth noting that the im- 


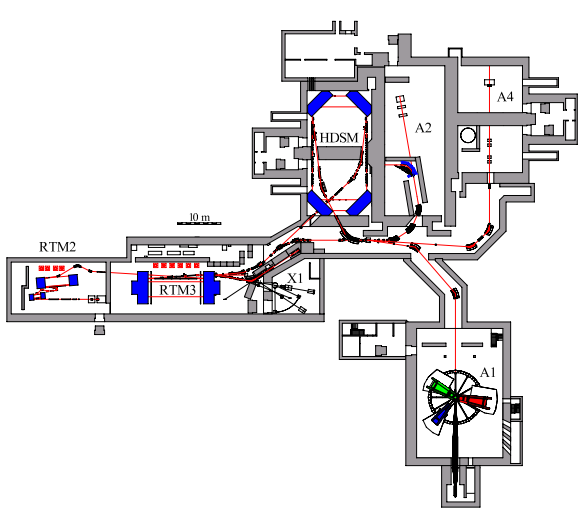

Figure 4.1: Layout of MAMI, showing the 3 racetrack microtrons (2 in RTM2 and 1 in RTM3) and the experimental halls A1, A2, A4, X1. The HDSM (Harmonic Double Sided Microtron) section is not curently operational, but will eventually allow an increase in beam energy to $1.5 \mathrm{GeV}$ in 2005 .

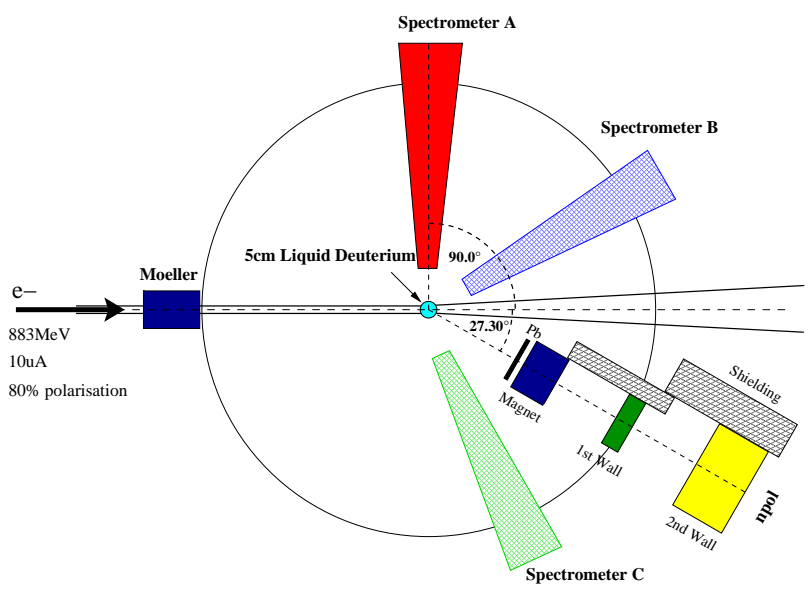

Figure 4.2: The A1 hall setup for the $Q^{2}=0.8\left(\frac{\mathrm{GeV}}{\mathrm{c}}\right)^{2}, D\left(\vec{e}, e^{\prime} \vec{n}\right) p$ experiment. 
portant x-component of the recoil neutron polarisation grows with increasing $\theta_{n}$, (neutron angle with respect to incident electron). However the overall figure of merit (event rate $)^{\frac{1}{2}} \times\left(\right.$ polarisation) decreases with increasing $\theta_{n}$, as the scattering cross section plummets as $\theta_{e}$ increases. To maximise the figure of merit, it was therefore desirable to place the polarimeter at as forward an angle as could be allowed by the hall geometry and fix the spectrometer angle to complement it. To achieve as small a $\theta_{e}$ as possible for a given $Q^{2}$ we had to operate at maximum beam energy. The set-up for the $Q^{2}=0.8\left(\frac{\mathrm{GeV}}{\mathrm{c}}\right)^{2}$ point required the detection of $453 \mathrm{MeV}$ electrons at an angle of $90^{\circ}$ to the beam direction. The complementary neutrons with energy $427 \mathrm{MeV}$ were then detected at $27^{\circ}$ on the other side of the beam-line. Since $883 \mathrm{MeV}$ is the absolute maximum beam energy for MAMI and $\sim 27^{\circ}$ the minimum angle the polarimeter can be positioned with respect to the beamline, $Q^{2}=0.8\left(\frac{\mathrm{GeV}}{\mathrm{c}}\right)^{2}$ is the maximum value obtainable for the $D\left(\vec{e}, e^{\prime} \vec{n}\right) p$ experiment at MAMI.

\subsection{The Polarised Electron Beam}

\section{Polarised electron source}

Polarised electrons [53] were obtained through photoelectron emission from the (III-V)-semiconductor crystal, GaAsP. A Ti:Sapphire laser was used to provide the polarised photons, as the wavelength of around $830 \mathrm{~nm}$ matches the energy gap between the valence and conduction bands of the GaAsP crystal. The linearly polarised beam from the laser was transformed to circularly polarised by a Pockel cell which acted as a quarter-wave plate. The degree of circular polarisation was better than $99.5 \%$ and the helicity was flipped by switching the polarity of the Pockel cell. Helicity flipping is important in the experiment to eliminate systematic instrumental asymmetries and the source was operated so that there was a $50 \%$ chance of a helicity flip each second.

This system provides a source of electrons longitudinally polarised to at least $75 \%$, although during our beam-time polarisations of up to $85 \%$ were observed.

\section{Electron Accelerator}

MAMI-B is a continuous-wave electron accelerator [54], which is hugely advantageous compared to previous pulsed beam linear accelerators. In coincidence experiments such as (e,e'n), the signal to random background ratio is propor- 
tional to the duty factor. For a given mean beam current the duty factor, defined as the ratio of the duration of an electron pulse to the time difference between two consecutive pulses, determines the instantaneous counting rates in the detector systems. For a continuous-wave machine, the duty factor is $100 \%$, while for the best pulsed LINAC beams it is typically $\sim 1 \%$, giving MAMI a factor 100 increase in the signal-to-random ratio.

To achieve continuous-wave operation MAMI uses low voltage gradients in its linear accelerating sections and thus many recirculations, accomplished in 3 consecutive racetrack microtrons, are necessary to achieve $\sim 900 \mathrm{MeV}$. Figure 4.1 shows a schematic layout of the facility. A $3.5 \mathrm{MeV}$ linear accelerator injects electrons, to the first racetrack, RTM1. In this first section the beam is accelerated to $14.35 \mathrm{MeV}$ after 18 re-circulations. In the second racetrack, RTM2, the beam reaches $180 \mathrm{MeV}$ after 51 re-circulations, before entering RTM3, where the electrons gain $7.5 \mathrm{MeV}$ each pass through the acceleration section. The beam can then be extracted by a movable deflection system after an even number of circulations, allowing electron energies $E_{e}(n)=\left(180.2+7.504 n-3.5 .10^{-5} n^{2} \pm 0.16\right)$ $\mathrm{MeV}$, up to the maximum of $883 \mathrm{MeV}$ allowed by the RTM3 magnets. Beams from pA to $100 \mu \mathrm{A}$ intensity, varied through focusing electrodes on the gun, may be delivered to any one of five experimental halls.

\subsection{Møller Polarimeter}

The Møller polarimeter used the asymmetry of $\vec{e}+\vec{e} \rightarrow e+e$ scattering. For a longitudinally polarised beam $\left(P_{B}\right)$ incident on a longitudinally polarised target $\left(P_{T}\right)$ the cross-section in the centre-of-mass frame at scattering angle $\theta$ is given by :

$$
\frac{d \sigma}{d \Omega}=\frac{d \sigma_{\circ}}{d \Omega}\left[1 \pm P_{T} P_{B} A_{Z Z}(\theta)\right]
$$

Where the unpolarised cross-section $\frac{d \sigma_{\circ}}{d \Omega}$ and the analysing power $A_{Z Z}(\theta)$ can be calculated to high precision using QED. The \pm , is dependent on the beam helicity, i.e. whether the beam polarisation is aligned parallel $(+)$ or anti-parallel (-) to the target polarisation. This allows measurement of a helicity dependent asymmetry from which the beam polarisation can be derived, provided the target polarisation is accurately known : 


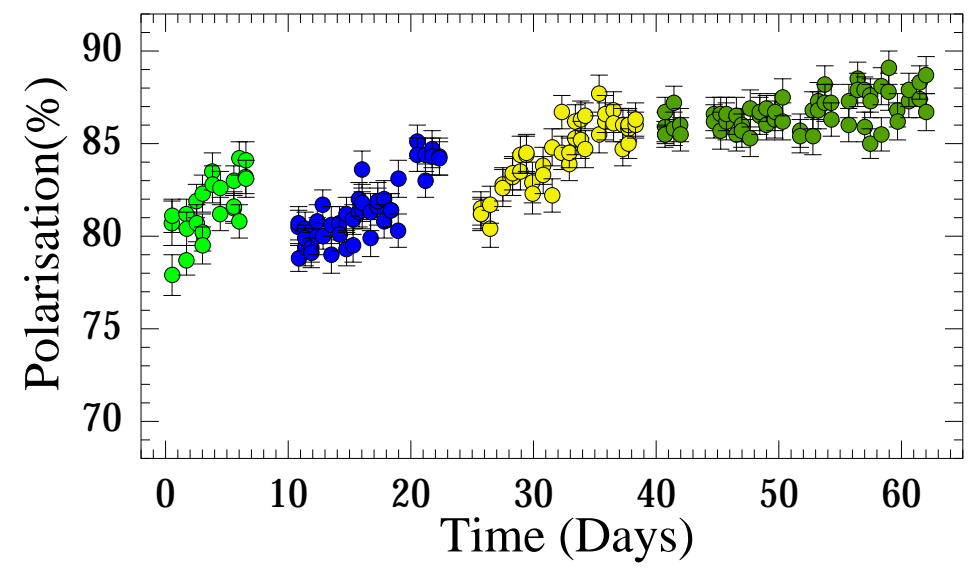

Figure 4.3: The electron beam polarisation for the $2001 G_{E}^{n}$ beamtime.

$$
\varepsilon=\frac{\frac{d \sigma^{+}}{d \Omega}-\frac{d \sigma^{-}}{d \Omega}}{\frac{d \sigma^{+}}{d \Omega}+\frac{d \sigma^{-}}{d \Omega}}=A_{Z Z}(\theta) P_{B} P_{T}
$$

The A1 Møller polarimeter contained a $6 \mu \mathrm{m}$ thick magnetised iron foil. The spins of the atomic electrons were aligned parallel to the beam direction using a 4-Tesla superconducting solenoid, giving a target polarisation of $8.05 \%$. A quadrupole magnet focused the Møller electrons through a collimator, which accepted azimuthal angles of $\pm 12^{\circ}$ about the vertical scattering plane. Electrons that passed through the collimator were then deflected in to a dipole field to hit two lead glass detectors. The collimator, dipole field and detectors were carefully configured so that only a Møller electron pair gave a signal in both detectors. Counting this coincidence signal for different beam helicities then allowed the determination of the beam polarisation by using equation 4.1 .

Figure 4.3 shows the beam polarisation as monitored by the Møller polarimeter during the $2001 G_{E}^{n}$ beamtime. The polarisation increased with time which is a typical aging phenomenon of GaAsP crystals.

\subsection{Cryogenic Target}

During the experiment both a liquid Hydrogen (LH2) and a liquid Deuterium (LD2) target were employed. The Hydrogen target was required for calibration purposes and the Deuterium for the $D\left(\vec{e}, e^{\prime} \vec{n}\right) p$ data-taking. A schematic of the cryo-target is shown in figure 4.4. The beam was incident on a $5 \mathrm{~cm}$ long target 


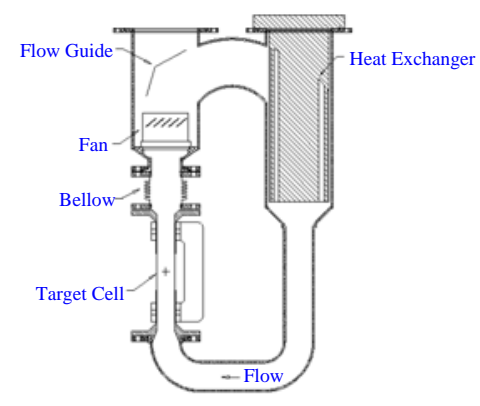

Figure 4.4: The A1 cryo-target.

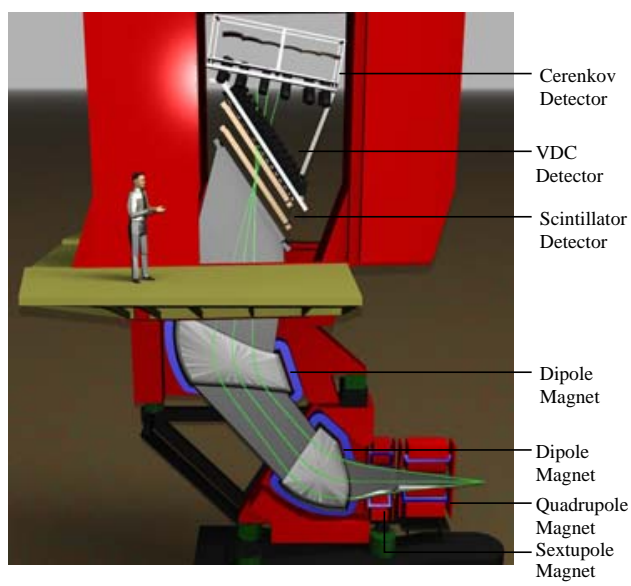

Figure 4.5: Schematic diagram of Spectrometer A, A1 Hall, Mainz. (Picture H.Merkel).

cell containing LH2 or LD2. The scattering chamber was connected by a transfer line shielded by liquid nitrogen, to a LH2 source which had been cooled to $21 \mathrm{~K}$ by a Philips machine cryo-generator, which operates at a power of approximately 60 W [55]. This then cooled the liquid target in a heat exchanger. The target liquid was circulated through the cell and the beam position was continuously rastered, with an amplitude of $6 \mathrm{~mm}$, to minimise boiling due to localised heating by the beam.

\subsection{Electron Spectrometer}

The A1 hall at MAMI contains 3 high resolution magnetic spectrometers (fig. 4.5), which can be used for the detection of both positively and negatively charged particles [51]. Magnetic spectrometers rely on measuring the bend radius of 


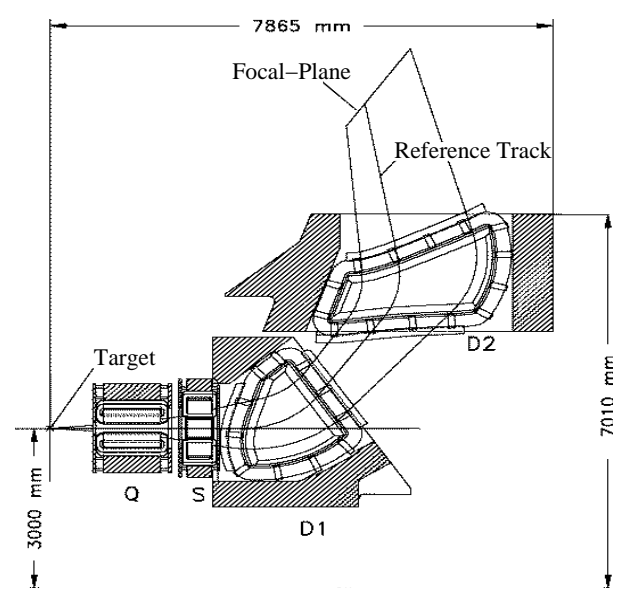

Figure 4.6: Layout of spectrometer magnets.

charged particles in a magnetic field to determine the particle momentum. The A1 spectrometers were developed to take advantage of the high duty-factor electron beam for investigations of hadronic and nuclear structure. Due to the small cross sections intrinsic with electron scattering reactions this requires that the spectrometers have as large a solid angle and momentum acceptance as possible while still providing a small momentum resolution $\left(\sim 10^{-5}\right)$. This in turn requires that the particle track is measured across the focal plane to allow accurate momentum reconstruction. A detector system consisting of high position resolution wire chambers for tracking, fast plastic scintillation detectors for timing resolution and a threshold Cerenkov gas detector for particle identification has been employed to allow accurate event reconstruction.

For our experiment Spectrometer A was used to detect the scattered electron and reconstruct its four-momentum, thus giving an accurate measure of the virtual photon momentum and the reaction $Q^{2}$.

\subsubsection{Spectrometer "A" Magnet Configuration}

The layout of the magnets is shown in figure 4.6. It consists of an entrance quadrupole(Q), followed by a sextupole(S) and two dipoles(D1,D2).

The quadrupole magnet defocuses the incident electrons in the dispersive plane to allow higher momentum resolution. In addition it is used to reduce high-order optical aberrations. The sextupole corrects for the larger second order aberrations, allowing the particle track to be accurately reconstructed. Further optical corrections are implemented in software using information from the focal- 
plane tracking detectors.

The dipoles are responsible for bending the track of the charged particle allowing its momentum to be analysed. The homogeneity of the dipole field has to be of the same order as the required momentum resolution $\left(10^{-5}\right)$. In Spectrometer $\mathrm{A}$ the dipoles can operate a maximum induction of $1.5 \mathrm{~T}$. The deviation from a reference track (i.e. with known momentum) allows the determination of the particle momentum. The reference track lies in the symmetry plane of the spectrometer and is defined as the trajectory of a particle that emerges with momentum $p_{\circ}$ from the centre of the target through the centre of the spectrometer acceptance.

The spectrometer is symmetric with respect to a mid-plane, assumed to be the $\mathrm{x}-\mathrm{z}$ plane, to which the magnetic field is perpendicular everywhere. The $\mathrm{z}$ axis is defined by the reference particle trajectory and is the direction of its momentum. The $\mathrm{x}$-axis is then perpendicular to both the $\mathrm{z}$ axis and the magnetic field and the $y$ axis forms a right-handed coordinate frame with the other two. The trajectory of any particle is described by its deviation from the reference particle in position $(\mathrm{x}, \mathrm{y})$ at the focal plane, direction of the track and momentum $\left(\delta=\left(p-p_{o}\right) / p_{o}\right)$, where $p_{o}$ is the momentum of the reference particle.

\subsubsection{Focal-plane detector system}

The spectrometer detector systems are used to measure the trajectory of the particle after momentum analysis with an accuracy that permits the reconstruction of the reaction vertex, the particle momentum and the in-plane, out-of-plane emission angles by tracing the particle trajectory back to the target. They are also used to identify the type of charged particle being analysed. This information can then be used, in the case of an electron, to accurately determine the four-momentum of the virtual photon that is exchanged in the target and thus the reaction $Q^{2}$.

A diagram of the detectors is shown in figure 4.7. They consist of three main components : four vertical drift chambers (VDCs) for tracking the electron to allow the reconstruction of its momentum and reaction vertex. Two segmented planes of plastic scintillation detectors provide a trigger signal with $\sim 1$ ns timing resolution and particle identification. Threshold Cerenkov detectors allow the separation of electrons from negatively charged pions. 


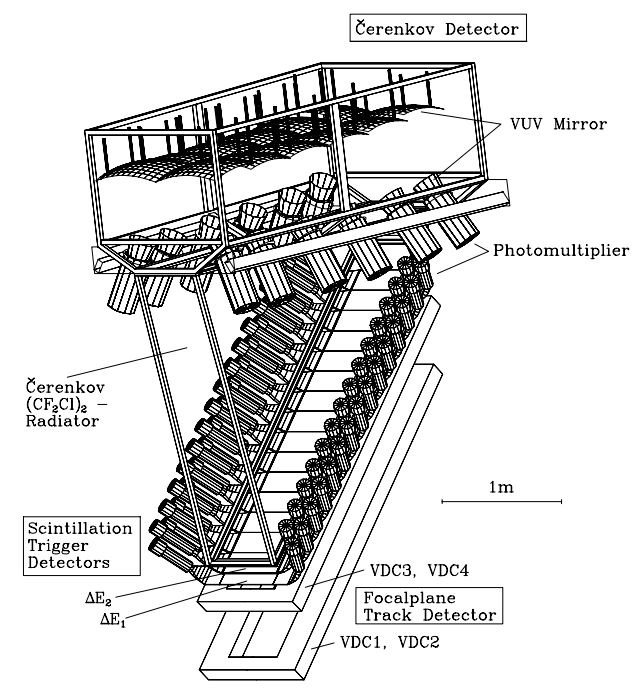

Figure 4.7: The focal-plane detector system for Spectrometer A.

\section{Vertical Drift Chambers}

Each drift chamber consists of four planes of VDCs, each constructed from several hundred drift cells, with a single VDC plane shown schematically in figure 4.8 - The cells are confined by two cathode planes positioned $24 \mathrm{~mm}$ apart, which typically sit at a high voltage of around $6 \mathrm{kV}$. The cell wires lie in a plane parallel to the cathodes and alternates between signal and potential wires with diameters 15 and $50 \mu \mathrm{m}$ respectively. The distance between each signal and potential wire is $2.5 \mathrm{~mm}$. The VDC is filled with a mixture of $49.25 \%$ argon and $49.25 \%$ isobutane with $1.5 \%$ ethanol to minimise aging.

A charged particle traversing the VDC (fig. 4.8) will ionise the gas molecules and the electric field between the potential wires and cathode planes will then cause the electrons to drift towards the wires ionising more molecules on the way. The drift-time for the ionised electrons to reach the signal wires, with the reference time coming from the scintillator detectors, is related to the distance of the ionisation from the wire. The measurement of drift time and hence this distance in consecutive cells of the VDC along the ionising track allows reconstruction of the $\mathrm{x}-\mathrm{y}$ hit coordinates. Successive drift chamber planes will each yield an $\mathrm{x}-\mathrm{y}$ position allowing reconstruction of the particle track. Knowledge of the spectrometer magnets imaging properties then allow a calculation of the particle momentum at the target as explained below. 


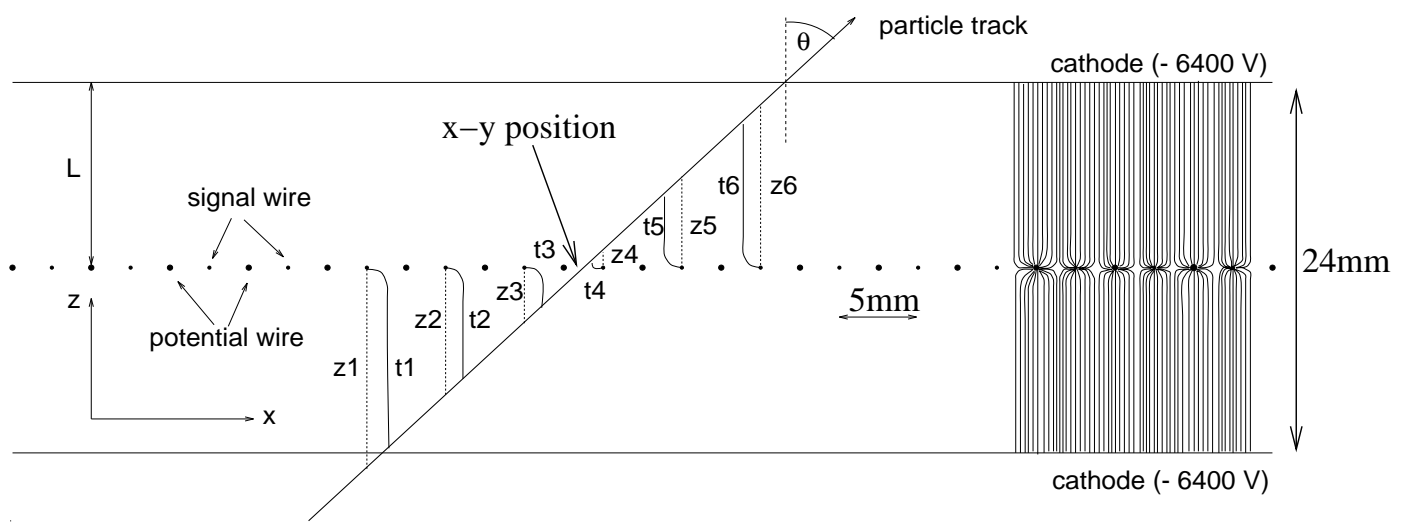

Figure 4.8: Diagram of a VDC layer, showing how different timing signals from each wire can allow the track to be recreated. The reconstructed $\mathrm{x}-\mathrm{y}$ position is also shown.

\section{Scintillation Detectors}

The trigger planes are each comprised of 15 bars of plastic scintillator. The first plane is $3 \mathrm{~mm}$ thick NE 102A, while the second is $10 \mathrm{~mm}$ thick NE Pilot U. Photomultiplier tubes, placed at each end of a scintillator bar, collect the scintillation light via plastic light guides. The second layer gives precise timing of a particle hit in the focal-plane detection system and for this experiment provides the start signal for the neutron polarimeter TDCs. The relative scintillation intensity in each plane (indicative of the relative charged particle energy loss), is characteristic of the incident particle and is used to aid particle identification.

\section{Threshold Gas Cerenkov Detectors}

Particles incident on the Cerenkov detectors pass through a $6 \mathrm{~m}^{3}$ volume of Freon $114\left(\mathrm{CF}_{2} \mathrm{Cl}\right)_{2}$ at atmospheric pressure. Electrons with an energy greater than 10 $\mathrm{MeV}$ will radiate Cerenkov photons in this gas, while charged pions would need an energy of at least $2.7 \mathrm{GeV}$ to produce Cerenkov photons. These photons are then reflected by $2 \times 6$ spherical mirrors, each of area $40 \times 50 \mathrm{~cm}$, which are positioned at the top of the spectrometer facing oncoming particles. The reflected light is then caught by 2 rows of 6 light collection funnels $(18.4 \mathrm{~cm}$ diameter), which are mounted on each side of the spectrometer in front of 5 " photomultiplier tubes. A coincident signal then in these photomultipliers filters out the negatively charged pions, which do not produce Cerenkov light at the energies we are operating at, 
from the electron events with very high efficiency (99.98\%).

\section{Calibration}

The purpose of the magnetic spectrometer is to reconstruct the position and momentum of the scattered electron at the reaction vertex which in the target reference frame is $\left(p_{T}, \theta_{T}, \phi_{T}, x_{T}, y_{T}, z_{T}\right)$. The spectrometer tracking detectors (VDCs) measure the position and direction of the electron after it has traversed the magnetic fields in the focal-plane frame $\left(x_{F}, y_{F}, z_{F}, \theta_{F}, \phi_{F}, \delta_{F}\right)$. This experiment mainly requires the scattered electron momentum $\left(p_{T}, \theta_{T}, \phi_{T}\right)$ which to first order is related to the focal-plane coordinates by $p_{T} \rightarrow x_{F}, \theta_{T} \rightarrow y_{F}$. However to transform accurately back to the target frame requires a knowledge of the inverse transfer matrix, (TMA).

In principle, elements of the TMA can be calculated directly from the known magnetic optics, but in practice these complex calculations are largely avoided by calibrating from well understood experimental data. Various values for $p_{T}$ can be selected by taking data with different beam energies while keeping the spectrometer at a fixed angle. $\theta_{T}$ and $\phi_{T}$ can be selected by using a sieve collimator in front of the first magnet, each hole in the sieve corresponding to a known $\left(\theta_{T}, \phi_{T}\right)$ bin and $y_{T}$ can be varied by using several separated sheets of target material. In this way the TMA may be mapped empirically.

\subsection{Measuring the Neutron Polarisation}

The neutron polarimeter relies on the spin-orbit dependence $\left(V_{s o} l \cdot s\right)$ of the nucleon-nucleon (or nucleus) interaction to produce an asymmetry in the azimuthal distribution of a scattered neutron. This asymmetry is directly related to the polarisation of the neutron and is used to determine the ratio of polarisation components $\frac{P_{x}^{n}}{P_{z}^{n}}$, which is required for the measurement of $G_{E}^{n}$, (sec. 3.1.2).

\subsubsection{Polarised Nucleon-Nucleon Scattering}

Nucleon-nucleon scattering is in itself a very productive branch of nuclear physics, being the major source of experimental input for determining semi-phenomenological "realistic" nucleon-nucleon potentials. The density matrix formalism was introduced by Wolfenstein and Ashkin to describe nucleon-nucleon scattering and is 


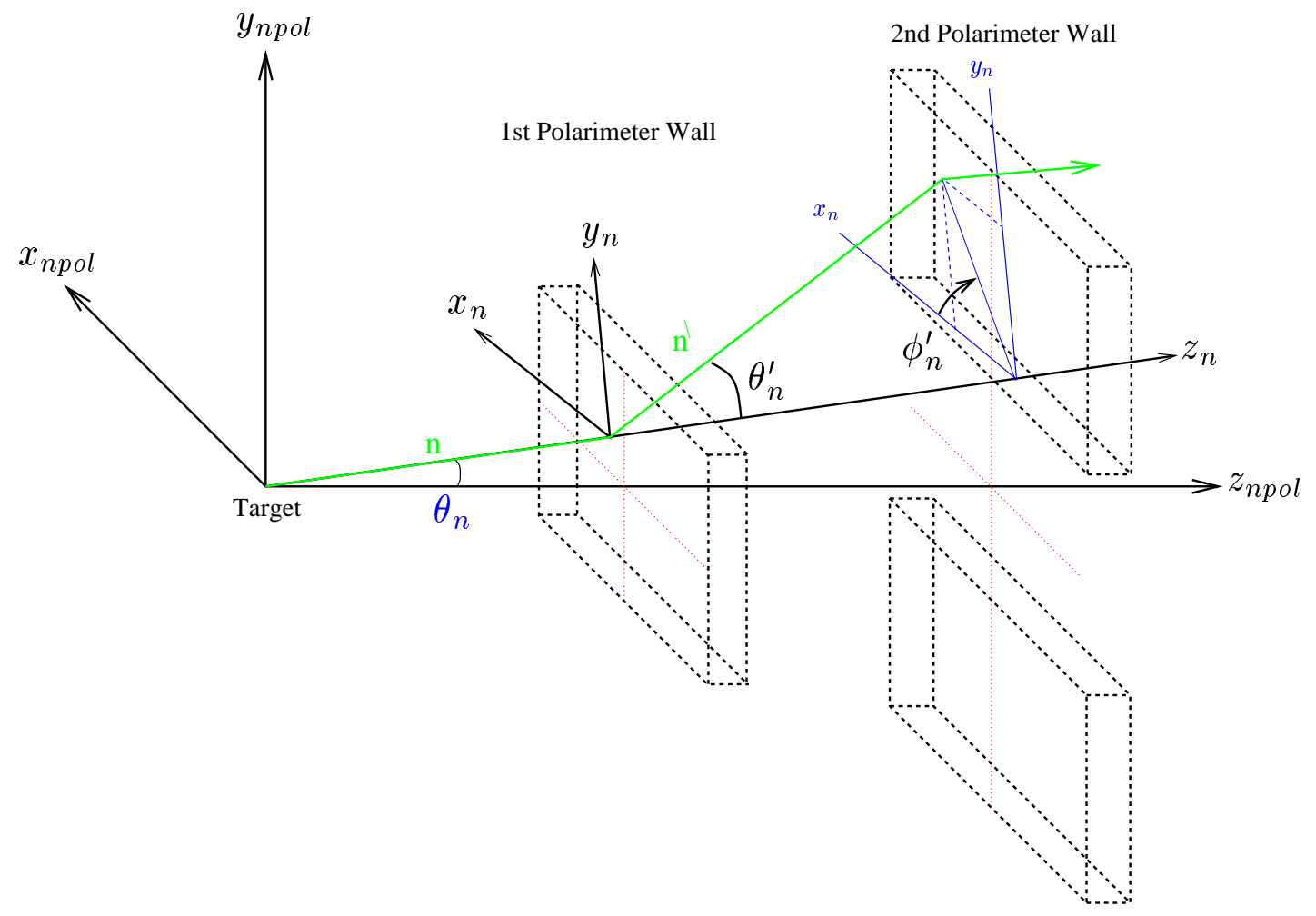

Figure 4.9: Neutron scattering in the first hydrocarbon scintillator wall of the polarimeter. The npol coordinate system has $z_{\text {npol }}$ through the centre of the first wall, $y_{n p o l}$ is vertical and $x_{n p o l}$ makes a right-handed coordinate system. The incident neutron coordinate system $\left(x_{n}, y_{n}, z_{n}\right)$ is then a rotation giving the $z_{n}$ axis along the neutron momentum as in figure 5.23. 
the basis for the subsequent discussion which relates the azimuthal asymmetry to the incident neutron polarisation [56].

If a fraction of a beam of particles is in a pure quantum state the beam expectation value of an operator $\mathrm{A}$ is

$$
\langle A\rangle=\operatorname{Tr}(\rho A)
$$

Where $\rho$ is introduced as the density matrix and $\operatorname{Tr}(M)$ refers to the trace of a matrix M. The differential cross section for the scattered nucleon with initial and final momentum $\underline{p}_{n}$ and $\underline{p}_{n}^{\prime}$ respectively, can then be expressed as:

$$
\frac{d \sigma}{d \Omega} \equiv I(\theta, \phi)=\frac{\operatorname{Tr}\left(\rho_{s c}\right)}{\operatorname{Tr}\left(\rho_{i}\right)}=\frac{\operatorname{Tr}\left(M\left(\underline{p}_{n}, \underline{p}_{n}^{\prime}\right) \rho_{i} M^{*}\left(\underline{p}_{n}, \underline{p}_{n}^{\prime}\right)\right)}{\operatorname{Tr}\left(\rho_{i}\right)}
$$

Where $\rho_{i}$ and $\rho_{s c}$ are the incident and final-state density matrices and $M\left(\underline{p}_{n}, \underline{p}_{n}^{\prime}\right)$ is the spin matrix whose elements are the scattering amplitudes in various final spin states for fixed initial spin states. Equation 4.2 is just the standard procedure of summing over the final states and averaging over the initial states. For the case of a polarised incident nucleon beam and unpolarised target, the spin matrix can be described by a two-by-two matrix of the form [57]:

$$
M=a+b \underline{s} \cdot \underline{N}
$$

where $\underline{s}$ is the incident nucleon spin vector and $\underline{N}$ a unit vector orthogonal to the direction $\underline{p_{n}} \times \underline{p_{n}^{\prime}}$, i.e perpendicular to the scattering plane ${ }^{1}$.

Substituting this into equation 4.2 gives a differential cross section of the form,

$$
I=I_{\circ}[1+\alpha \underline{N} \cdot \underline{s}]
$$

where $I_{\circ}=\left|a^{2}\right|+\left|b^{2}\right|$ is the unpolarised cross section and $\alpha(\theta)=2 \operatorname{Re}\left[a b^{*}\right]$ is defined as the analysing power of the reaction; both can be calculated from phase-shift analysis and examples are shown in figure 4.10. The deviation from azimuthal symmetry is then given by the term $\alpha \underline{N} \cdot \underline{s}$, which is a product of the analysing power and transverse polarisation.

The azimuthal angular distribution of the neutrons scattered in the first wall of the polarimeter can therefore be described by [59],

\footnotetext{
${ }^{1}$ For the coordinate systems defined in figure $4.9, \underline{N}=\left(\sin \phi_{n}^{\prime}, \cos \phi_{n}^{\prime}, 0\right)$.
} 

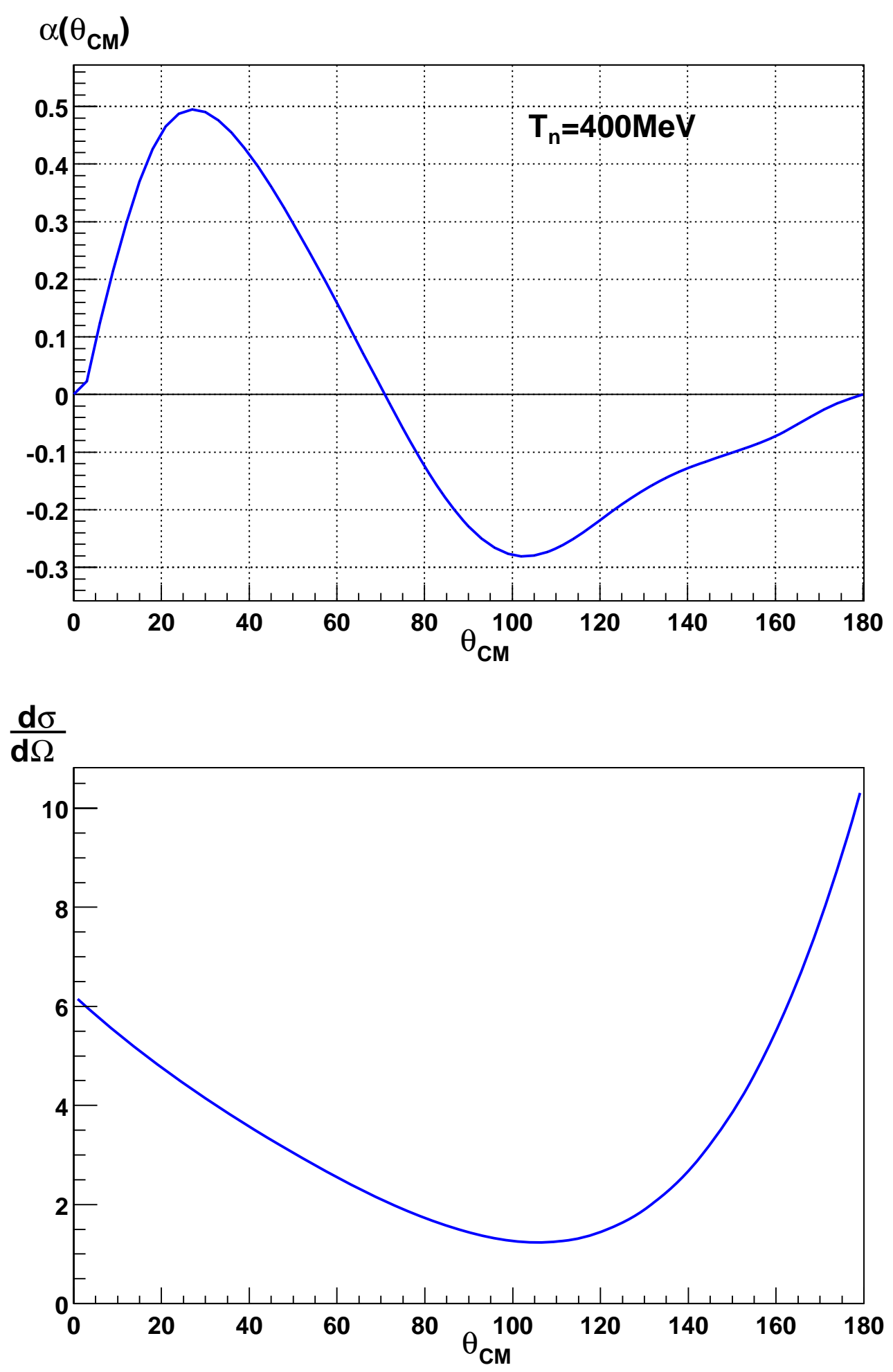

Figure 4.10: The analysing power (top) and differential cross-section (bottom) for $n p$ scattering, from SAID [58] partial-wave analysis. 


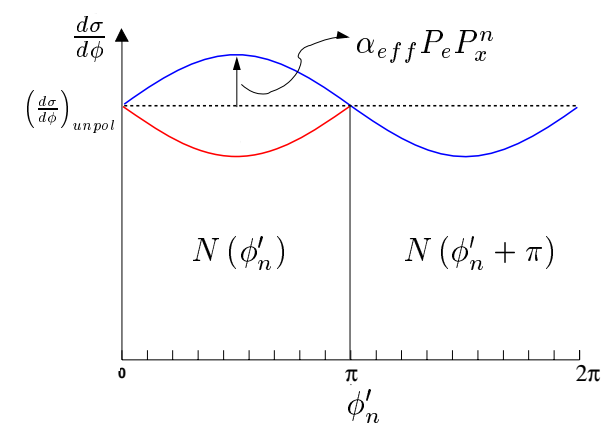

Figure 4.11: Idealised $\phi_{n}^{\prime}$ distribution showing the "up-down" asymmetry (blue line).

$$
\sigma\left(\theta_{n}^{\prime}, \phi_{n}^{\prime}\right)=\sigma_{\circ}\left(1+P_{e} \alpha_{e f f}\left[P_{x}^{n} \sin \phi_{n}^{\prime}+P_{y}^{n} \cos \phi_{n}^{\prime}\right]\right)
$$

when the neutron polarisation is written with the beam polarisation factored out, i.e. $P_{n} \rightarrow P_{e} P_{n}$. $T_{n}$ is the incoming energy of the neutron, $\theta_{n}^{\prime}$ and $\phi_{n}^{\prime}$ are the neutron scattering angles in the first wall, measured with respect to the incident neutron and $P_{x}^{n}, P_{y}^{n}$ are the transverse components of the neutron polarisation in the coordinate system of the incoming neutron (fig. 4.9). $P_{e}$ is the polarisation of the electron beam, $\sigma_{\circ}$ is the polarisation independent cross section and $\alpha_{e f f}$ is the effective analysing power of the first wall. This is the analysing power $\alpha\left(T_{n}, \theta_{n}^{\prime}\right)$ averaged over the angular acceptance of the polarimeter. Thus by measuring the azimuthal distributions $N\left(\phi_{n}^{\prime}\right)$, of neutrons scattered in the first wall, we may determine the transverse $(\mathrm{x}, \mathrm{y})$ components of the incident neutron polarisation when $\alpha_{\text {eff }}$ is known. If $P_{y}^{n} \simeq 0$ (sec. 3.1.2), the azimuthal distribution is sinusoidal (fig. 4.11), so we can construct an asymmetry $A$, that is proportional to $P_{x}^{n} 2$ :

$$
A=P_{e} \alpha_{e f f}\left(T_{n} \theta_{n}^{\prime}\right) P_{x}^{n}=\frac{N\left(\phi_{n}^{\prime}\right)-N\left(\phi_{n}^{\prime}+\pi\right)}{N\left(\phi_{n}^{\prime}\right)+N\left(\phi_{n}^{\prime}+\pi\right)}
$$

Determining $P_{x}^{n}$ in this manner obviously requires an accurate knowledge of the electron beam polarisation and the effective analysing power of the neutron polarimeter.

\footnotetext{
${ }^{2} N\left(\phi_{n}^{\prime}\right)$ is the number of neutrons with $0<\phi_{n}^{\prime}<\pi$ and $N\left(\phi_{n}^{\prime}+\pi\right)$ the number with $\pi<\phi_{n}^{\prime}<2 \pi$.
} 


\subsubsection{Spin Precession Technique}

Although the electron polarisation may be measured using Møller polarimetry, determining the effective analysing power of the neutron polarimeter is not so straightforward. In principal $\alpha_{\text {eff }}$ could be calculated as the analysing power $\alpha_{H}(\theta)$ is well known for $p\left(\vec{n}, n^{\prime}\right) p$. But the scintillator contains carbon as well, $\alpha_{C}(\theta)$ for ${ }^{12} C\left(\vec{n}, n^{\prime}\right) X$ is unknown and not kinematically separable from neutron-hydrogen scattering in the scintillator. It could be measured using a neutron beam of known energy and polarisation, but even if the polarimeter was calibrated at a neutron beam facility, the results would need to be corrected for the effects of the quite different background radiations at the Mainz electron beam facility. In addition we also require the longitudinal neutron polarisation $\left(P_{z}^{n}\right)^{3}$, to determine $G_{E}^{n}$. Previous Mainz $D\left(\vec{e}, e^{\prime} \vec{n}\right) p$ experiments [20, 37], overcame these factors by using a spin precession technique first suggested in [38] and this same technique has subsequently been used for this experiment.

For a neutron flight path L, through the field of the magnet, the spin will precess through an angle of $\chi$ :

$$
\chi=\frac{2 \mu_{n}}{\hbar c} \frac{1}{\beta_{n}} \int_{L} B d l
$$

where $\mathrm{B}$ is the magnetic field and $\beta_{n}$ the velocity of the neutron in units of $c$.

If the neutron spin is precessed so that $P_{x}^{n}=0$ then the asymmetry in equation 4.4 will vanish $(l \cdot s=0$ as the spin is in the direction of the neutron momentum). At this point the precession angle will give the ratio of the transverse and longitudinal components of the polarisation via a simple trigonometric relation:

$$
\tan \chi_{\circ}=\frac{P_{x}^{n}}{P_{z}^{n}}
$$

Measuring the asymmetry (eqn. 4.4) as a function of magnetic field strength, allows the determination of this angle of zero crossing. Thus the ratio $\frac{P_{x}^{n}}{P_{z}^{n}}$ is obtained independent of the effective analysing power of the polarimeter. It should be noted however that although $\alpha_{e f f}$ does not effect the value of $G_{E}^{n}$, it is advantageous to maximise the analysing power in order to minimise the statistical uncertainty. The variation of $\alpha_{\text {eff }}$ under different kinematics cuts applied to the data is discussed in section 6.3.

\footnotetext{
${ }^{3}$ The $n$ refers to the frame in which the polarisation is defined. (fig. 2.5).
} 


\subsubsection{Helicity Flipping}

Large systematic errors can be caused by any misalignment of the polarimeter and any difference in detection efficiency of the upper and lower second-wall frames. Both of these would lead to false asymmetries due to erroneous azimuthal distributions. To cancel these systematic effects the helicity of the electron beam is flipped, which has the effect of flipping the neutron polarisation and thus the sinusoidal azimuthal dependence of the differential cross section (eqn. 4.3). This is equivalent to transforming $\phi_{n}^{\prime} \rightarrow \phi_{n}^{\prime}+\pi$, i.e. the upper and lower frames switch, thus cancelling out these systematic effects. The asymmetry is now constructed as:

$$
A=\alpha_{e f f}\left(T_{n}, \theta_{n}^{\prime}\right) P_{e} P_{x}^{n}=\frac{\sqrt{N^{+}\left(\phi_{n}^{\prime}\right) N^{-}\left(\phi_{n}^{\prime}+\pi\right)}-\sqrt{N^{+}\left(\phi_{n}^{\prime}+\pi\right) N^{-}\left(\phi_{n}^{\prime}\right)}}{\sqrt{N^{+}\left(\phi_{n}^{\prime}\right) N^{-}\left(\phi_{n}^{\prime}+\pi\right)}+\sqrt{N^{+}\left(\phi_{n}^{\prime}+\pi\right) N^{-}\left(\phi_{n}^{\prime}\right)}}
$$

where $N^{ \pm}$implies the distribution when the helicity is \pm 1 .

\subsubsection{Isospin Symmetry}

Neutron scattering from a nucleon target can be described by the exchange of mesons with either positive or neutral charge, with the former resulting in the nucleons swapping type in the final state. For exact isospin symmetry the probability of exchanging a charged or uncharged meson is the same and the isospin of the nucleons in the final state should not effect the kinematics, thus the analysing power and asymmetry, should not change, regardless of the type of nucleon detected in the second wall.

In fact there is a slight breaking of isospin symmetry as seen in the different masses of the proton and neutron. This also results in the forward-backward asymmetry in the scattering cross section and the deviation of the analysing power from pure sinusoidal dependence (fig. 4.10). Despite the lower cross section and analysing power for forward scattered protons which are incident on the second wall (backward scattered neutrons), the high detection efficiency of protons in the second wall ( factor 4 greater than for neutrons) makes it worthwhile to analyse both forward angle neutrons and protons when detected in the second wall. 


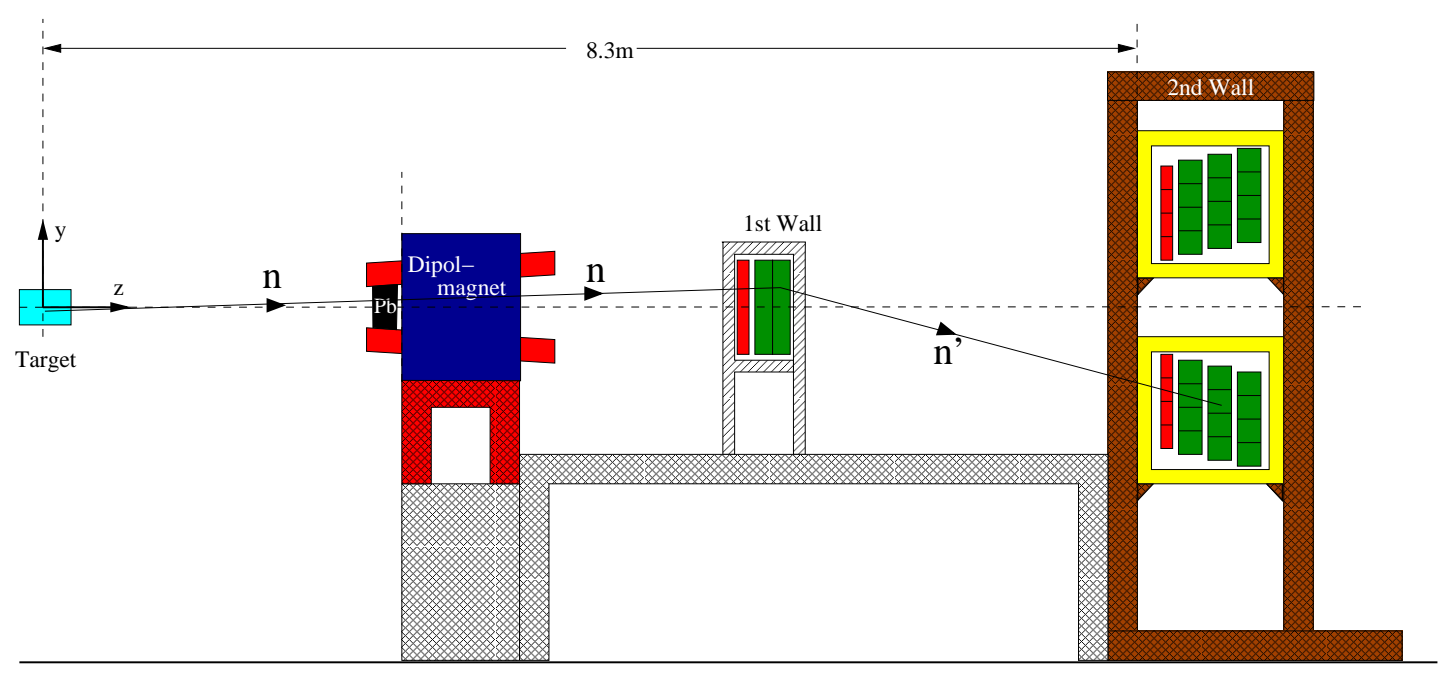

Figure 4.12: The A1 neutron polarimeter. (Not to scale).

\subsection{Neutron Polarimeter}

The polarimeter geometry is based on a design by Taddeucci et al [59] and is shown in figure 4.12. The essential components of a polarimeter for measurement of transverse neutron polarisation are two planes of scintillator orientated perpendicular to the incident neutron direction. The first plane acts as an active analyser using neutron scattering off hydrogen and carbon nuclei and also allows a determination of the incident neutron energy through time of flight measurement. The scattered neutrons are then detected in the second scintillator plane. This allows a reconstruction of the neutron scattering angles $\theta_{n}^{\prime}$ and $\phi_{n}^{\prime}$, where the $\phi_{n}^{\prime}$ distribution is sensitive to the transverse polarisation of the incident neutron (sec. 4.5).

For the polarimeter used in our experiments the first wall consisted of 2 layers of 15 scintillator bars with dimensions $80 \times 7.5 \times 5.0 \mathrm{~cm}^{3}$, orientated vertically. In addition, a layer of $15,1 \mathrm{~cm}$ thick, plastic scintillators, the veto layer, were positioned directly in front of the main wall, to allow the suppression of incident charged particles and make neutron identification possible.

The second wall was split into an upper and lower frame, each of which had 3 layers of $4,180 \times 20 \times 10^{3}$ scintillators stacked horizontally. Again a $1 \mathrm{~cm}$ thick veto bar shadowed each row of second wall bars. An important reason for splitting the 2 nd wall was to avoid the region around the $\left(e, e^{\prime} n\right)$ reaction plane where there is a high background intensity from atomic interactions of the 
electron beam with the target. The upper and lower sections were separated by approximately a metre.

An additional component to the polarimeter design of [59], first suggested by Arnold, Carson and Cross [38] is a dipole magnet with a vertical field. This was used to precess the neutron spin, allowing determination of the longitudinal as well as the transverse spin components (sec. 4.5).

The magnet, the first scintillator plane and the second were positioned respectively $3 \mathrm{~m}, 6 \mathrm{~m}$ and $9 \mathrm{~m}$ from the target. This positioning allowed the two frames of the second wall to be shielded from the target by the dipole magnet, the gap between its pole faces being a window for the 1st wall. All neutrons detected in the 2 nd wall therefore should have come from scattering in the 1st wall, which is another reason for the splitting of the second plane.

Extra shielding was employed to reduce the counting rate of the electromagnetic background in the polarimeter. A $5 \mathrm{~cm}$ thick lead wall was constructed in the gap of the spin-precession magnet to attenuate hard photons and electrons and $5 \mathrm{~mm}$ of aluminium was placed directly in front of each of the scintillator walls to attenuate soft electrons.

Each scintillator bar had a light guide and photo-multiplier tube (PMT) attached at each end. The scintillation light caused by charged particle interactions produced a signal in the PMT's which was then recorded by time-to-digital (TDC) and charge-to-digital (QDC) converters. The QDC's gave information on the energy deposited in a bar. The mean time of the 2 TDC signals was used to find the time of flight of the neutron from the target, while the TDC difference gives the hit position along the length of the scintillator. The other 2 spatial coordinates are given by the position of the hit bar.

\subsection{Data Acquisition}

The trigger electronics and data acquisition system AQUA [60], were responsible for ensuring neutron and electron signals were recorded in the data stream for each event, as efficiently as possible. Front-end VME computers were attached to Spectrometer A and the neutron polarimeter. These read out fastbus ADCs and rate counters (scalers). They then passed the data to an event-builder which merged and synchronised events, after which the data could be formatted and written to an output file. 


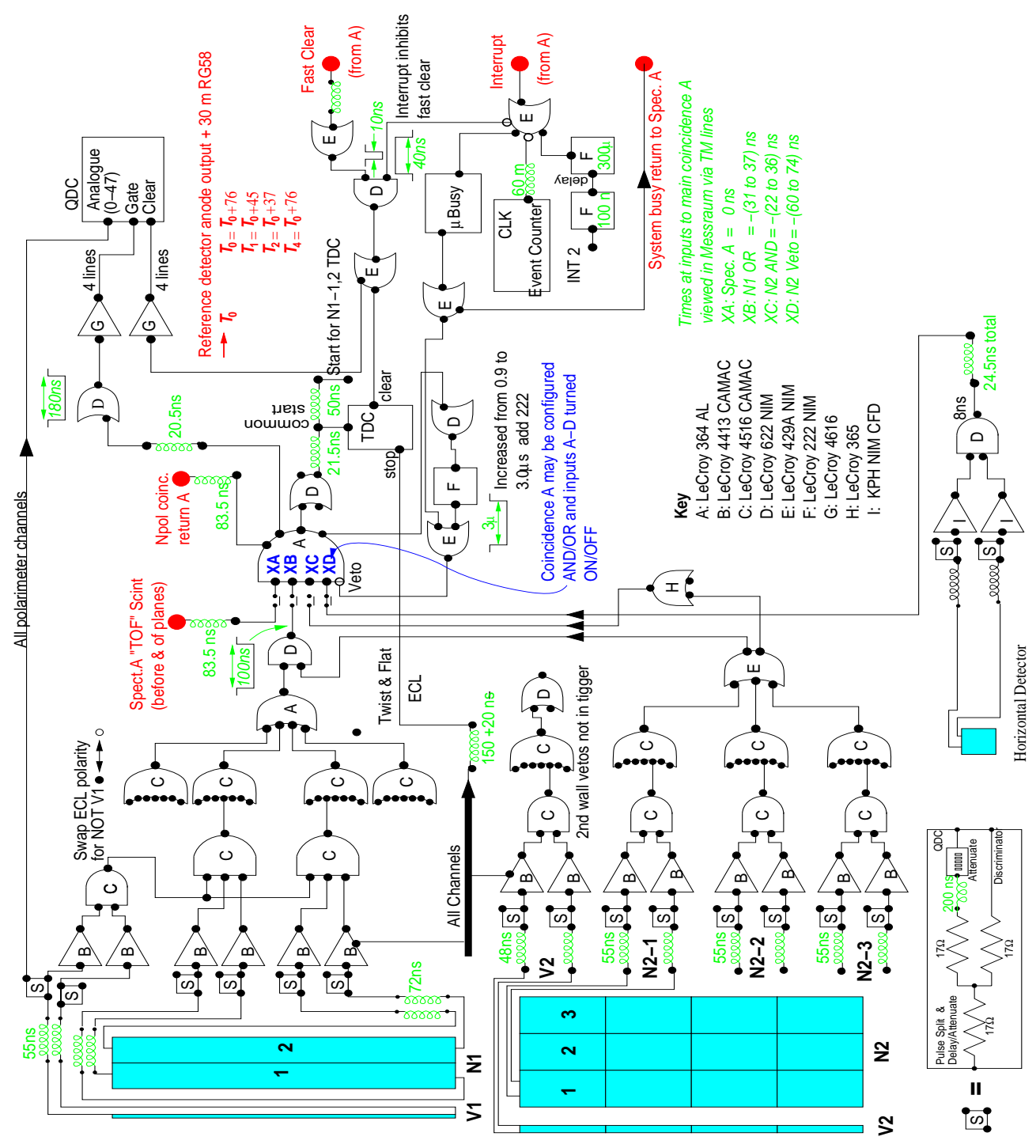

Figure 4.13: The trigger electronics for the neutron polarimeter. 


\section{Electronic Trigger}

The trigger (fig. 4.13) had to discriminate against background signals which otherwise would flood the data acquisition and result in a high dead-time ${ }^{4}$ with many missed true events. The neutron polarimeter signal rate (mostly background) was some orders of magnitude higher than that for Spectrometer A. This is due to the large volume of the polarimeter scintillators and the spectrometer detectors being shielded from direct view of the target and the exit beam-line. The spectrometer focal plane detectors had a relatively "clean" signal, seeing only momentum-analysed particles and cosmic rays.

The central part of the trigger demanded a two-way coincidence (module A in fig. 4.13) between spectrometer $\mathrm{A}$ and the polarimeter. The spectrometer signal $($ Spect $A \rightarrow X A)$ came from an $\mathrm{OK}$ of the second thicker scintillator plane and required a coincidence between the two attached photomultipliers.

For the polarimeter $(N p o l \rightarrow X B)$, signals from the photomultipliers were split (splitterbox S), with one output sent to the ADC's and the other to a discriminator (B). For signals above a predefined threshold (to suppress the high rate of low energy signals), the discriminator output a 50ns or 100ns wide logic signal for the first or second wall respectively. In the case of the second wall an AND was demanded between the two ends of a bar, by a coincidence module (C). The $\mathrm{Npol}_{2}$ signal is then produced by an OR (C then E) of all the second wall bars. $N$ pol $_{1}$ is similar but also requires a NOT signal from the veto detector directly in front of a scintillator bar, for the suppression of charged particles, along with the AND of each end of the bar. An AND (D) of $N p o l_{1}$ and $N p o l_{2}$ was then used to produce a 100ns pulse for the polarimeter signal, $(N p o l \rightarrow X B)$

If there was an overlapping SpectA-Npol signal the coincidence module sent out four signals, (i-iv):

(i) The signal to "Npol coinc. return A" started the VME frontends reading the ADC and TDC modules for the data acquisition. An interupt or fast-clear signal was then sent back to the trigger. The interupt set the $\mu$ Busy module to inhibit further triggers and stop AQUA reading more events until it is finished with the event it is on. When AQUA is finished reading all the modules it resets the $\mu$ Busy allowing new events to be read. The interupt also incremented the event-counter which allowed the event-builder to synchronise events from the

\footnotetext{
${ }^{4}$ Dead-time is the time when the frontend computers are reading the signals in the electronic modules and no new trigger can be sent.
} 
different detectors. The fast-clear reset the ADC and TDC modules a $\mu s$ after the gate start.

(ii)A $180 \mathrm{~ns}$ gate was sent to the polarimeter ADCs to specify the charge integration time. The charge comes from the splitterbox signal which will be proportional to the signal from the photomultipliers and thus the energy deposited in the scintillator bars.

(iii) A common start derived from the $($ Spect $A \rightarrow X A)$ signal, caused by relativistic electrons, was sent to the TDCs. The stops came from the discriminator signals for the individual photomultipliers, so after calibration the difference in these times was related to the time of flight of the neutron (sec. 5.2.5).

(iv)The last signal was used to temporarily disable the Spect.A-Npol coincidence module stopping multiple "Npol coinc. return A" signals.

In addition scaler modules were used to count the number of signals over the discriminator threshold from each photomultiplier. This acts as a monitor of the rates in the detectors which for the front wall elements were $>10^{6} \mathrm{~Hz}$ in the worst cases.

\section{AQUA}

The newly revised A1 data acquisition system, AQUA was responsible for reading the signal digitisation modules, event-building and writing the data to disc. After a valid event trigger each of the VME front-end computers, which were positioned in the experimental hall next to their detectors, read the TDC and ADC modules, the event-counter and scalers via an interface to fastbus and sent the data to the event-builder in the counting room. The event-counter information then allowed the data streams from each detector to be merged by the event-builder. In addition to the detector data, control data e.g. high voltages, target temperature, was also merged into the data stream. This setup allowed an interupt rate of $200 \mathrm{~Hz}$ for the experimental data taking, so we could take advantage of $10 \mu \mathrm{A}$ beam currents.

The data were then written to the output run files. For storage purposes the file size was taken to be $\sim 100 \mathrm{Mb}$ which for $10 \mu \mathrm{A}$ beam currents corresponded to around 30 minute "runs". 


\section{Chapter 5}

\section{Analysis}

\subsection{Simulation of the Neutron Polarimeter}

Prior to undertaking the data-analysis there was an uncertainty as to the physics processes occuring in the analysing scattering reaction in the first wall of the neutron polarimeter. Previous recoil polarisation anlaysis had assumed the analysing power came predominantly from the neutron scattering on hydrogen nuclei, hence cuts were applyed to maximise neutron-hydrogen scattering over scattering interactions with the carbon [61]. It was also unclear if the asymmetry of protons detected in the second wall would give a similar asymmetry as neutrons (i.e. isospin symmetry sec. 4.5.4). Clarification of these issues was clearly important before a detailed analysis of the data.

In order to understand better the response of the polarimeter a Monte Carlo computer model was written, based on the CERN GEANT4 (G4) Monte Carlo library [62], with analysis of the MC output performed using the ROOT data analysis libraries [63]. The four main parts to the simulation are polarimeter geometry, event generation, particle interactions and data analysis. These are described in the following, where named components of the G4 software system are given in bold face. G4 is an object-orientated system using the $\mathrm{C}++$ language where code is based on the concept of class [64].

\subsubsection{Polarimeter Geometry}

To simulate the physical experiment, G4 requires the detector geometry to be defined via the G4Detector Construction class. A variety of standard geometry classes were used to define the volume of the various polarimeter components. 


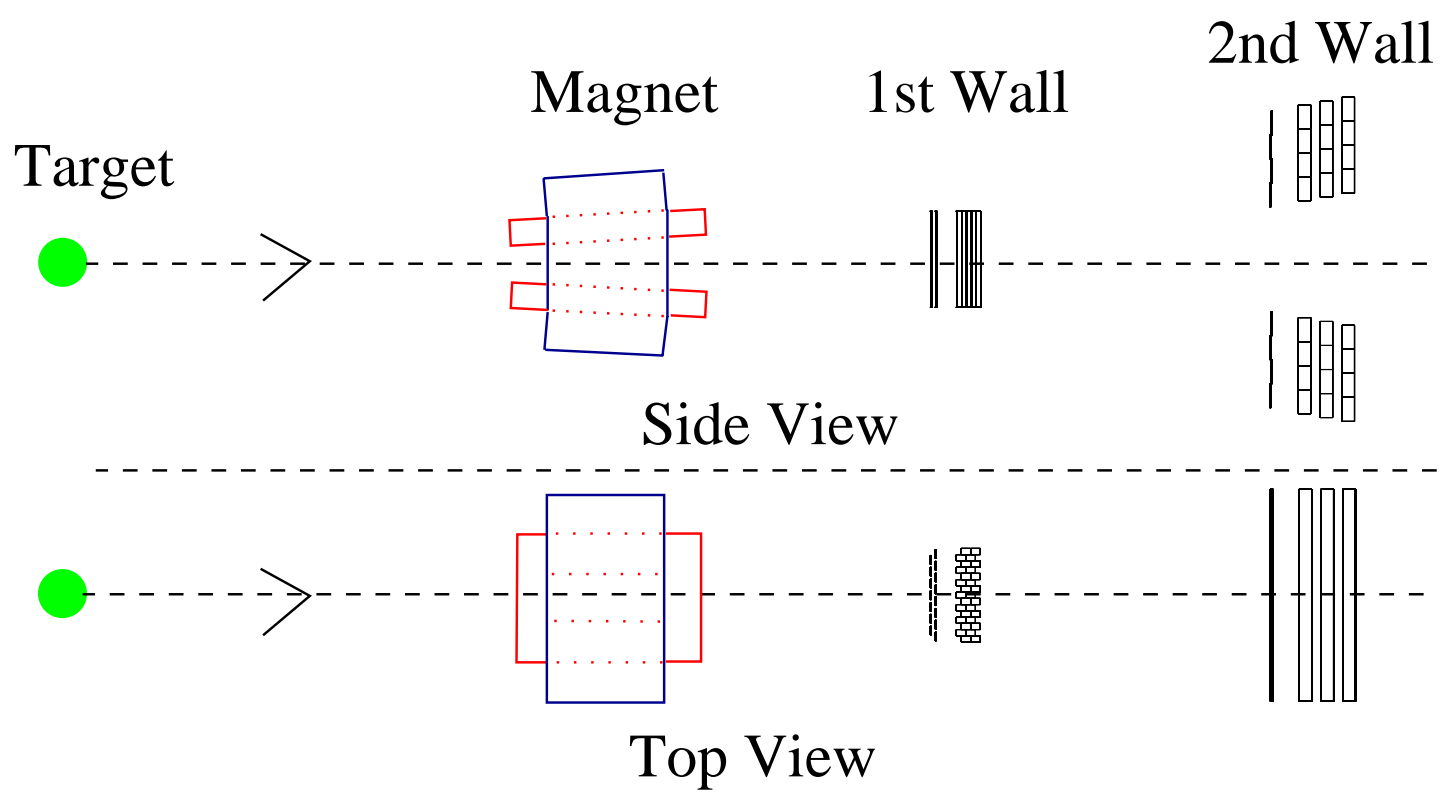

Figure 5.1: Scale layout of the polarimeter used in the GEANT4 simulation.

Included for the simulation were the scintillator arrays constituting the front and rear walls of the polarimeter and the spin precession magnet. The scintillator material was taken as a composition of 10 atoms of hydrogen and 9 atoms of carbon with a density of $1.032 \mathrm{gcm}^{-3}$. Figure 5.1 shows a visualisation of the simulation geometry. The bars were coded as G4SensitiveDetector, to allow particle interactions occurring inside their volume to be recorded, with sufficient flexibility to allow easy changing of the polarimeter positions, necessary for different $Q^{2}$ settings.

\subsubsection{Event Generation}

Generating an event is started by selecting an initial momentum for the neutron for the G4ParticleGun object, which "fires" the neutron at the polarimeter. As we were interested only in events where the electron scatters into the spectrometer we randomly selected the scattered electron momentum, $\vec{P}_{e^{-}}^{\text {spec }}$ from angular and momentum distributions obtained in the real experiment, as shown in figure 5.2. The incident electron was given by the beam direction and energy. These quantities define the reaction $Q^{2}$. The corresponding virtual photon will have momentum given by equation 5.1, which in the experiment has a direction cen- 

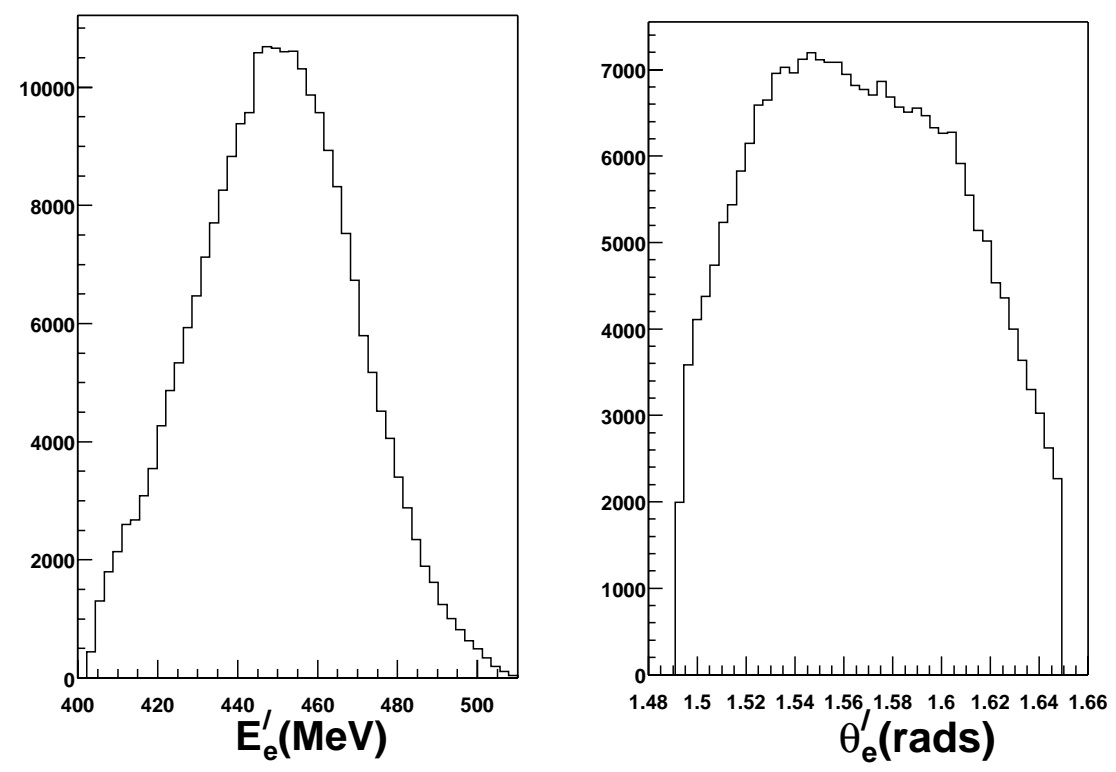

Figure 5.2: Scattered electron energy and angle, reconstructed from Spectrometer A.

tered on the polarimeter ${ }^{1}$. The electron is also given, at random, a longitudinal polarisation direction (beam helicity), of \pm 1 .

$$
\vec{P}_{\gamma}=\vec{P}_{e^{-}}^{\text {beam }}-\vec{P}_{e^{-}}^{\text {spec }}
$$

As the neutron "target" was in reality deuterium the neutron will have a Fermi motion exactly opposite to that of the proton in the deuteron. The neutron was therefore given a momentum $\vec{P}_{n}^{\text {fermi }}$ selected randomly from the distribution obtained from [65]. The resulting momentum of the neutron incident on the polarimeter was then given by :

$$
\vec{P}_{n}=\vec{P}_{\gamma}+\vec{P}_{n}^{\text {fermi }}
$$

The G4PrimaryGeneratorAction procedure was called at the start of each event and was responsible for calculating the initial neutron momentum in this way and assigning it to the G4ParticleGun.

\footnotetext{
${ }^{1}$ As the experimental kinematics were selected this way.
} 


\subsubsection{Particle Interactions}

Once an event had been generated and the neutron had been fired from the G4ParticleGun, the G4SteppingManager looked after the neutron's progress through the polarimeter as a series of G4Steps. For each G4Step the G4SteppingManager decided which process had occurred and then applied the appropriate model to find the outcome of the interaction. The possible processes and models were defined in G4PhysicsList. For the $(\sim 100 \mathrm{MeV})$ neutrons the processes defined were G4HadronElasticProcess and G4HadronInelasticProcess. The default process, if no interaction occurred during the step is G4Transport which simply moved the neutron without deviation from its momentum direction. Investigation of the GEANT4 neutron interaction models showed them to be insufficiently realistic for the energy range of our experiment, so that new models had to be created, these are outlined below. The neutron kinematics were stored by G4TrackingManager as a series of G4Tracks, with each G4Step having a corresponding G4Track.

\section{Process Selection}

At the start of a G4Step the InteractionLength $=\sum_{i} \frac{1}{\sigma_{i}}$ (equivalent to the mean free path) was calculated for all the possible physics processes, where $\sigma_{i}$ is the cross-section for a process occurring on element $\mathbf{i}$ of the compound material (e.g. scintillator or air) that the neutron is currently in. A PhysicalInteractionLength for each process was then determined as :

$$
\text { PhysicalInteractionLength }=\text { InteractionLength } \times[-\log (R)]
$$

Where $\mathrm{R}$ is a random number in the range $[0,1]$. The process which has the shortest PhysicalInteractionLength is then selected for that G4Step. Note, the PhysicalInteractionLength for the G4Transport process (no interaction) is simply the distance to the next volume.

\section{Neutron Scattering Models}

For a neutron scattering in a plastic scintillator there are a number of different possible reaction channels from both the Hydrogen and Carbon nuclei. For this simulation the considered interactions followed the STANTON code of Cecil et al [66]. The interactions were then evaluated by procedures based on STANTON, 
G4 and newly developed scattering models.

The list of interactions considered by STANTON is as follows :

1. $n+p \rightarrow n+p$

2. $n+C^{12} \rightarrow n+C^{12}$ non-diffractive

3. $n+C^{12} \rightarrow n+C^{12}$ diffractive

4. $n+C^{12} \rightarrow n+C^{12}+\gamma$

5. $n+C^{12} \rightarrow \alpha+B e^{9}$

6. $n+C^{12} \rightarrow n+3 \alpha$

7. $n+C^{12} \rightarrow n+p+B^{11}$

8. $n+C^{12} \rightarrow 2 n+C^{11}$

The semi-empirical cross-sections which STANTON uses for these interactions are shown in figure 5.3. It is clear from this figure that reaction channel (7) and (1) are the most important for the present experiment where the neutrons have energies of 300 or $400 \mathrm{MeV}$. Elastic scattering on $C^{12}$, (2), also has a significant cross-section, but the recoiling Carbon deposits too little energy in the scintillator and consequently the polarimeter is insensitive to this channel.

An important quantity in the interaction is the analysing power, which gives the scattering asymmetry. Unfortunately this is well known only for elastic np scattering (fig. 4.10). For the purposes of this simulation it has been assumed that reaction (7) is a quasi free scattering process with an analysing power similar to the elastic np reaction, (1). The other reaction channels are assumed to have zero-analysing power, although it is likely to be non-zero but small.

Reaction channel (1) elastic np scattering was modelled in the following way:

1. The centre of mass four-momentum was calculated as $P_{C M}=\left(E_{C M}, \underline{P}_{C M}\right)=$ $P_{n}+P_{p}$, where $P_{n, p}$ are the four-momenta of the initial neutron and proton.

2. The breakup momentum $P_{N}^{*}$, for either particle in the centre of mass was calculated as $P_{N}^{*}=\sqrt{\frac{\left[\left(E_{C M}^{2}-\left(M_{n}+M_{p}\right)^{2}\right)\left(E_{C M}^{2}-\left(M_{p}-M_{n}\right)^{2}\right)\right]}{2 E_{C M}}}$. 


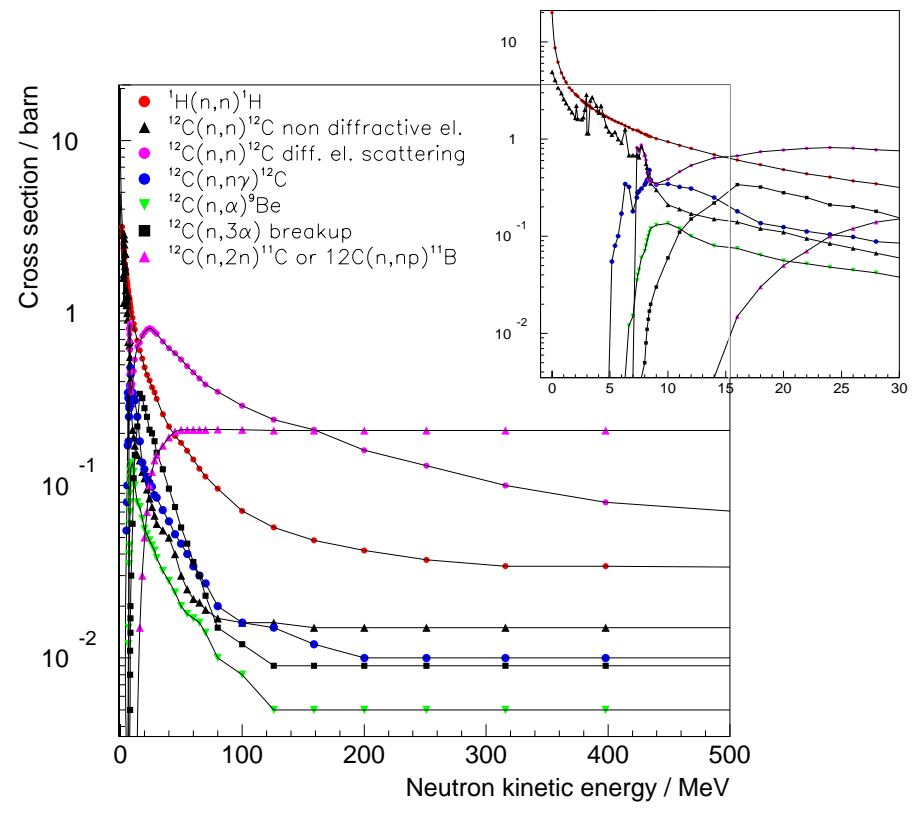

Figure 5.3: Cross sections for neutrons scattering on hydrogen and carbon.

3. $\theta_{C M}$ was chosen using a weighted random number generator based on distributions (fig. 5.4), which were generated using the SAID [58] partial-wave analysis of n-p scattering data.

4. The analysing power was found as a function of $\theta_{C M}$ and $T_{n}$, the kinetic energy of the neutron (fig. 4.10) also from SAID.

5. The azimuthal angle $\phi_{C M}$ was found from a distribution weighted by the analysing power (fig. 4.11).

6. $P_{C M}, \theta_{C M}$ and $\phi_{C M}$ were then combined to give the momentum vector of the neutron in the centre of mass frame. The proton was given the opposite momentum and the two momenta were boosted back into the lab. frame.

Reaction channel (7) quasi-elastic np scattering was modelled in a similar way to reaction channel (1). The neutron scatters off a proton which is "off mass shell", and had an initial Fermi momentum in the carbon nucleus. The recoil, excited Boron nucleus was treated as a spectator and it thus had a momentum opposite to the initial proton fermi momentum due to conservation of momentum. The initial Fermi momentum was taken from distributions derived from harmonic oscillator potentials assuming the proton was in either an s or p-state. Spectral 


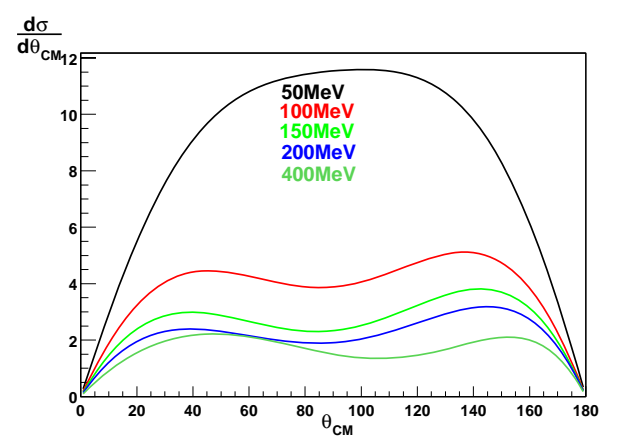

Figure 5.4: The differential cross sections for ${ }^{1} H(n, p)$ used to select the protonneutron centre-of-mass angle.

analysis of nucleon knockout from carbon [67] shows that excitation energy from p-shell knockout is small and for this model it was taken to be zero. For s-shell knockout the excitation energy has a broad distribution and for this simulation it was simply taken to be constant in the range $25-50 \mathrm{MeV}$ zero elsewhere. A summary of the kinematics is shown in Figure 5.5.

\subsubsection{Light Response}

An additional calculation has to be added to the G4 code to simulate the light response of the scintillator. When a proton loses energy through atomic interactions to create scintillation light, the total energy of light detected by the photomultipliers is not linearly related to the amount of energy lost by the proton. This non-linearity is thought to be due to quenching of the scintillation processes, where the ionisation density is high [68], which will occur increasingly at low energies where the proton velocity is small. This is particularly true when the kinetic energy of the proton is only a few $\mathrm{MeV}$. The light response function used is that of Madey et al [69] which was also adopted in [66] for the STANTON code. They fitted their measurements and those of [70] to a semi-empirical function of the form,

$$
T_{e}=a_{1} T_{p}-a_{2}\left[1.0-\exp \left(-a_{3} T_{p}^{a_{4}}\right)\right]
$$

Where the fit parameters were found to be $a_{1,2,3,4}=0.95,8.0,0.1,0.90$ and the light response is given in terms of an equivalent electron light output which is assumed to be linear with energy. Therefore, by converting proton energy $T_{p}$, to equivalent electron light response $T_{e}$, we effectively simulate the response of 


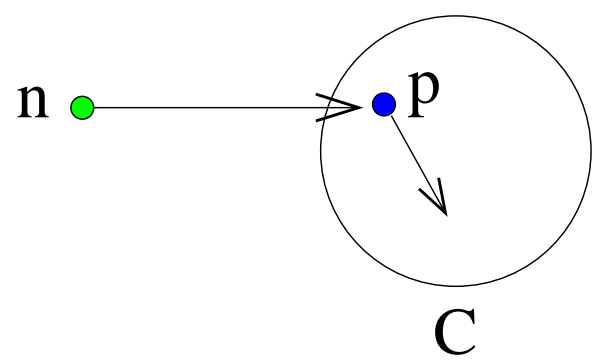

Before

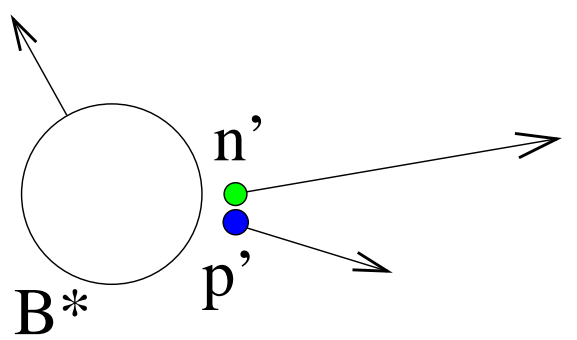

After

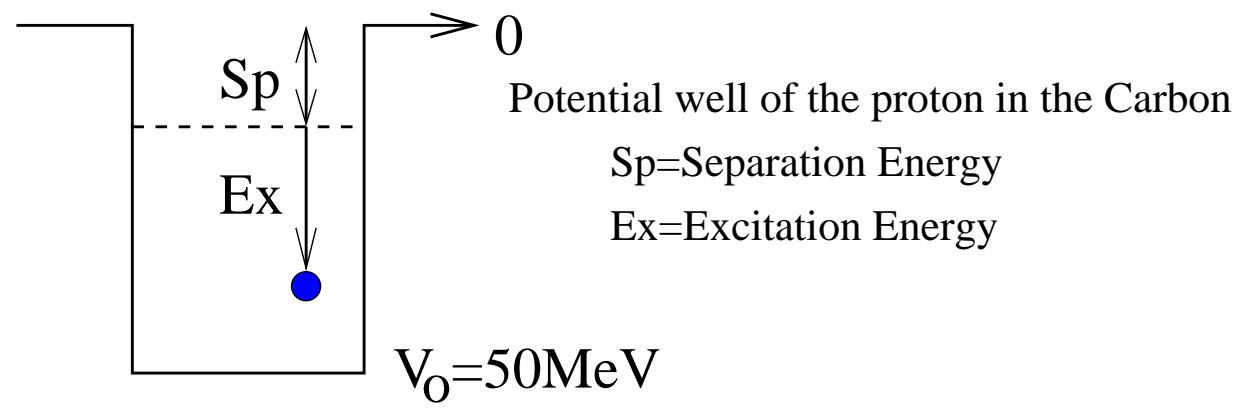

Before : $n\left(M_{n}, E_{n}, \vec{P}_{n}\right), p\left(M_{p}^{o f f}, E_{p}, \vec{P}_{f e r m i}\right), C\left(M_{C}, 0,0\right)$

After : $n^{\prime}\left(M_{n^{\prime}}, E_{n^{\prime}}, \vec{P}_{n^{\prime}}\right), p^{\prime}\left(M_{p^{\prime}}, E_{p^{\prime}}, \vec{P}_{p^{\prime}}\right), B\left(M_{B}^{*}, E_{B},-\vec{P}_{\text {fermi }}\right)$

The proton state is selected with a probablity ratio of 2:4 for s:p states.

Ex is taken as zero for p-state and chosen at random from the potential well depth for s-state. Giving the mass of the excited recoil Boron as :

$M_{B}^{*}=M_{C}-M_{p}-S p-E x$

The initial energy of the proton can then be found from the conservation of energy :

$$
\begin{aligned}
& M_{C}=E_{B}+E_{p}, \text { with } E_{B}=\sqrt{\left(M_{B}^{*}\right)^{2}+P_{\text {fermi }}^{2}} \\
& \Longrightarrow E_{p}=M_{C}-\sqrt{\left(M_{B}^{*}\right)^{2}+P_{\text {fermi }}^{2}}
\end{aligned}
$$

The four vectors of the initial neutron and proton are now known and can be used as in the elastic np scattering model.

Figure 5.5: Quasi-elastic np scattering. 


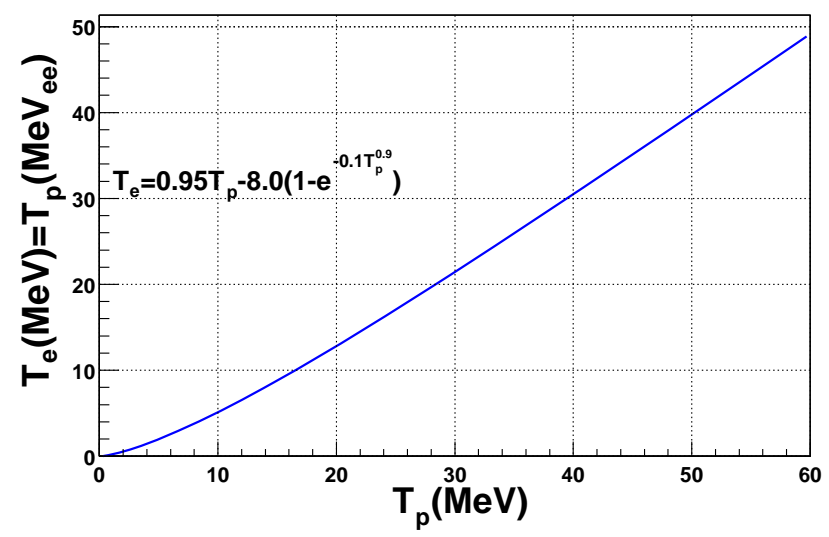

Figure 5.6: Light response function.

the scintillator to the proton energy loss in units of $\mathrm{MeV}_{e e}$, allowing a direct comparison in energy deposited between the simulation and real data when the real data is calibrated in units of $\mathrm{MeV}_{e e}$ (electron equivalent). Equation. 5.2 is plotted in figure 5.6.

\subsubsection{Data Analysis}

If a neutron interaction occurred in the scintillators then the following quantities were stored in a G4Hit object : time(time of flight), energy deposited (in $\mathrm{MeV}$ and $\mathrm{MeV}_{e e}$ ), hit position and bar number. In addition further information about the interaction was stored in a GenEvent object : initial and final neutron four-vectors, reaction channel and number of interactions.

These objects were then analysed after the event had been fully tracked by G4Event- Action, the main part of which mimics the real data analysis, i.e. recreating energies from times of flight, scattering angles from hit positions, checking for veto coincidences and sorting multiple hits, (sec. 5.4.3 for details). The results were then written to ROOT ntuples, as for the real analysis, but with the additional GenEvent information giving details of the interaction physics, which allowed the selection of particular reaction channels when looking at kinematic distributions.

These events were then analysed to determine the effective analysing power of the simulated polarimeter. This was calculated through equation 4.7, where $N^{+}\left(\phi_{n}^{\prime}\right)$ were obtained from the simulated $\phi_{n}^{\prime}$ distributions. The beam polarisation and transverse neutron polarisation were taken to be 1 and so the asymmetry 


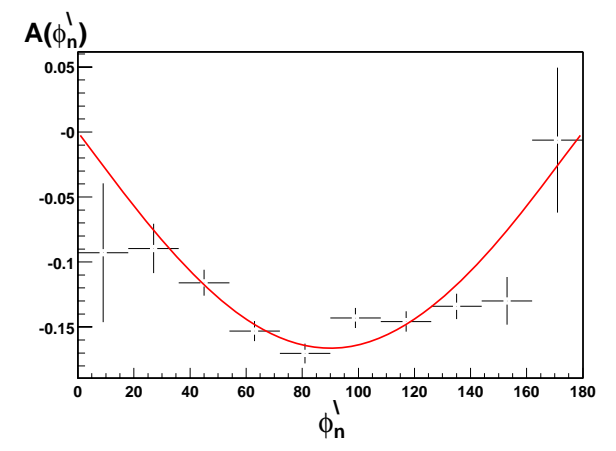

Figure 5.7: Simulated asymmetry (crosses) versus azimuthal scattering angle. The red line curve is a one-paramater(amplitude) sinusoidal fit.

in equation 4.7 is equivalent to the effective analysing power over the acceptance of the polarimeter $\alpha_{\text {eff }}$. A typical plot showing the resulting asymmetry versus $\phi_{n}^{\prime}$ is shown in figure 5.7. The amplitude of the one-parameter sinusoidal fit gives $\alpha_{\text {eff }}=0.166 \pm 0.004$.

\subsection{Neutron Polarimeter Calibrations}

The most important calibration for the neutron polarimeter was the spatial calibration to determine the "hit" positions in each wall, that is the positions where the neutrons interacted with the nuclei in the plastic scintillator. A good calibration of these hit positions allowed an accurate determination of the scattering angles which were used to derive $G_{E}^{n}$. In addition knowledge of the time of flight yields the energy of the neutron before (first wall) and after (second wall) the analysing reaction.

The spatial calibration required the physical positions of the scintillators in the frame of the neutron polarimeter and the differences of the hit times from the photomultipliers attached at each end of a scintillator. The time of flight from the mean hit time of the two photomultipliers required a calibration of the TDC start time derived from the hit time in the trigger plane of the spectrometer. It was therefore important to correct for electronic effects in the TDC signal, in particular since leading-edge discriminators were used a walk correction had to be applied. This also required the amplitude of the photomultiplier signal and is discussed in the following sections.

The calibrations $[71,72]$ were applied to the raw data as part of the COLA ++ analysis procedure (sec. 5.4.1). The run.db (database) input file was used to 
identify the correct calibration file and polarimeter position for a particular run time during the extended data taking period.

\subsubsection{QDC Pedestals}

The QDC pedestal is an offset intrinsic to an individual QDC channel. It is due to a small D.C. current which is applied to the QDC input to ensure the input current is always negative even if there is a positive overshoot on the input signal. Thus the QDC zero point occurs at a non-zero channel which has to be subtracted. A sharp peak was clearly visible at this channel, since most recorded events for a particular common gated QDC are null, making the correction straight forward to determine.

\subsubsection{TDC conversion-gain calibration}

The TDCs (Phillips 10C6), have 1024 channels and the full scale range was set to 100ns implying that each channel should have a width of $\frac{100}{1024} \simeq 0.1 \mathrm{~ns}$. This was checked using a signal from a photomultiplier which was split, one signal being used as a common start for the TDCs the other being put through a delay, stepped in delay lengths of 8ns and then used to stop the TDCs. The delay was checked using an oscilloscope and the TDCs read by the acquisition software AQUA ++ . Comparisons of the delay to the TDC channels showed that the channel width was indeed $0.1 n$ sith an uncertainty of less than $1 \%$.

\subsubsection{Walk Correction}

The start time for the polarimeter TDCs came from the trigger scintillator plane of the electron spectrometer. The delay of this signal is well known with respect to the time of electron scattering in the target, since the electrons are relativistic and will therefore have a constant velocity $(\simeq c)$, to good approximation, with a correction applied offline to account for slight delay differences between elements of the trigger plane. The stop signal comes from the leading edge discriminators attached to the polarimeter PM tubes (fig. 4.13) and as a result is subject to a leading edge walk effect [73]. Due to finite rise time a signal with a high pulse height will cross the discriminator threshold before a signal with a low pulseheight (fig. 5.8) and so a correction must be applied in the offline analysis, to the individual TDC signals. 


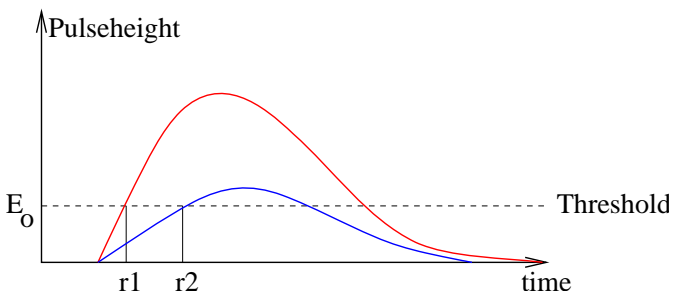

Figure 5.8: The effect of different pulseheights for a signal of constant rise time on the trigger times $(\mathrm{r} 1, \mathrm{r} 2)$ of a leading edge discriminator.

To measure the walk effect a signal with a fixed start time and well defined scintillator hit position is required, so that the distance the light travels in the scintillator is constant and does not effect the timing. A horizontal trigger detector, equipped with constant fraction discriminators which have hardware to compensate for walk effects, was positioned behind the first wall for dedicated calibration data runs to meet these requirements. The relationship between the TDC, (t), and QDC, (E), signals can then be approximated by the relation[71] :

$$
t=a+\frac{b}{\sqrt{E}}
$$

where $a$ is a constant time offset between the polarimeter TDC and the reference scintillator and $b=r \sqrt{E_{\circ}}$, with $r$ being the rise-time of the pulse and $E_{\circ}$ the discriminator threshold. The square root is due to the assumption that the pulse initially rises quadratically with time which is approximately true.

To calibrate the first-wall walk correction, the polarimeter trigger (sec. 4.7) was set to accept coincidences between any polarimeter scintillator and the trigger detector. The electron beam was incident on a polyethylene target, yielding particles with a large dynamic range of energies to pass through the polarimeter. The fixed position of the trigger detector gave a fixed hit position in the polarimeter scintillator. Plots were then made of $E$ versus $t$ for each scintillator and used to determine the parameters of equation 5.3. These were then stored in the configuration files where they were accessed during the data reconstruction. An example of a TDC spectrum before and after the walk correction is shown in figure 5.9.

For the second-wall walk correction the trigger required a coincidence between a first wall scintillator, a second wall scintillator and the trigger detector. Here to select hits from a fixed point on the scintillator, a cut on the difference of the two second wall TDCs was required. However, the situation for the second wall 

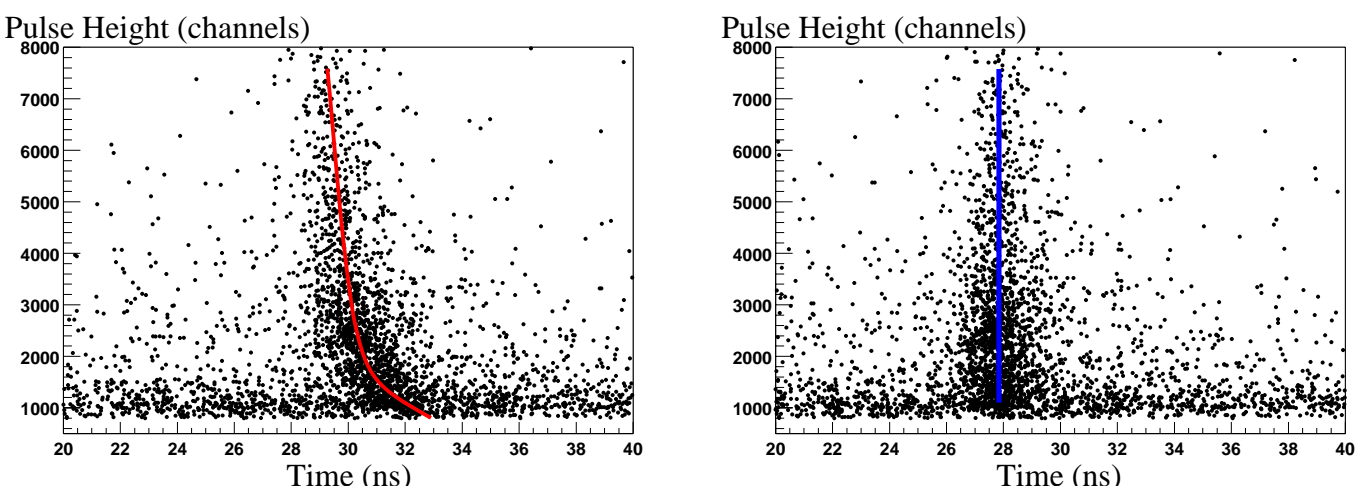

Figure 5.9: The plot on the left shows the leading edge walk effect. The plot on the right shows the same data after the walk correction.

turned out to be more complicated as the walk parameter $b$, varied with position along the bar. This is a dispersion effect of the scintillation photons traveling up to $1.8 \mathrm{~m}$ through the scintillator bar. To account for this the parameter $\mathrm{b}$ was fitted as a function of the hit position $(x)$ assuming an exponential dependence :

$$
b(x)=b_{\circ} e^{\frac{-x}{\mu}}
$$

To achieve a reasonable accuracy the parameter $\mu$ was taken as a mean for all scintillator bars, whereas the $b$ 。 parameters were bar dependent. The final calibration result for $\mu$ was around $300 \mathrm{~cm}$, implying a relatively small exponential variation along the bar.

\subsubsection{Spatial Calibrations}

The spatial calibration allowed the positions of the neutron interactions to be evaluated in the reference frame of the neutron polarimeter. This was defined so that the $\mathrm{x}$-axis was parallel to the horizontal second-wall scintillator bars (perpendicular to the first wall bars). The y-axis was parallel to the vertical first-wall bars (perpendicular to the second) and the z-axis was perpendicular to both in the radial direction from the target (fig. 4.12). The degree of segmentation of the first and second walls was sufficient so that, with relatively small errors, the $\mathrm{z}$ coordinate of a neutron interaction was given by the mean $\mathrm{z}$ position of the hit scintillator. The $\mathrm{x}$ coordinate of an interaction in the first wall and the $\mathrm{y}$ 
coordinate in the second wall was determined in a similar way.

The positions of the polarimeter walls, stands and magnet were surveyed using a goniometer from the target position and using the known dimensions of the polarimeter components, this technique gave errors of the order of a $\mathrm{cm}$. The positions of the individual bars were measured with respect to the stands to an accuracy of a few $\mathrm{mm}$. The third coordinate which corresponds to the hit position along the scintillator bar, can be determined via a calibration of the time difference between the two photomultiplier signals.

The time from an electron being scattered in the target (effectively the start time for the polarimeter TDCs) until an electronic pulse stops the TDCs of the hit scintillator can be described by the following equations:

$$
\begin{gathered}
t_{1}=t_{\circ}+\frac{x_{1}}{c_{\text {scint }}}-t_{A}+\delta t_{1} \\
t_{2}=t_{\circ}+\frac{\left(L-x_{1}\right)}{c_{\text {scint }}}-t_{A}+\delta t_{2}
\end{gathered}
$$

Where $t_{\circ}$ is the flight time of the neutron from the target to the scintillator, $t_{A}$ is the time for the electron to travel from the target to the spectrometer trigger scintillator, $\delta t_{1,2}$ account for all the fixed delays in cables and electronics, $c_{\text {scint }}$ is the speed of light in the scintillator, $\mathrm{L}$ is the length of the scintillator and $x_{1}$ is the hit position with respect to PM1 connected to TDC1 (fig. 5.10). Subtracting $t_{2}$ from $t_{1}$ gives :

$$
\begin{aligned}
\Delta t & =t_{1}-t_{2}=\frac{1}{c_{\text {scint }}}\left(2 x_{1}-L\right)+\left(\delta t_{1}-\delta t_{2}\right) \\
\Rightarrow x_{\circ} & =x_{1}+\frac{L}{2}=\frac{c_{\text {scint }}}{2}\left\{\Delta t-\left(\delta t_{1}-\delta t_{2}\right)\right\}
\end{aligned}
$$

where $x_{\circ}$ is the hit position relative to the centre of the bar, which is the required coordinate in the neutron polarimeter frame. The parameters required to calibrate $\Delta t$ for each scintillator are then $c_{\text {scint }}$ and $t_{\text {off }}=\left(\delta t_{1}-\delta t_{2}\right)$.

\section{First-Wall}

Dedicated beam time was devoted to the spatial calibrations. For the first-wall the $\mathrm{y}$-coordinate is obtained from the TDC difference and thus the trigger scintillator 


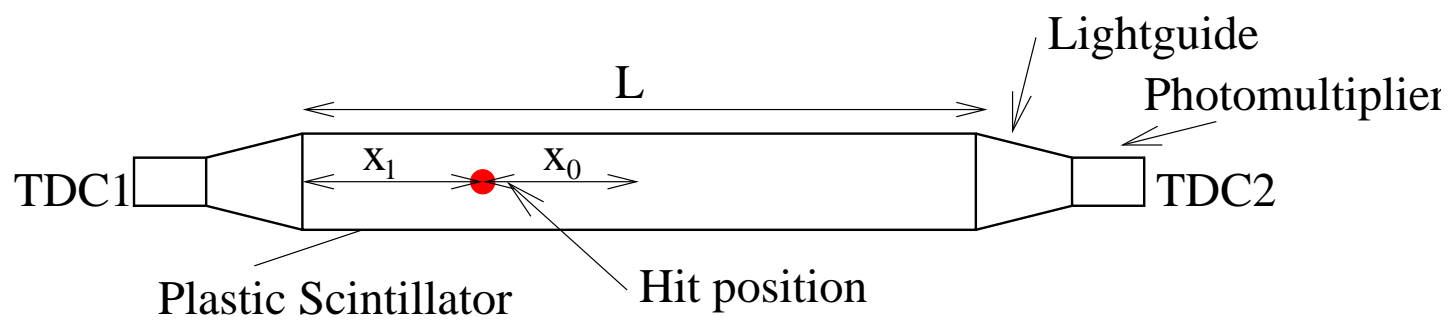

Figure 5.10: Schematic of neutron detector.

detector was aligned horizontally to select signals in the scintillator bar which were from a fixed position. The height of the trigger detector was then varied and the centroid of the distribution of $\Delta t$ plotted as a function of trigger detector height. The parameters $c_{\text {scint }}$ and $t_{\text {off }}$ were then determined from the gradient and crossing point of this highly linear functional dependence.

Additional data taken for the elastic $H\left(e, e^{\prime} p\right)$ reaction allowed a check of this procedure. Once the electron trajectory has been reconstructed by the spectrometer the momentum of the recoiling proton is known accurately and therefore its hit position in the first wall detectors can be predicted. A comparison of these hit positions with those reconstructed from the calibrated polarimeter showed discrepancies of $\sim \mathrm{cm}$.

The uncertainties on the first wall position were estimated as $\sigma_{x}=1.44 \mathrm{~cm}$, $\sigma_{y}=1.2 \mathrm{~cm}$ and $\sigma_{z}=2.17 \mathrm{~cm}$.

\section{Second-Wall}

The position calibration of the second wall was done using cosmic rays and did not require the electron beam. A trigger detector was placed at various positions perpendicular to the length of the scintillator and a coincidence was demanded between the trigger detector and the second-wall to record any cosmic rays passing through both. Again by plotting the position of the trigger detector versus $\left(t_{1}-t_{2}\right)$ the parameters $c_{\text {scint }}$ and $t_{\text {off }}$ were found.

The uncertainties on the second wall position were estimated as $\sigma_{x}=2.00 \mathrm{~cm}$, $\sigma_{y}=5.77 \mathrm{~cm}$ and $\sigma_{z}=2.89 \mathrm{~cm}$.

\subsubsection{Time of Flight Calibrations}

An actual time of flight from a neutron exiting the target until it hits the scintillator can be reconstructed by adding equations 5.4 and 5.5: 


$$
\begin{gathered}
T=t_{1}+t_{2}=2 t_{\circ}+\frac{L}{c_{\text {scint }}}+\left(\delta t_{1}+\delta t_{2}\right)-2 t_{A} \\
\Rightarrow t_{\circ}=\frac{T}{2}+T_{\text {off }}+t_{A}
\end{gathered}
$$

As $t_{A}$ is already known from the spectrometer calibrations all that is required is the constant offset $T_{\text {off }}$, which accounts for the time it takes the light to travel in the scintillator and electronic delays. For this we need a reaction with a known time of flight, $t_{\circ}$.

\section{First-Wall}

The time of flight of protons from elastic $H\left(e, e^{\prime} p\right)$ scattering can be used to give a known $t_{0}$, as the momentum of the proton may be deduced from the electron detected in the spectrometer. From this momentum, the time of flight to the polarimeter can be calculated and $T_{\text {off }}$ may then be found via equation 5.6.

\section{Second Wall}

Again the situation for the second-wall is more complicated. The elastic $H\left(e, e^{\prime} p\right)$ reaction cannot be used as the protons will interact with the first wall giving an uncertainty in momentum and hence the time of flight between the first and second walls. Instead we calibrated the second wall relative to the first-wall using minimum ionising electrons. As they effectively travel at the speed of light their flight time between the first and second walls can be accurately determined :

$$
t_{\circ 2}=t_{\circ 1}+t_{12}
$$

where $t_{\circ 1}$, the absolute time of flight to the first wall, is known after the first wall has been calibrated. $t_{12}$ the time of flight of the electrons between first and second walls is given by $t_{12}=\frac{r_{12}}{c}$ where $r_{12}$ is the distance between hit positions in the first and second wall. As $t_{\circ 2}$ is now known the offset can be determined as for the first wall. Figure 5.11, shows the distribution of the calculated time of flight for relativistic particles between the two walls, minus the actual time of flight measured after the calibrations. The sharp peak at zero, associated with real relativistic particles, shows that the relative calibration is correct. 


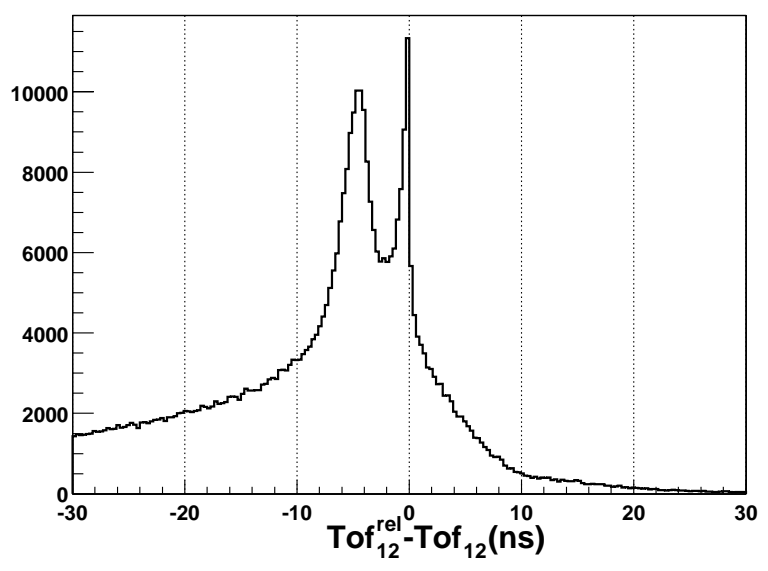

Figure 5.11: Time-of-flight for relativistic particles $\left(\frac{r_{12}}{c}\right)$ between the first and second walls minus the measured time-of-flight. The peak at zero is relativistic particles. The peak around $-5 \mathrm{~ns}$ is neutron scattering events.

\subsubsection{Energy Deposited in the First-Wall Scintillators}

An approximate $E_{d e p}^{1}$ calibration was adopted to allow a comparison between the real data and the simulation. This calibration does not effect the asymmetry analysis. The energy deposited in the scintillators was determined for each bar by taking the geometric mean of the QDC readings for each photomultiplier attached to the bar: $E_{d e p}^{1}=\sqrt{Q D C_{1} \cdot Q D C_{2}}$. This was then histogrammed for events with protons passing through the first wall and then compared to a similar histogram taken from the simulation using the standard GEANT4 proton energy loss model (fig. 5.12). As explained in section 5.1.4 the simulation gives $E_{d e p}^{1}$ in units of $\mathrm{MeV}_{e e}$ so by matching the peak position of the simulated and real proton $E_{d e p}^{1}$ peak the real data can be calibrated with a linear function in units of $\mathrm{MeV}_{e e}$. To compare to the simulation the real data was divided by a factor of $\frac{5100}{53} \simeq 96$.

\subsection{Calculating Precession Angle}

An important part of the analysis is the calculation of the neutron spin precession as it passes through the vertical field of the dipole magnet. The polarisation ratio is derived from the zero-crossing angle $\chi_{\circ}$ (eqn. 4.6) and is therefore sensitive to errors in the precession angle, $\chi$.

A neutron with a path $L(x, y, z)$ through a magnetic field of strength $\mathrm{B}\left(0, B_{y}, 0\right)$ will have its spin precessed in the $x$ - $z$ plane by an angle [74]: 

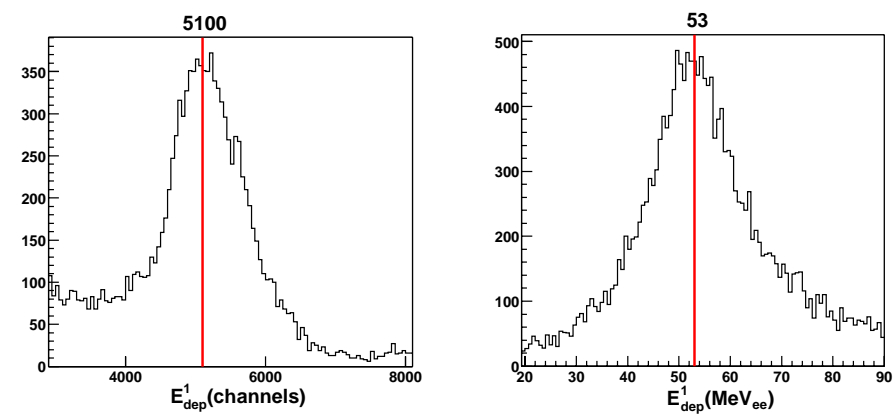

Figure 5.12: $E_{\text {dep }}^{1}$ from protons passing through the first wall of the polarimeter for real data (left) and simulated data (right).

$$
\chi=\frac{2 \mu_{n}}{\hbar c} \frac{1}{\beta_{n}} \int_{L} B d l
$$

Where $\mu_{n}$ is the neutron magnetic moment and $\beta_{n}$ is the neutron velocity reconstructed from the electron four-vector and the hit position in the polarimeter (sec. 5.4.3). $\beta_{n}$ could also be calculated from the time of flight of the neutrons to the first wall of the polarimeter, but this would have a larger uncertainty.

\subsubsection{Dipole-Field Integral}

To determine the field integral $\int_{L} B d l$ for an event [75], the path of the neutron through the field must be known. The path of the neutron through the field was a straight line from the target (assumed point-like) to its hit position $\vec{P}_{1}$, in the first wall of the polarimeter, which had polar coordinates $\theta_{1}$ and $\phi_{1}$ (recreated in sec. 5.4.1). $\vec{P}_{1}\left(\theta_{1}, \phi_{1}\right)$ uniquely determines the neutron's point of entry, in the x-y plane, to the magnet, $\vec{P}_{M}\left(\theta_{1}, \phi_{1}\right)$.

We have then that $\int_{L} B d l=B_{\text {int }}\left(x_{M}, y_{M}\right)=B_{\text {int }}\left(\theta_{1}, \phi_{1}\right)$. Where $\left(\theta_{1}, \phi_{1}\right) \rightarrow$ $\left(x_{M}, y_{M}\right)$ through a standard polar-Cartesian coordinate transformation. The integrated field for the seven different magnetic currents used in the experiment was mapped for the full range of $x_{M}$ and $y_{M}$ using the MAFIA 3D magnetic field simulation package. In this, the dipole field was calculated at 200000 mesh points in a volume defined by one quarter of the magnetic gap (fig. 5.13). The symmetry of the dipole geometry then allowed these results to be extrapolated for the full volume. The mesh points were separated by distances of about $1 \mathrm{~cm}, 0.98 \mathrm{~cm}$ and $4.88 \mathrm{~cm}$ in the $\mathrm{X}, \mathrm{Y}$ and $\mathrm{Z}$ directions respectively and continuous variation of the field was achieved by a smooth interpolation between the mesh points. The 


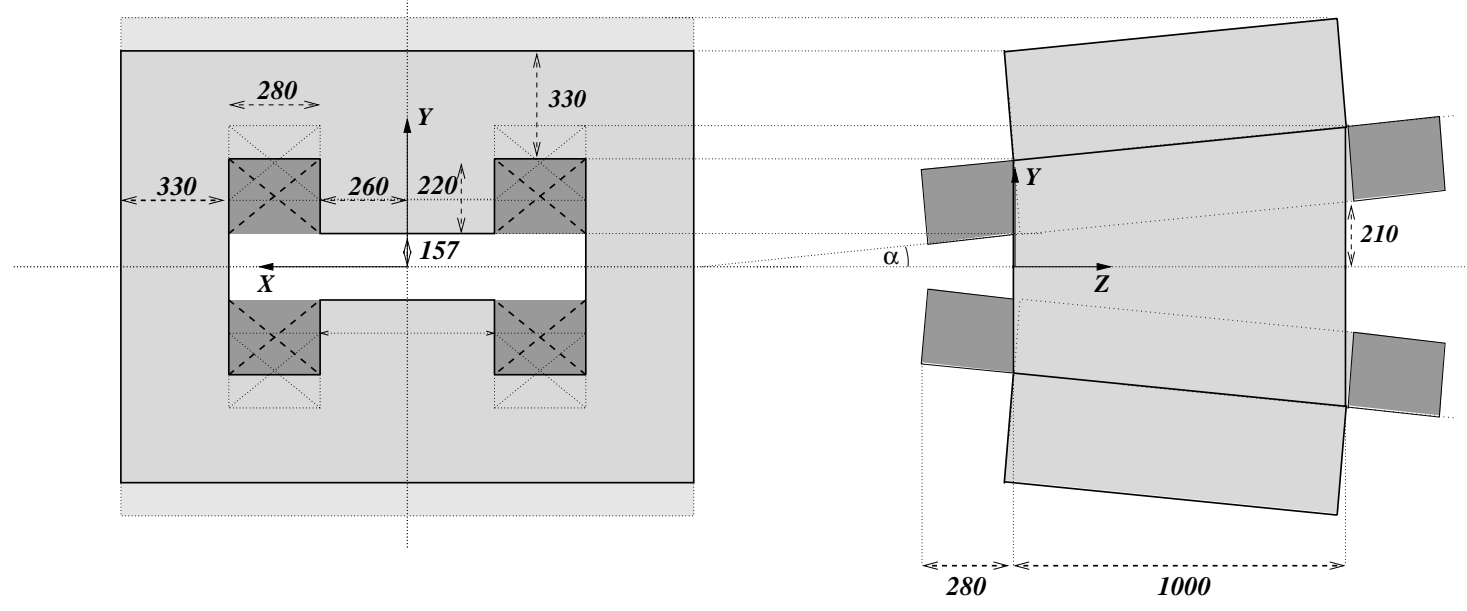

Figure 5.13: Scale diagram of the dipole magnet, also shown is the Cartesian coordinate system used in the text. Left is the view from the target (front). Right is the view from the side.

3-dimensional field map produced was then used to integrate the flux along a given path defined by $x_{M}$ and $y_{M}$, giving the field integral $B_{i n t}\left(x_{M}, y_{M}\right) . B_{i n t}$ was calculated for 2079 entry points for each magnetic field setting and the results were written to input files for the analysis code which determined $\left(x_{M}, y_{M}\right)$ on the neutron flight path and then found the closest field integral for that event.

In addition to simulating the field in this way it is important to measure at least part of it to check the accuracy of the 3D simulation. This was performed using a Hall probe to map the field in the x-z plane, measuring at points approximately $2 \mathrm{~cm}$ apart in each direction. The error in measured field strength was estimated to be of the order of $1 \%$. This map was then analysed in a similar manner to the simulated field map (smoothed and integrated) and the two path integrals compared for flight paths in the $\mathrm{x}-\mathrm{z}$ plane. This procedure was repeated for a number of different paths, $\mathrm{x}-\mathrm{z}$ planes and currents. The discrepancies ranged from effectively zero at the centre of the magnet gap to $2 \%$ at the edges of the pole faces for high currents. Also measurements were made of the small $\mathrm{x}$ and $\mathrm{z}$ field components, which were shown to contribute less than $1 \%$ to the spin precession and therefore were neglected. Overall the error in the field integral was determined to be $2 \%$ [52]. 


\subsection{Event Reconstruction}

COLA ++ is the in-house analysis software for experiments performed in the MAMI A1 spectrometer hall. For the $G_{E}^{n}$ analysis we used the standard Spectrometer A reconstruction code to recreate the scattered electron four-momentum, but new code had to be developed to deal with the neutron polarimeter [72]. Once $\mathrm{COLA}++$ had performed the initial event reconstruction and the relevant kinematic variables were stored in a ntuple ${ }^{2}$ further reconstruction was done using a ROOT [63] based analysis code. At this stage multiple polarimeter hits were sorted and four-vectors calculated. The resulting TGEnEvents were stored in a TTree $^{3}$ for further analysis.

\subsubsection{Neutron Polarimeter Reconstruction}

COLA ++ accessed the raw data files, which for the polarimeter consist of QDC and TDC values. Using an object orientated methodology these were passed to the appropriate scintillator bar object, each of which had two ADC and two TDC variables corresponding to information read out from the actual bar. A bar object was created for every scintillator in the polarimeter and these objects fed the reconstruction of a neutron event performed by the "npol.cc" code. This procedure is outlined below:

- The data was checked to see if there were valid TDC and QDC values for the bar, i.e not "overflow" or "underflow" entries.

- The QDC pedestals were then subtracted and the new values were checked to see if they corresponded to a real signal. i.e. if they were still greater than zero.

- The two QDC values were combined to give the pulse height which is proportional to the energy deposited in the bar, where, $E_{\text {dep }}=\sqrt{Q D C 1 \cdot Q D C 2}$.

- The walk correction was applied for the TDCs (sec. 5.2.3).

- The TDCs were combined to give the absolute time of flight of the neutron and hit position along the bar:

\footnotetext{
${ }^{2} \mathrm{An}$ ntuple is a store of multi-parameter events with information for each event stored as an array of real numbers.

${ }^{3} \mathrm{~A}$ TTree is a store of multi-parameter events with information for each event stored as an array containing different ROOT class objects (e.g. vector, matrix).
} 


$$
\begin{gathered}
\text { tof }=\frac{T D C 1+T D C 2}{2}+T_{o f f}+t_{A} \\
\text { pos }=t d c s c a l e \cdot c_{\text {scint }} \cdot\left(T D C 1-T D C 2+t_{o f f}\right)
\end{gathered}
$$

Where the offsets ( $T_{\text {off }}$ and $\left.t_{o f f}\right), t_{A}$, tdcscale and the speed of light in the scintillator $c_{\text {scint }}$, had been determined through the calibrations described in section 5.2.4.

- The physical position of the bar in the frame of the neutron polarimeter was read from an input file to give the other two Cartesian coordinates of the hit, giving a hit position vector.

- The polar coordinates of the hit position were calculated.

- Cuts were made on the time of flight to select events inside the electronic timing gates of the event trigger, allowing both real and random events to be analysed and rejecting "junk" events caused by transient effects at gate edges etc.

The bars were then grouped depending on which part of the polarimeter they represented, veto or neutron detector, first or second wall, top or bottom frame.

- Hit neutron detector bars were checked to see if they were in coincidence with the veto detectors in front of them, i.e. was the hit caused by a charged particle?

- If they were in coincidence, a flag was set to identify them as proton events. If they were not in coincidence, the flag was set to identify them as neutrons.

- The hit position in the first wall was used to determine the magnet field integral associated with the neutron flight path. (sec. 5.3).

- The event information was then written to an ntuple if there was a "good" hit in a bar for both the first and second wall.

Information was given to the ntuple for each bar : the time of flight, the pulseheight, the polar coordinates of the hit position, the field integral, the bar number and the proton/neutron flag. 

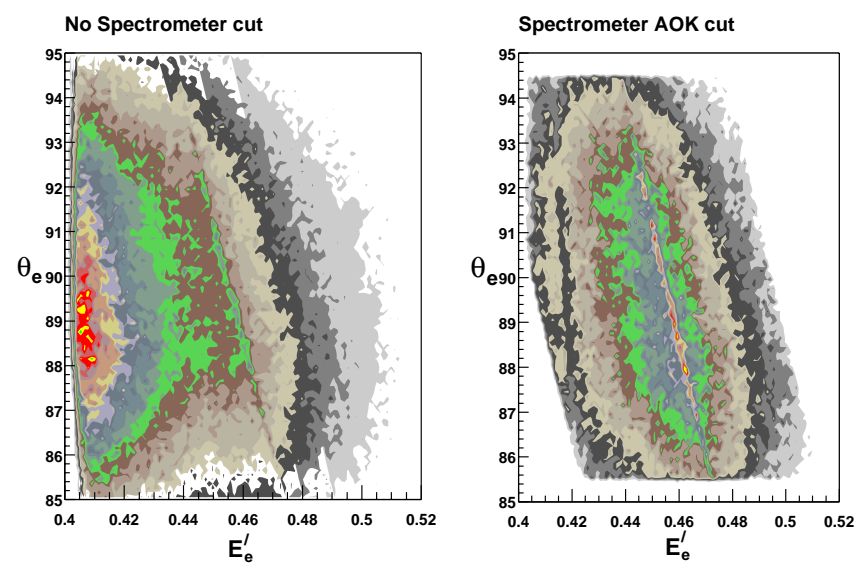

Figure 5.14: The effect of the AOK cut on scattered electron angle $\left(\theta_{e}\right)$ versus energy $E_{e}^{\prime}$.

In addition to the polarimeter information the four-momentum of the virtual photon, a flag indicating a valid electron event and the beam helicity were given to the ntuple for each event. The ntuples were then analysed separately using the ROOT data-analysis package.

\subsubsection{Electron Spectrometer}

Section 4.4, described the operation and calibration of the magnetic spectrometer. The scattered electron four vector was reconstructed as part of the COLA ++ analysis. The resulting angle $\theta_{e}$ versus energy $E_{e}^{\prime}$ distribution is shown in figure 5.14 , before(left) and after(right) the application of the "AOK" cut and without any cuts on the polarimeter. AOK is a combination of cuts : a cut on the spectrometer-polarimeter coincidence time to reduce the random background; a cut on the vertex reconstruction to reject events from the target walls and a cut on a signal from the threshold Cerenkov detector to veto $\pi^{-}$. The sharp line in both spectra correspond to elastic electron-proton scattering events which were due to a small amount of Hydrogen contamination in the target cell. In the right hand plot the remaining quasi-elastic events are distributed around this line as a result of the Fermi motion of the struck nucleon in deuterium.

\subsection{3 $G_{E}^{n}$ Events}

The purpose of this stage in the analysis was to create ROOT TTrees that could be analysed to create $\phi_{n}^{\prime}$ distributions and thus asymmetries, with a variety of 
kinematic cuts and a random background subtraction. The ntuple output from the COLA ++ analysis could contain multiple hits in the same polarimeter wall for each event. In the case of multiple hits in neighbouring bars it was assumed that each came from the same scattering interaction, in which case the energy deposited was summed and they were stored as just one hit. If more than one bar was hit and they were not neighbouring, we could not easily decide a priori which if any was a true hit as opposed to a random and we analysed each separately. The real neutron would contribute to a scattering asymmetry, while randoms would produce a background, that had to be subtracted to leave the real signal at a later stage. Thus if there were two of these hits in the first wall and one in the second, two events were considered. If there were two in the first and two in the second, four events were created as each first wall hit has to be combined with each second wall hit, and so on.

Also two sets of TTrees are required to separate the case of detecting a proton in the second wall from detecting a neutron. From now on these two cases will be referred to as NP and NN events respectively.

A single $G_{E}^{n}$ event in the TTree consists of four four-vectors and additional polarimeter information. The event variables are explained in table 5.1.

\section{Particle Four-Momenta}

The energy and momentum of the virtual photon $q$, was calculated from the beam and scattered electron four-vectors : $q=e-e^{\prime}$.

Two four-vectors $n_{i n}$ and $n_{i n}^{\gamma}$ were determined for the neutron travelling from the target to the first wall. The first had its energy calculated from the time of flight measurement, the second from the hit position and virtual photon kinematics. The momentum direction for both was given by the vector from the target to the hit position in the first wall, the polar coordinates $\left(r_{1}, \theta_{1}, \phi_{1}\right)^{4}$ having already been calculated (sec. 5.4.1). The energy $E_{n}$ of $n_{\text {in }}$ was calculated for $t=t_{1}$ and $r=r_{1}$ using :

$$
\begin{gathered}
\beta=\frac{r}{t . c} \\
E=\frac{m_{n}}{\sqrt{1-\beta^{2}}}
\end{gathered}
$$

\footnotetext{
${ }^{4}$ The polar coordinates $r_{1}, \theta_{1}, \phi_{1}$ are given in the neutron polarimeter frame (fig. 4.9).
} 


\begin{tabular}{|c|c|}
\hline Variable & Meaning \\
\hline$q$ & Four-vector for the virtual photon. \\
\hline$n_{\text {in }}$ & $\begin{array}{l}\text { Four-vector for the neutron between the target and the } \\
\text { 1st wall. With energy calculated from tof } 1 \text {. }\end{array}$ \\
\hline$n_{\text {scat }}$ & $\begin{array}{l}\text { Four-vector for the neutron after being scattered in the } \\
\text { first wall. With energy calculated from tof } 1 \text { and tof } 2 \text {. }\end{array}$ \\
\hline$n_{i n}^{\gamma}$ & $\begin{array}{l}\text { Four-vector for the neutron between the target and the } \\
\text { 1st wall. With energy calculated from Spectrometer A. }\end{array}$ \\
\hline$\theta_{n}^{\prime}$ & $\begin{array}{l}\text { Neutron scattering angle between first second walls. See } \\
\text { figure } 4.9\end{array}$ \\
\hline$\phi_{n}^{\prime}$ & Neutron azimuthal scattering angle. \\
\hline$t_{1}$ & Time of flight from target to 1st wall. \\
\hline$t_{2}$ & Time of flight from target to 2nd wall. \\
\hline$E_{\text {dep }}^{1}$ & Energy deposited in the 1st wall. \\
\hline$E_{d e p}^{2}$ & Energy deposited in the 2nd wall. \\
\hline$i d_{1}$ & $\begin{array}{l}\text { Number of the 1st wall scintillator bar the scattering } \\
\text { occurred in. }\end{array}$ \\
\hline$i d_{2}$ & $\begin{array}{l}\text { Number of the 2nd wall scintillator bar the scattering } \\
\text { occurred in. }\end{array}$ \\
\hline$h p_{1}$ & $\begin{array}{l}\text { Hit pattern for the 1st wall. Shows how many bars were } \\
\text { hit and the position of other hit bars relative to this one. }\end{array}$ \\
\hline$h p_{2}$ & Hit pattern for the 2nd wall. \\
\hline$n p_{1}$ & $\begin{array}{l}\text { Shows whether } 1 \text { st wall hit is in coincidence with veto } \\
\text { or not. i.e. neutron }(=1) \text { or proton hit }(>1) \text {. }\end{array}$ \\
\hline$n p_{2}$ & $\begin{array}{l}\text { Shows whether } 2 \text { nd wall hit is in coincidence with veto } \\
\text { or not. i.e. neutron }(=1) \text { or proton hit }(>1) \text {. }\end{array}$ \\
\hline veto $_{1}$ & Records how many first wall veto detectors fired. \\
\hline$\overline{B_{\text {int }}}$ & $\begin{array}{l}\text { The integrated field the neutron experiences through the } \\
\text { magnet. }\end{array}$ \\
\hline$N o S_{1}$ & $\begin{array}{l}\text { Indicates wether the } 1 \text { st wall hit was from a prompt tof1 } \\
\text { region }(=0) \text { or a random region }(\neq 0) \text {. }\end{array}$ \\
\hline $\mathrm{NoS}_{2}$ & $\begin{array}{l}\text { Indicates wether the } 2 \text { nd wall hit was from a prompt } \\
\text { tof2 region }(=0) \text { or a random region }(\neq 0) \text {. }\end{array}$ \\
\hline Case & $\begin{array}{l}\text { Seperates events into }+/- \text { helicity and } 0<\phi_{n}^{\prime}<\pi ; \pi< \\
\phi_{n}^{\prime}<2 \pi \text { combinations. }\end{array}$ \\
\hline
\end{tabular}

Table 5.1: $G_{E}^{n}$ event variables. 


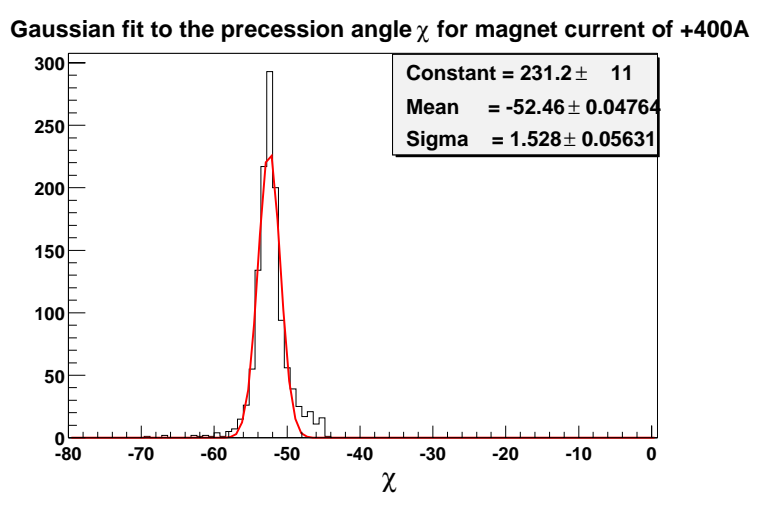

Figure 5.15: A Gaussian fit to $\chi$ (deg.) for data taken with the precession magnet current set to $400 \mathrm{~A}$.

The energy of $n_{i n}^{\gamma}$ was calculated from $\gamma, \theta_{1}$ and $\phi_{1}$ (see appendix B.2).

After being scattered in the first wall the neutron $n_{s c}$, had a momentum along $\underline{v}_{1}-\underline{v}_{2}$ where $\underline{v}_{1(2)}$ is the hit position on the first (second) wall. This resulting vector was then rotated into the reference frame $x_{i}^{n}$ (fig. 4.9 and eqn. 5.9), so that the scattering angles of interest $\theta_{n}^{\prime}$ and $\phi_{n}^{\prime}$ are given in this frame. The energy was calculated using equation 5.8, with $t=t_{2}-t_{1}$ and $r=\left|\underline{v}_{1}-\underline{v}_{2}\right|$.

$$
\underline{z_{n}}=\underline{\widehat{p_{n}}} \quad \underline{y_{n}}=\underline{\widehat{p_{n}}} \times \underline{\widehat{q}} \quad \underline{x_{n}}=\underline{\widehat{y_{n}}} \times \underline{\widehat{z_{n}}}
$$

\section{Mean Precession Angles}

The precession angle for each event was calculated from eqn. 5.7. The mean precession angle for each magnetic field was taken from a Gaussian fit to the precession angle distribution from the accepted events. An example of the fit for a magnetic current of $+400 \mathrm{~A}$ is shown in figure. The systematic uncertainty on $\chi$ is the same as that of $B_{\text {int }}$ which is $2 \%$.

\section{Hit Pattern}

As explained earlier (sec. 5.4.3) it was necessary to know the number and position of hit scintillators relative to each other. This information was recorded by the variables $h p_{1}$ and $h p_{2}$ for the first and second walls respectively. Table 5.2 shows the possible values of $h p_{1,2}$. Only events with $0<h p_{1,2}<100$ were analysed 


\begin{tabular}{|c|c|}
\hline & meaning \\
\hline \hline 1 & no neighbours hit \\
\hline$+\mathrm{n}$ & $\mathrm{n}$ of the 2 bars at each side hit \\
\hline-10 & the bar has been combined with a side neigbour \\
\hline$+10 \mathrm{n}$ & $\mathrm{n}$ of the three bars behind hit \\
\hline 100 & one of the 2 bars diagonally in front hit \\
\hline 200 & bar directly in front hit \\
\hline
\end{tabular}

Table 5.2: Hit Pattern decoding information
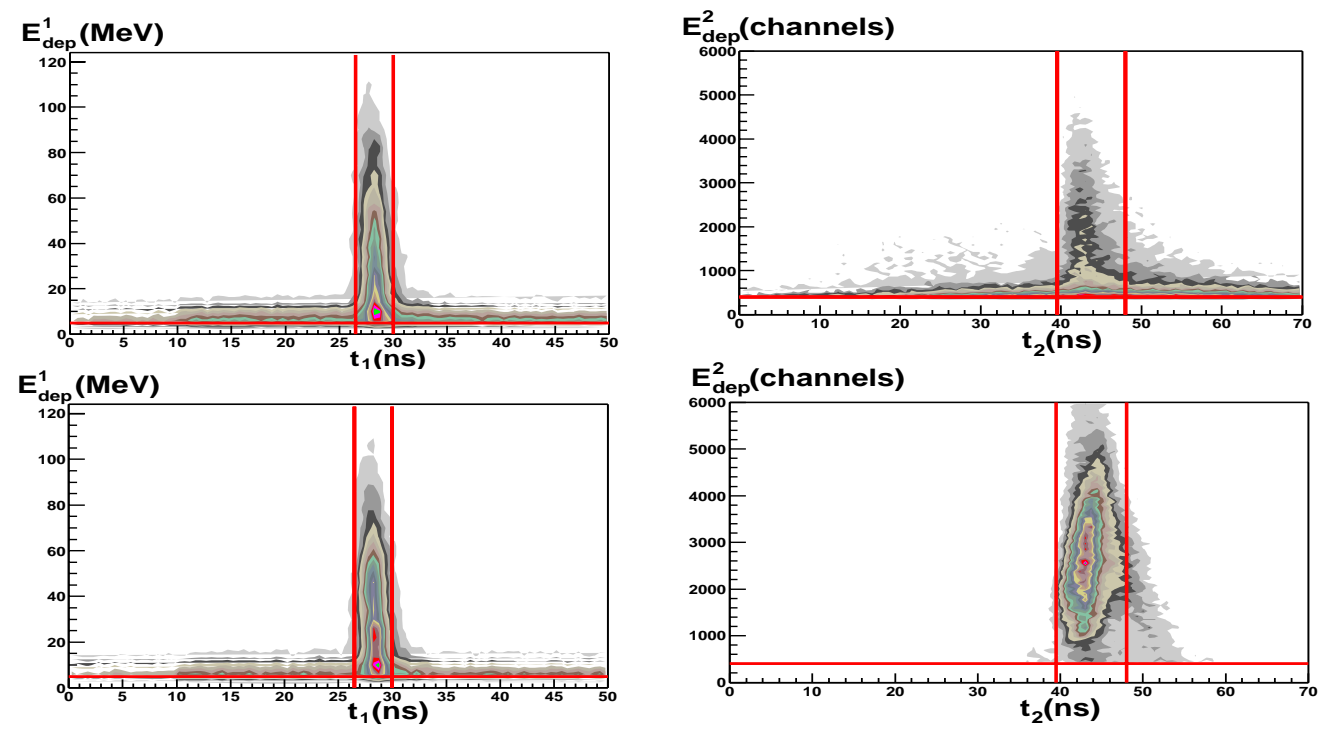

Figure 5.16: The prompt time cuts and $E_{\text {dep }}$ threshold are shown by the red lines. The top two panels show $E_{\text {dep }} v s . t$ for the first and second wall NN events, the bottom two panels show the NP events.

as other numbers correspond to the same interaction and analysing such events would result in double counting. In these cases the energy deposited in the neighbouring scintillator is added to the analysed event and the hit position is averaged between the two.

\section{Prompt and Random Events}

In order to apply a background subtraction (sec. 5.5.1) it was necessary to define prompt timing regions where we could expect to find quasi-elastically scattered neutrons from the target and random timing regions, where random coincidences only were expected. To allow a random background region to be analysed under the application of kinematic cuts, the time of flight for the random region had to 
be offset to fall in the same range as the prompt region and thus have the same range of kinetic energy. The variable $\mathrm{NoS}_{1,2}$ (table 5.1) refers to the degree of shift of the random coincidence region in order to bring it into the same range as the prompt region and is by definition equal to zero for the prompt region.

There were four possible combinations of prompt and random events from the two polarimeter walls, i.e. first-wall prompt, second-wall prompt (PP); first-wall prompt, second-wall random (PR); first-wall random, second-wall prompt (RP); first-wall random, second-wall random (RR). An output TTree containing all the variables shown in table 5.1 was required for each combination.

Figure 5.16 illustrates the prompt cuts used in the analysis: $26.5<t_{1}<30$ ns and $39.5<t_{2}<48 \mathrm{~ns}$. The plots show the energy deposited in the scintillator versus the time of flight for both the first and second wall and NN and NP type events. The band of low $E_{d e p}$ which span all of the time-of-flight spectra are random coincidences mainly from $\gamma$-ray interactions, the effect of these events on the measured asymmetry is discussed in the following section. A low threshold cut of $E_{d e p}^{1}>5 \mathrm{MeV}$ and $E_{\text {dep }}^{2}>400$ channels was also applied, this gets rid of a large amount of the background while losing very few real events.

\subsection{Calculating Asymmetries}

The previous section described how TTrees were produced with the relevant event information for creating and analysing the asymmetry $A(\chi)$ described by equation 4.7. At this point a total of 56 TTrees were produced : $2 \times 4 \times 7$ $=($ neutron in second-wall + proton in second-wall $) \times($ number of prompt $/ \mathrm{random}$ regions $) \times($ number of magnetic fields $)$. For each magnetic field an asymmetry was produced along with a precession angle. The process of calculating these asymmetries is described in the rest of this section.

Figure 5.17 shows an idealised $\phi_{n}^{\prime}$ distribution, where $\phi_{n}^{\prime}$ is the azimuthal scattering angle of the incident neutron (fig. 4.9). The variable $X=\alpha_{\text {eff }} P_{e} P_{x}^{n}$ is the product of the analysing power, electron beam polarisation and the transverse neutron polarisation, (eqn. 4.7) . In the case of the experimental data we use yields, instead of cross-sections, as the solid angles, luminosity etc. cancel in the asymmetry. The relation $N_{\text {full }}\left(\phi_{n}^{\prime}\right)=N_{\circ}\left(1+X \sin \phi_{n}^{\prime}\right)$ (red line), shows the ideal distribution for a polarimeter with full $\phi_{n}^{\prime}$ acceptance. Our polarimeter had a limited acceptance related to the geometry of the two frames of scintillator in the second wall. In this case (blue line), we get $N_{\text {exp }}\left(\phi_{n}^{\prime}\right)=N_{\text {full }}\left(\phi_{n}^{\prime}\right) \Omega\left(\phi_{n}^{\prime}\right)$ where 


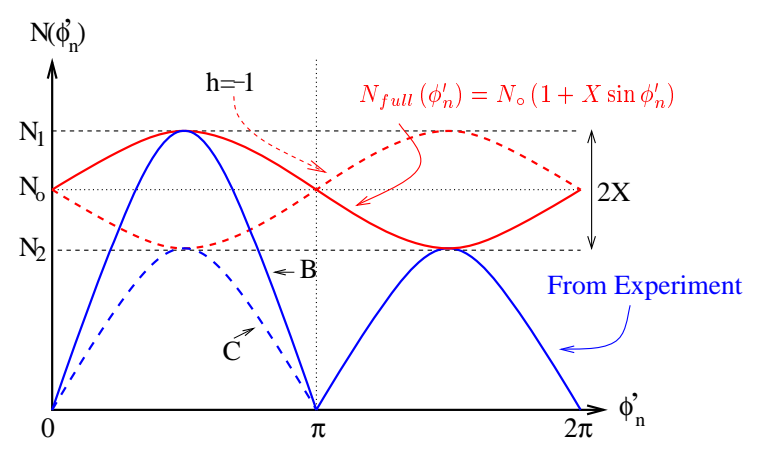

Figure 5.17: Idealised diagram of the $\phi_{n}^{\prime}$ distribution. The red line shows the case for full $\phi_{n}^{\prime}$ acceptance, while the blue line for an acceptance similar to the polarimeter. The full lines are for beam helicity $\mathrm{h}=+1$ and the dashed for $\mathrm{h}=-1$. The experimental distributions go to zero at $0, \pi$ and $2 \pi$ because there is no second wall acceptance at these angles $(\Omega=0)$.

$\Omega\left(\phi_{n}^{\prime}\right)$ is the fraction of the $\phi_{n}^{\prime}$ range within the acceptance. Helicity flipping $(h= \pm 1)$, of the beam causes $P_{x}^{n} \rightarrow-P_{x}^{n}$ and so $N_{\text {full }}$ flips to the dashed curve and $N^{+}\left(\phi_{n}^{\prime}\right) \rightarrow N^{-}\left(\phi_{n}^{\prime}+\pi\right)$. When the yields are combined to give B and $\mathrm{C}$ (eqn. 5.10), with $\phi_{n}^{\prime}$ shifted by $\pi$ so $0<\phi_{n}^{\prime}<\pi$, the helicity flipping cancels any differences in geometry and counting efficiency between the two frames and so we get $\Omega_{B}\left(\phi_{n}^{\prime}\right)=\Omega_{C}\left(\phi_{n}^{\prime}\right)$ and the acceptance will cancel out in the asymmetry :

$$
A\left(\phi_{n}^{\prime}\right)=\frac{\sqrt{N^{+}\left(\phi_{n}^{\prime}\right) N^{-}\left(\phi_{n}^{\prime}+\pi\right)}-\sqrt{N^{+}\left(\phi_{n}^{\prime}+\pi\right) N^{-}\left(\phi_{n}^{\prime}\right)}}{\sqrt{N^{+}\left(\phi_{n}^{\prime}\right) N^{-}\left(\phi_{n}^{\prime}+\pi\right)}+\sqrt{N^{+}\left(\phi_{n}^{\prime}+\pi\right) N^{-}\left(\phi_{n}^{\prime}\right)}}=\frac{B\left(\phi_{n}^{\prime}\right)-C\left(\phi_{n}^{\prime}\right)}{B\left(\phi_{n}^{\prime}\right)+C\left(\phi_{n}^{\prime}\right)}
$$

The four azimuthal angular distributions used to calculate $A(\chi)$ for each magnetic field are :

$$
\begin{aligned}
& N^{+}\left(\phi_{n}^{\prime}\right) \Longrightarrow \text { positive helicity and } 0<\phi_{n}^{\prime}<\pi \\
& N^{-}\left(\phi_{n}^{\prime}\right) \Longrightarrow \text { negative helicity and } 0<\phi_{n}^{\prime}<\pi \\
& N^{+}\left(\phi_{n}^{\prime}+\pi\right) \Longrightarrow \text { positive helicity and } \pi<\phi_{n}^{\prime}<2 \pi \\
& N^{-}\left(\phi_{n}^{\prime}+\pi\right) \Longrightarrow \text { negative helicity and } \pi<\phi_{n}^{\prime}<2 \pi
\end{aligned}
$$

These were combined as in equation 5.10 to produce the distributions $B\left(\phi_{n}^{\prime}\right)$ and $C\left(\phi_{n}^{\prime}\right)$ which were subject to kinematic cuts and a random background subtraction which is described below. The statistical uncertainty of these histograms is discussed in appendix A. 

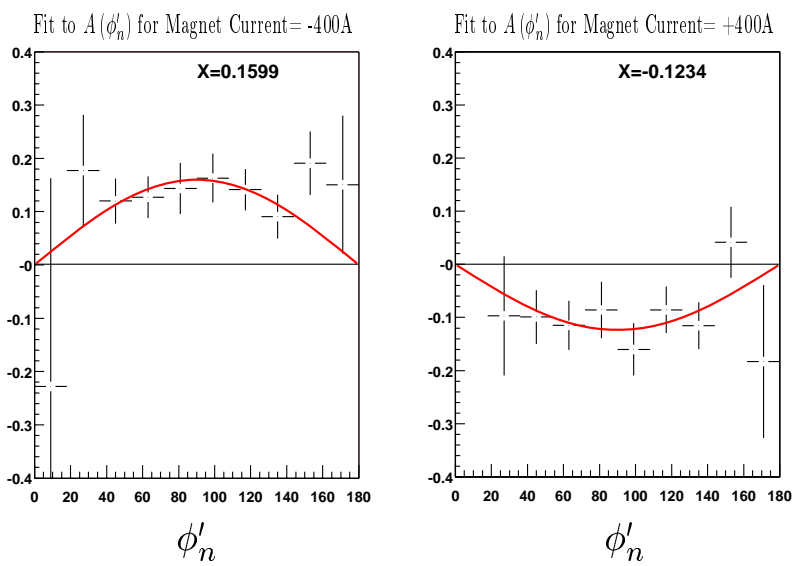

Figure 5.18: A one-parameter sine fit to the $\phi_{n}^{\prime}$ asymmetry distribution. The resulting amplitude $\mathrm{X}$ is shown.

\section{Asymmetry from fitting $A\left(\phi_{n}^{\prime}\right)$ distributions}

Rewriting equation 5.10 one obtains :

$$
\begin{aligned}
A\left(\phi_{n}^{\prime}\right) & =\frac{B\left(\phi_{n}^{\prime}\right)-C\left(\phi_{n}^{\prime}\right)}{B\left(\phi_{n}^{\prime}\right)+C\left(\phi_{n}^{\prime}\right)} \\
& =\frac{N_{\circ}\left[\left(1+X \sin \phi_{n}^{\prime}\right)-\left(1-X \sin \phi_{n}^{\prime}\right)\right] \Omega\left(\phi_{n}^{\prime}\right)}{N_{\circ}\left[\left(1+X \sin \phi_{n}^{\prime}\right)+\left(1-X \sin \phi_{n}^{\prime}\right)\right] \Omega\left(\phi_{n}^{\prime}\right)}=X \sin \phi_{n}^{\prime}
\end{aligned}
$$

A one-parameter sinusoidal fit to the histogram $A\left(\phi_{n}^{\prime}\right)$ gives the amplitude $\mathrm{X}$, which for a particular spin precession angle gives $A(\chi)$. This asymmetry is actually the product of the effective analysing power, the beam polarisation and the transverse neutron polarisation:

$$
A(\chi)=X(\chi)=\alpha_{e f f} P_{e} P_{x}(\chi)
$$

Examples of this fit for data taken with precession magnet currents of $\pm 400 \mathrm{~A}$ are shown in figure 5.18. The large increases in uncertainties at $\phi_{n}^{\prime}$ close to 0 and $180^{\circ}$ results from the finite polarimeter acceptance in azimuthal angle.

\subsubsection{Random Background Subtraction}

When detecting neutrons by time-of-flight any real neutron signal in the polarimeter will be contaminated by random coincidences with the electron spectrometer. If $B$ and $C$ are the total $\phi_{n}^{\prime}$ distributions (i.e. containing both real and ran- 


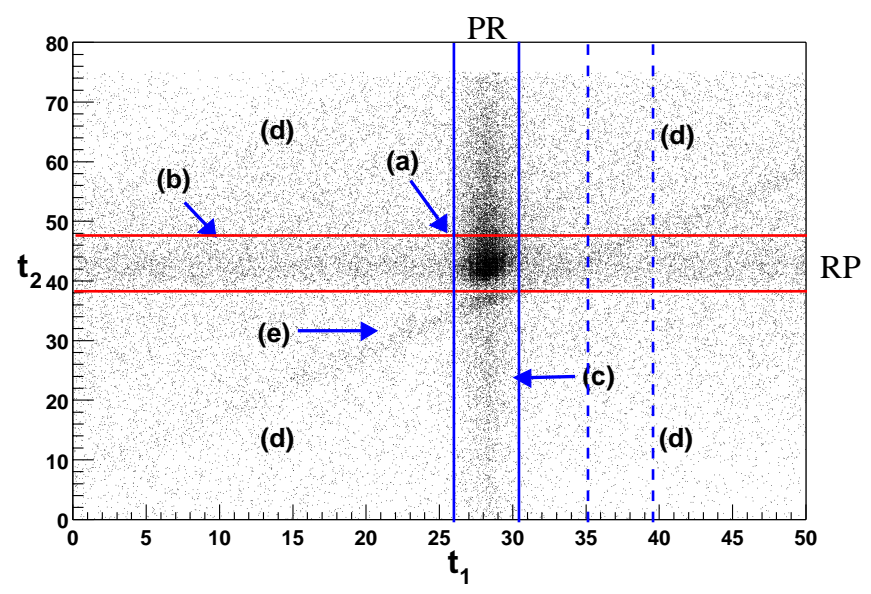

Figure 5.19: Time-of-flight to the 2 nd polarimeter wall $\left(t_{2}\right)$ versus the 1 st $\left(t_{1}\right)$ in ns. The good neutron scattering events are in the cluster marked (a), the other features are explained in the text. The full red and blue lines show the prompt timing cuts for $t_{2}$ and $t_{1}$. The resulting projections on the $t_{1}$ and $t_{2}$ axis are used to fit the RP and PR background respectively. The dashed blue line shows the region used to fit the $\mathrm{RR}, t_{2}$ region.

dom coincidences) and $R_{B, C}$ are the contribution from just random coincidences, then the real neutron scattering asymmetry and the asymmetry of all events are related via :

$$
\begin{aligned}
A_{\text {real }}= & \frac{\left(B-R_{B}\right)-\left(C-R_{C}\right)}{\left(B-R_{B}\right)+\left(C-R_{C}\right)} \\
= & \frac{A_{\text {all }}}{1-\frac{1}{r}}-\frac{A_{\text {ran }}}{r-1} \\
& A_{\text {all }}=\frac{B-C}{B+C} ; \quad A_{\text {ran }}=\frac{R_{B}-R_{C}}{R_{B}+R_{C}}
\end{aligned}
$$

and the real-to-random ratio, $r=\frac{B+C}{R_{B}+R_{C}}$. Equation 5.14 shows that random background events cause dilution of the real asymmetry by a factor of $\left(1-\frac{1}{r}\right)$. In addition any asymmetry in the background itself, $A_{\text {ran }}$, will lead to an offset from the real asymmetry. By subtracting the random contributions from the total azimuthal distributions, as in equation 5.13, both these effects are corrected.

Figure 5.19 shows the time of flight for the first and second scintillator walls plotted against each other. The horizontal band (b), shows events that have a random signal in the first wall and a real signal in the second wall (RP). The vertical band (c), is prompt events in the first wall and random in the second $(\mathrm{PR})$. The intersection of these bands is the region of real neutron scattering 
events (PP). The four regions outwith the aforementioned bands (d) contain random events in both walls $(\mathrm{RR})$. An additional diagonal band (e) is the result of a relativistic particle interacting in both the first and second wall.

For each of the different random regions (RP, PR, RR), the amount of random events polluting the real signal was estimated by a fit to time-of-flight spectra using a function parameterising the signal and background. Examples of the resulting fits are shown in figure 5.20 and details are given below :

- For RP events (b), the first wall time-of-flight spectrum $t_{1}$, was created with a cut on prompt timing events in the second wall. The random background was then approximated by a 2nd degree polynomial and the neutron signal by a Gaussian $($ panel $(\mathrm{i})): R P\left(t_{1}\right)=p_{1}+p_{2} t_{1}+p_{3} t_{1}^{2}+p_{4} \exp \left(\frac{-\left(t_{1}-p_{5}\right)^{2}}{2 p_{6}}\right)$

- For PR events (c), the second wall time-of-flight spectrum $t_{2}$, was created with a cut on prompt timing events in the first wall. $t_{2}$ was offset by $-40 \mathrm{~ns}$ so that over the prompt timing region the fit is dominated by a constant offset and minimises the contribution of the other background polynomial parameters. The random background was approximated by a 3rd degree polynomial, the small relativistic particle peak by a Gaussian and the neutron scattering signal by a three parameter Landau ${ }^{5}$ function $L\left(t_{2}\right)$ (panel (ii)) : $P R\left(t_{2}\right)=p_{1}+p_{2} t_{2}+p_{3} t_{2}^{2}+p_{4} t^{3}+L\left(t_{2}, p_{5}, p_{6}, p_{7}\right)+p_{8} \exp \left(\frac{-\left(t_{1}-p_{9}\right)^{2}}{2 p_{10}}\right)$

- For RR events two stages were required. Firstly a $t_{2}$ spectrum cut on a random $t_{1}$ region, with the same width as the prompt timing region (dashed blue line in fig. 5.19) was fitted with a 3rd degree polynomial background and a Gaussian(panel (iv)): $R R_{2}\left(t_{2}\right)=p_{1}+p_{2} t_{2}+p_{3} t_{2}^{2}+p_{4} t^{3}+$ $p_{5} \exp \left(\frac{-\left(t_{2}-p_{6}\right)^{2}}{2 p_{7}}\right)$. This parameterisation was then weighted with the result of a fit to the $t_{1}$, random-random spectrum $R R_{1}\left(t_{1}\right)$, panel (iii), which had the same form as $R P\left(t_{1}\right)$, to account for the random $t_{1}$ region used for the $t_{2}$ fit having a slightly reduced background compared to the prompt region.

Random $\phi_{n}^{\prime}$ distributions $R P_{i}, P R_{i}, R R_{i}{ }^{6}$ from events with time of flight outwith the prompt cuts, were created in parallel with the prompt data $P P_{i}$, to determine

\footnotetext{
${ }^{5} \mathrm{~A}$ Landau distribution is an asymmetric probability density function characterised by a narrow peak and a long tail to one side. The 3 parameters are analogous to those of a Gaussian, i.e area, most probable peak position and peak width.

${ }^{6} R P_{i}$ is a $\phi_{n}^{\prime}$ histogram collected from events with a random time in the first wall and prompt time in the second wall etc., $i$ refers to the bin number. For small bin width it tends to a continuous function of $\phi_{n}^{\prime}: R P_{i} \cong R P\left(\phi_{n}^{\prime}\right)$.
} 

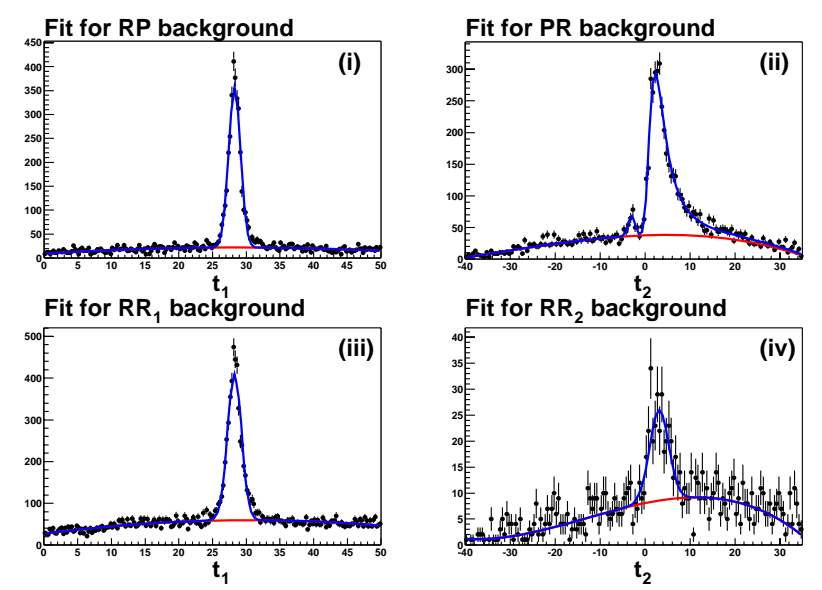

Figure 5.20: Fits to time-of-flight spectra in ns are shown with the blue line while the resulting contribution from the background is shown with the red line. The histogram of panel (i) is the projection of the region (b) fig. 5.19 on the $t_{1}$ axis. The histogram of panel (ii) is the projection of the region (c) on the $t_{2}$ axis. The histogram of panel (iii) is the projection of the region outwith (b) on the $t_{1}$ axis. The histogram of panel (iv) is the projection of the blue dashed region on the $t_{2}$ axis.

the shape of the random $\phi_{n}^{\prime}$ distributions. These histograms were then normalised using the results of the background fits :

$$
R_{i}^{\prime}=W_{R} R_{i} \text { with } W_{R}=\frac{\int_{t^{l o w}}^{t^{u p}} R(t) d t}{\sum_{i}^{i=n} R_{i}} \frac{1}{b_{R}}
$$

where $t^{u p}, t^{\text {low }}$ are the upper and lower prompt time limits, $b_{R}$ is the bin width of the time-of-flight histogram and $n$ is the number of bins. $R_{i}$ refers to one of $R P_{i}$, $P R_{i}$ or $R R_{i}$ and the integral is over either the first or second wall time-of-flight (fig. 5.20). The errors on the background distributions are discussed in Appendix A.1.

This procedure was applied independently to the four different combinations of helicity and $\phi_{n}^{\prime}$ range (eqn. 5.10). The three types of random distributions were then combined to give the overall background distribution $\Re_{i}$, and the random subtracted prompt neutron scattering distribution $N_{i}$ :

$$
\begin{gathered}
\Re_{i}=R P_{i}^{\prime}+P R_{i}^{\prime}-R R_{i}^{\prime} \\
N_{i}=P P_{i}-\Re_{i}
\end{gathered}
$$




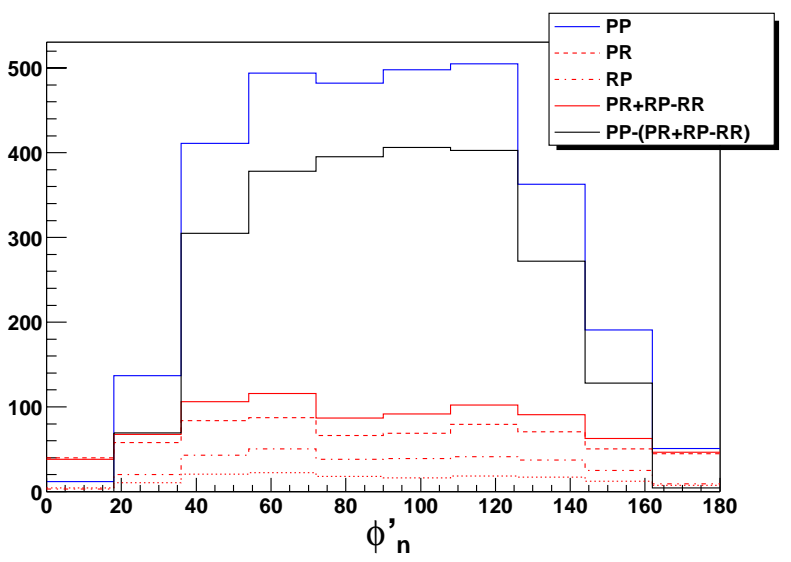

Figure 5.21: An example of distributions used for the random subtraction. The black line shows the resulting $\phi_{n}^{\prime}$ distribution used as part of the asymmetry calculation.

An example of the $\phi_{n}^{\prime}$ distributions from the four different prompt-random regions (PP, PR, RP, RR) and the resulting random subtracted distribution are shown in figure. The four $N_{i}$ histograms can now be combined as in eqn. 5.10 to give the asymmetry distribution.

By combining the four random histograms in a similar way the contribution of the background asymmetry $A_{\text {ran }}$ to the asymmetry with no random subtraction $A_{\text {all }}$ (eqn. 5.14), was determined, the results for each precession angle are shown in figure 5.22. These asymmetries of a few tenths of a percent give a small correction to the overall asymmetries which range typically from $\sim 2-10 \%$.

\subsection{Nuclear Structure Corrections}

Section 2.3 discussed the transfered polarisation of the electron to the recoil neutron $(\underline{P})$ in both free $n\left(\vec{e}, e^{\prime} \vec{n}\right)$ scattering and quasi-elastic $D\left(\vec{e}, e^{\prime} \vec{n}\right) p$ scattering. It was shown that for the Born approximation (one-photon exchange) and scattering on the quasi-elastic peak $\left(\theta_{n q}=0\right)$, the polarisation components are the same in both cases (i.e. eqn. 2.35). In the real experiment we sample data outwith both of these ideal cases and therefore corrections have to be made to account for these deviations. 


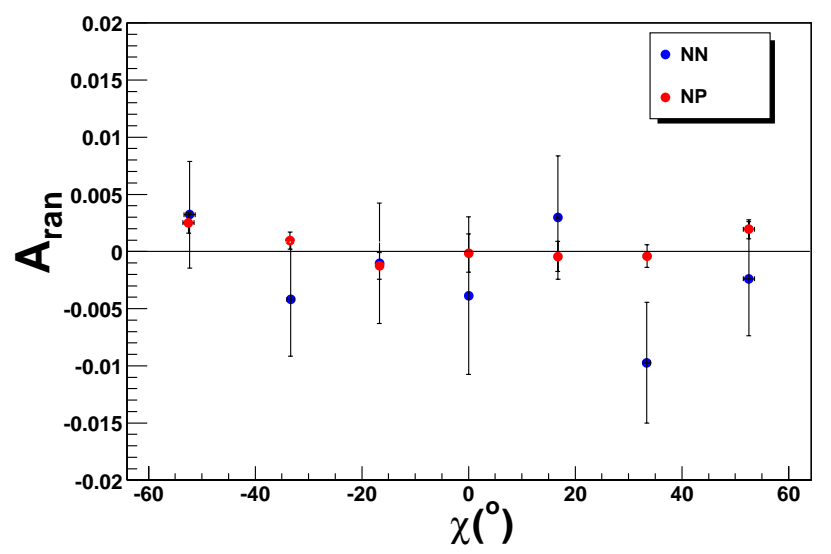

Figure 5.22: The contribution of the random background to the $A_{\text {all }}$ for both NN and NP type events.

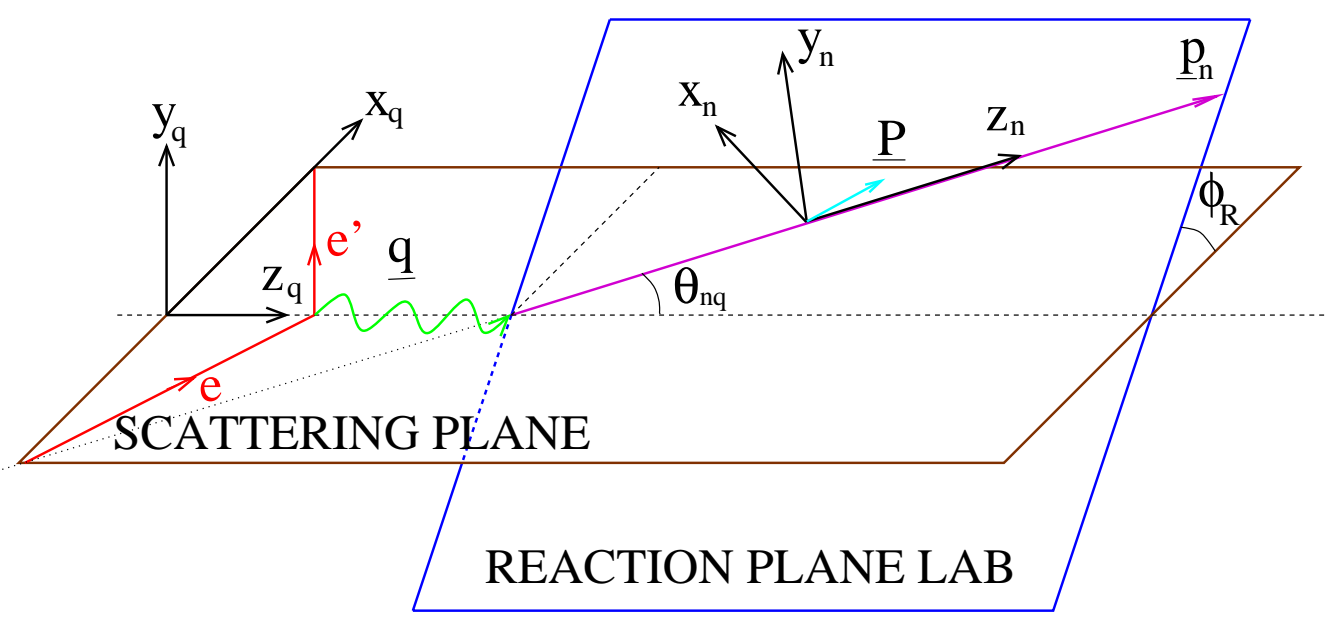

Figure 5.23: The neutron polarisation coordinate systems defined by the virtual photon $\left(x_{q}, y_{q}, z_{q}\right)$ and the neutron $\left(x_{n}, y_{n}, z_{n}\right)$. 


\subsubsection{Kinematic Correction}

Due to the large solid angles of the detectors, we also detected neutrons which had a significant initial Fermi motion $\underline{p}_{f}$. Therefore the recoil neutron momentum $\underline{p}_{n}$ may deviate from the direction of the virtual photon $q$ (fig. 5.23). The polarisation we measure in the polarimeter $\left(\underline{P}^{n}\right)$ is in a coordinate system with the z-axis along $\underline{P}_{n}$, whereas the polarisation components $\left(\underline{P}^{q}\right)$ that are related to the form factors through eqns. 3.1-3.3 are given in a coordinate system with the z-axis along the virtual photon momentum, $q$ :

$$
\begin{aligned}
\underline{z_{n}}=\underline{\widehat{p_{n}}} \quad \underline{y_{n}}=\underline{\widehat{p_{n}}} \times \underline{\widehat{q}} \quad \underline{x_{n}}=\underline{\widehat{y_{n}}} \times \underline{\widehat{z_{n}}} \\
\underline{z_{q}}=\underline{\widehat{q}} \quad \underline{y_{q}}=\underline{\widehat{q}} \times \underline{\widehat{p_{e}}} \quad \underline{x_{q}}=\underline{\widehat{y_{q}}} \times \underline{\widehat{z_{q}}}
\end{aligned}
$$

Therefore to calculate $G_{E}^{n}$ using eqn. 3.4 the neutron polarisation has to be rotated back into the photon frame using a Wigner rotation [20, 52] which accounts for the Lorentz boost between the two frames. The Wigner rotation from the photon frame to the neutron frame is :

$$
\begin{aligned}
\underline{P}^{n}= & D_{z}^{-1}\left(\phi_{R}\right) \cdot D_{y^{\prime}}\left(\theta_{W}\right) \cdot D_{z}\left(\phi_{R}\right) \cdot \underline{P}^{q} \\
= & \left(\begin{array}{ccc}
\cos \phi_{R} & -\sin \phi_{R} & 0 \\
\sin \phi_{R} & \cos \phi_{R} & 0 \\
0 & 0 & 1
\end{array}\right) \cdot\left(\begin{array}{llr}
\cos \theta_{W} & 0 & -\sin \theta_{W} \\
0 & 1 & 0 \\
\sin \theta_{W} & 0 & \cos \theta_{W}
\end{array}\right) \\
& \cdot\left(\begin{array}{lll}
\cos \phi_{R} & \sin \phi_{R} & 0 \\
-\sin \phi_{R} & \cos \phi_{R} & 0 \\
0 & 0 & 1
\end{array}\right) \cdot \underline{P}^{q} \\
= & \left(\begin{array}{lll}
1 & 0 & -\sin \theta_{W} \cos \phi_{R} \\
0 & & 1
\end{array}\right) \cdot \underline{P}^{q}+O\left(\theta_{W}^{2}\right)(5.18) \\
\sin \theta_{W} \cos \phi_{R} & \sin \theta_{W} \sin \phi_{R} \quad r \phi_{R}
\end{aligned}
$$

where $\phi_{R}$ is the angle between the reaction and scattering planes and $\theta_{W}$ is the Wigner angle [76]:

$$
\sin \theta_{W}=\frac{1+\gamma\left(p_{i}\right)}{\gamma(q)+\gamma\left(p_{n}\right)} \theta_{n q}
$$



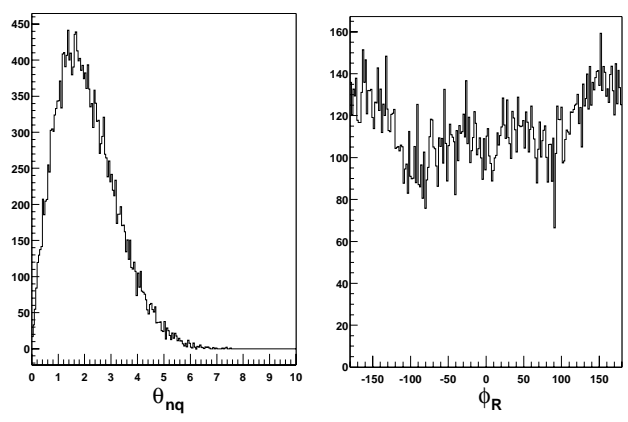

Figure 5.24: Distributions of the angles $\theta_{n q}$ and $\phi_{R}$ in degrees (fig. 5.23).
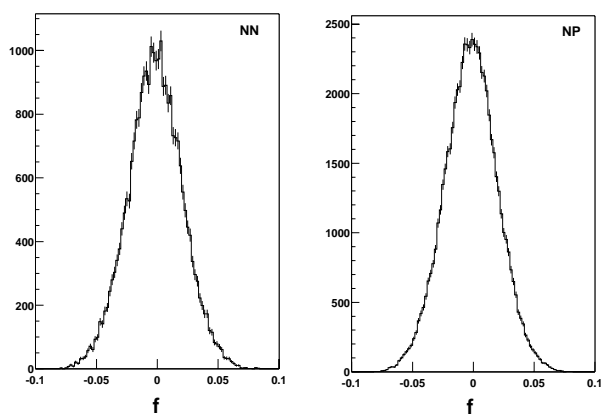

Figure 5.25: The distribution of factor $f$ for the whole data set NN and NP event.

and $\gamma(p)=\frac{E_{n}(p)}{m_{n}}$ are the boost parameters of the neutron with $p_{i}, q$, and $p_{n}$ being the momentum of the initial neutron, virtual photon and struck neutron respectively, and $\theta_{n q}$ is the angle between the recoil neutron and virtual photon. The spin precession technique measures the mean polarisation ratio $\tan \chi_{\circ}=\frac{P_{x}^{n}}{P_{z}^{n}}$ of the analysed neutron events (sec. 4.5.2). Using equation 5.18 to relate this to the mean ratio in the photon frame:

$$
\frac{P_{x}^{q}}{P_{z}^{q}}=\frac{\bar{f}+\frac{P_{x}^{n}}{P_{z}^{n}}}{1-\bar{f} \frac{P_{x}^{n}}{P_{z}^{n}}}=\frac{P_{x}^{n}}{P_{z}^{n}}+\bar{f}\left(1+\left(\frac{P_{x}^{n}}{P_{z}^{n}}\right)^{2}\right)+O\left(\bar{f}^{2}\right)
$$

where $\bar{f}=\overline{\sin \theta_{W} \cos \phi_{R}}$. If the polarimeter acceptance is symmetric around its z-axis then $\overline{\sin \theta_{W} \cos \phi_{R}}=0$, the effect of the Fermi motion cancels and no correction is required. However if there is a slight asymmetry in the polarimeter acceptance $\overline{\sin \theta_{W} \cos \phi_{R}} \neq 0$, this has to be corrected for. Figure 5.24 shows an example of the experimental $\theta_{n q}$ and $\phi_{R}$ distributions. Figure 5.25 shows the resulting $f$ distributions for NN and NP events with the means of these distributions giving $\bar{f}=-0.0020 \pm 0.0001$ for both NN and NP events. 


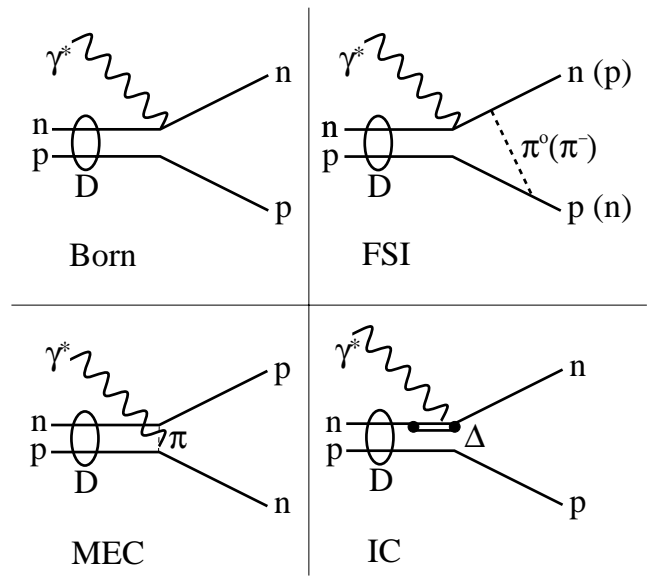

Figure 5.26: The different contributions to quasi-elastic electron scattering. Born : one-photon exchange. FSI : final state interaction, an exchange of a meson between the nucleons after the electron has scattered. MEC : the photon couples to a meson being exchanged by the nucleons. IC : excitation of the nucleon to an intermediate $\Delta$ resonance state.

\subsubsection{Beyond Plane-Wave Impulse Approximation}

The Plane-Wave Impulse Approximation (PWIA) ignores contributions to the electron-neutron scattering reaction from final state interactions(FSI), meson exchange currents (MEC) and isobaric currents (IC) (fig. 5.26). In addition relativistic corrections have to be made to a non-relativistic formalism. These contributions have been included in a model by Arenhövel et al $[28]^{7}$, which has been used to calculate the polarisation components as a function of scattered electron energy $\left(E_{e}^{\prime}\right)$ and angle $\left(\theta_{e}\right)$ and also the angle between the recoil neutron and the virtual photon $\theta_{n q}(F u l l)$. In addition the polarisation was calculated assuming the Plane Wave Born Approximation with relativistic corrections(Born), thus allowing the deviations due to nuclear structure to be determined. The measured polarisation was then corrected by this deviation to allow the polarisation ratio to be expressed in terms of the electromagnetic form factors as in equation 3.4.

$$
\left(\frac{P_{x}}{P_{z}}\right)=\left(\frac{P_{x}}{P_{z}}\right)^{\text {meas }}-\left[\left(\frac{P_{x}}{P_{z}}\right)^{\text {Full }}-\left(\frac{P_{x}}{P_{z}}\right)^{\text {Born }}\right]=\tan \chi_{\circ}-\Delta R_{P}
$$

\footnotetext{
${ }^{7}$ In this model the proton and neutron magnetic form factors are assumed to have Dipole form and the Galster parameterisation is used for the neutron electric form factor. The Bonn r-space potential is used for the nucleon-nucleon potential.
} 

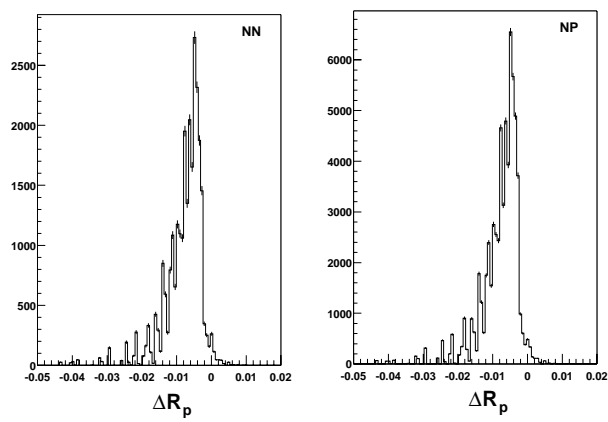

Figure 5.27: Distribution of $\Delta R_{P}$, to correct for FSI, MEC and IC effects (fig. $5.26)$

For each event the Full and Born polarisation components are found in a precompiled lookup table which consists of five bins each for $E_{e}^{\prime}, \theta_{e}$ and 15 bins for $\theta_{n q}$ [52]. This allows $\Delta R_{P}$ to be estimated for each event and the distribution for the full data set is shown in figure 5.27. The correction to the measured polarisation is taken as the mean of this distribution giving $\Delta R_{P}=-0.0080 \pm 0.0003$ for NN and $\Delta R_{P}=-0.0078 \pm 0.0003$ for NP. 


\section{Chapter 6}

\section{Results and Discussion}

\subsection{Asymmetry versus Precession angle}

\subsubsection{Fitting the spin precession}

The previous section 5 described how to derive from the raw experimental data an asymmetry and spin precession angle for each of the seven magnetic field settings. The next stage is to find the precession angle where the asymmetry crosses zero $\chi_{\circ}$ and thus the ratio of the transverse $\left(P_{x}^{n}\right.$, as $\left.P_{y}^{n} \simeq 0\right)$ to longitudinal $\left(P_{z}^{n}\right)$ components of neutron polarisation in the frame defined in equation 5.16, by using equation $\frac{P_{x}^{n}}{P_{z}^{n}}=\tan \chi_{\circ}$.

For a particular magnetic field setting, with an associated spin precession of $\chi$, the asymmetry $A(\chi)$ is directly proportional to $P_{x}^{n}(\chi)$, the transverse spin after a precession of $\chi$. After its spin is precessed the neutron's transverse polarisation is given by :

$$
P_{x}(\chi)=P_{n} \sin \left(\chi-\chi_{\circ}\right)
$$

where $P_{n}$ is the magnitude of the neutron polarisation and $\chi_{\circ}$ is the angle between the polarisation before spin precession and the initial neutron momentum direction. Substituting this into equation 5.12, gives the asymmetry as a function of $\chi$ :

$$
A(\chi)=\alpha_{e f f} P_{e} P_{n} \sin \left(\chi-\chi_{\circ}\right)=p 0 \cdot \sin (\chi+p 1)
$$

A two parameter sinusoidal fit to the $A(\chi)$ data points determines both $\chi_{\circ}=-p 1$ 


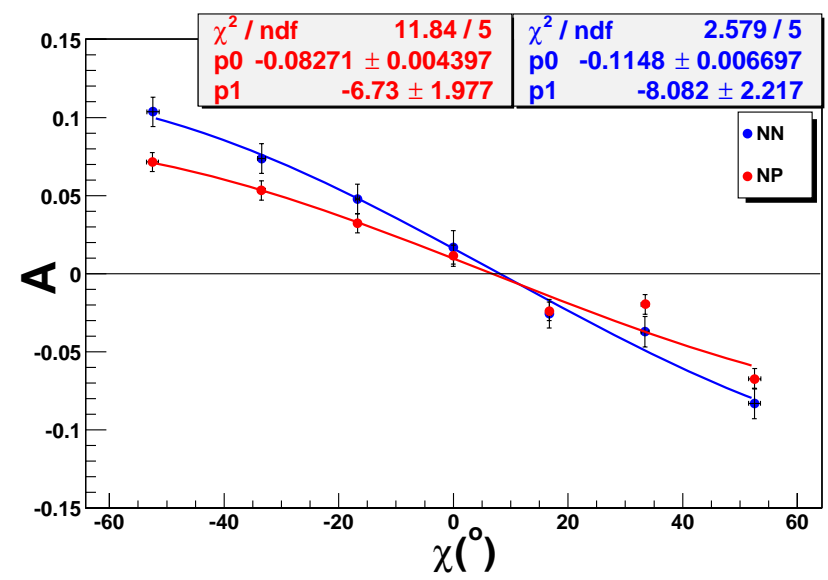

Figure 6.1: Asymmetry versus precession angle for both NN(blue) and NP(red) type events. The boxes show the fit results for each case.

and the product $\alpha_{e f f} P_{e} P_{n}=|p 0|$. The fit procedure used the ROOT utility TMinuit, which essentially calls the CERN package MINUIT [77] to perform a non-linear least squares fit. Plots of $A(\chi)$ with the resulting fits are shown in figure 6.1 for both NN and NP data. Also shown are the fit parameters with their associated errors and the fit $\chi^{2}$, with $n d f$ the number of degrees of freedom for the fit. The parameter $p 1$, i.e. $\chi_{\circ}$ which leads to $G_{E}^{n}$ is independent of $\alpha_{\text {eff }}$. However if $\alpha_{\text {eff }}$ and therefore the amplitude of the sinusoid is increased, the steeper gradient at $A(\chi)=0$ will constrain $p 1$ more, leading to a smaller error in $G_{E}^{n}$.

To obtain the effective analysing power $\alpha_{\text {eff }}$, one must know the electron beam polarisation $P_{e}$ and $P_{n}$, which is the polarisation transferred to the neutron by an electron beam with $P_{e}=1$. The beam polarisation was monitored by a Møller polarimeter (sec. 4.2), and for the 2001, $0.8\left(\frac{\mathrm{GeV}}{c}\right)^{2}$ production runs this was $P_{e}=84 \pm 3 \%$ (fig. 4.3). To estimate the transferred polarisation for the purpose of calculating $\alpha_{\text {eff }}$, one can use the formula of Arnold, Carlson and Gross (eqns. 3.1-3.3) with the Galster parameterisation for $G_{E}^{n}$ and the dipole parameterisation for $G_{M}^{n}$. This results in $P_{x}=0.133, P_{z}=0.95$ and $P_{n}=\sqrt{P_{x}^{2}+P_{z}^{2}}=0.96$, which yields $\alpha_{e f f}=\frac{|p 0|}{0.81}$.

The results are summarised in the table below. 


\begin{tabular}{|c|c|c|}
\hline & $\mathrm{NN}$ & $\mathrm{NP}$ \\
\hline \hline$\chi_{\circ}\left(^{\circ}\right)$ & $8.08 \pm 2.22$ & $6.73 \pm 1.98$ \\
\hline$\frac{P_{x}^{n}}{P_{z}^{n}}$ & $0.141 \pm 0.039$ & $0.118 \pm 0.035$ \\
\hline$\alpha_{e f f} P_{e} P_{n}$ & $0.115 \pm 0.007$ & $0.083 \pm 0.004$ \\
\hline$\alpha_{e f f}$ & $0.142 \pm .009$ & $0.102 \pm 0.005$ \\
\hline
\end{tabular}

\subsubsection{Effect of Random Subtraction}

The random subtraction procedure is outlined in section 5.5.1. Figures 6.2 and 6.3 show the asymmetries for each precession angle with and without random subtraction, for both NN and NP events. For NN events the amplitude of the sine fit is increased by about $25 \%$ while for NP events the increase is only around a few percent. For NN events the random background mainly comes from gamma radiation and as a result is largely independent of precession magnet field. As the neutron detector is set at a forward angle with respect to the electron beamline the number of these background events is quite high and therefore the shift in the asymmetries is relatively large. For NP events, where a further coincidence is required with a veto detector in the second wall, the probability of a random triple coincidence from gamma interactions is very small. The contribution to the background from charged particles interacting in both a veto detector and scintillator bar in the second wall is more significant and highly dependent on the magnetic field, leading to a different correction for each precession angle. The number of these events is still small and so the difference to the distribution with and without random subtraction is slight. Table 6.1 gives the measured values of asymmetries, crossing points, the real-to-random ratio and the number of events analysed for each field setting. Table 6.2 shows the resulting values of $\alpha_{\text {eff }}$ and $\frac{P_{x}^{n}}{P_{z}^{n}}$ derived from the sinusoidal fit parameters.

\subsection{Proton Misidentification}

A possible source of false asymmetry, leading to a systematic error in the crossing point $\chi_{\circ}$, is the misidentification of protons as neutrons in the first wall of the polarimeter, due to counting losses in the first wall veto detectors. This can create a false asymmetry in a number of ways:

1. The proton polarisation vector will in general point in a different direction to that of the neutron, as can be seen from equations 3.1-3.3, which would 


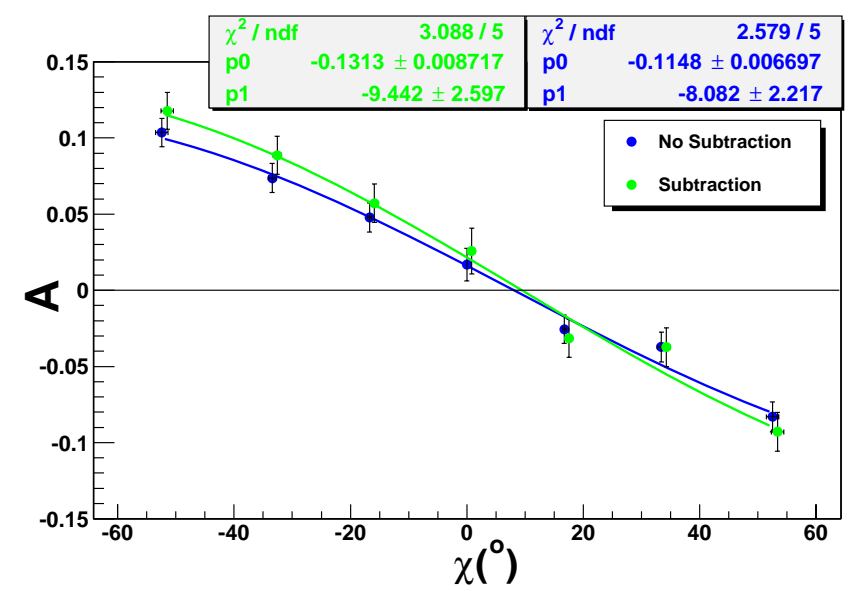

Figure 6.2: $A_{\text {real }}$ (asymmetries with background subtraction-green) and $A_{\text {all }}$ (asymmetries without background subtraction-blue) for NN events. Also shown are the resulting sinusoidal fits and parameters.

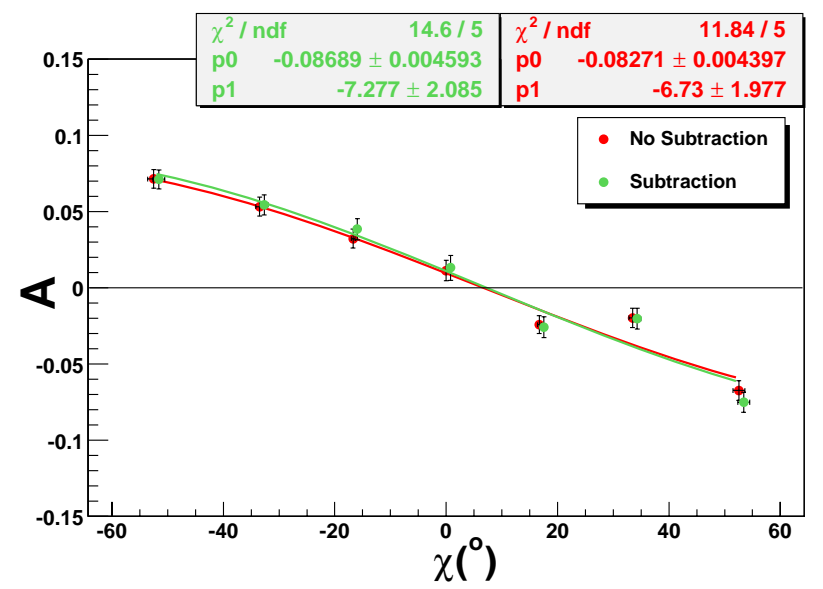

Figure 6.3: $A_{\text {real }}$ (green) and $A_{\text {all }}$ (red) for NP events. Also shown are the resulting sinusoidal fits and parameters. 


\begin{tabular}{|c|c|c|c|c|c|}
\hline $\mathrm{NN}$ & $\chi\left(^{\circ}\right)$ & $A_{\text {all }}$ & $r$ & $\mathrm{~N}$ & $A_{\text {real }}$ \\
\hline \hline+400 & $52.52 \pm 1.05$ & $-0.083 \pm 0.010$ & $5.13 \pm 0.18$ & 12180 & $-0.093 \pm 0.013$ \\
\hline+250 & $33.43 \pm 0.67$ & $-0.037 \pm 0.010$ & $5.10 \pm 0.17$ & 12108 & $-0.037 \pm 0.013$ \\
\hline+125 & $16.72 \pm 0.33$ & $-0.026 \pm 0.009$ & $4.77 \pm 0.15$ & 13244 & $-0.032 \pm 0.013$ \\
\hline+000 & $0 \pm 0$ & $0.017 \pm 0.011$ & $4.3 \pm 0.15$ & 9704 & $0.026 \pm 0.015$ \\
\hline-125 & $-16.72 \pm 0.33$ & $0.048 \pm 0.010$ & $5.03 \pm 0.17$ & 12830 & $0.057 \pm 0.013$ \\
\hline-250 & $-33.46 \pm 0.67$ & $0.074 \pm 0.010$ & $5.22 \pm 1.78$ & 12686 & $0.089 \pm 0.012$ \\
\hline-400 & $-52.41 \pm 1.05$ & $0.104 \pm 0.010$ & $5.22 \pm 1.68$ & 13086 & $0.118 \pm 0.012$ \\
\hline \hline $\mathrm{NP}$ & $\chi\left(^{\circ}\right)$ & $A_{\text {all }}$ & $r$ & $\mathrm{~N}$ & $A_{\text {real }}$ \\
\hline \hline+400 & $52.56 \pm 1.05$ & $-0.067 \pm 0.006$ & $42.19 \pm 5.8$ & 32864 & $-0.074 \pm 0.007$ \\
\hline+250 & $33.46 \pm 0.67$ & $-0.020 \pm 0.006$ & $24.63 \pm 1.96$ & 33820 & $-0.019 \pm 0.007$ \\
\hline+125 & $16.72 \pm 0.33$ & $-0.024 \pm 0.006$ & $11.48 \pm 0.48$ & 38046 & $-0.025 \pm 0.007$ \\
\hline+000 & $0 \pm 0$ & $0.011 \pm 0.007$ & $8.08 \pm 0.32$ & 27276 & $0.014 \pm 0.008$ \\
\hline-125 & $-16.72 \pm 0.33$ & $0.032 \pm 0.006$ & $11.43 \pm 0.54$ & 34730 & $0.039 \pm 0.007$ \\
\hline-250 & $-33.48 \pm 0.67$ & $0.053 \pm 0.006$ & $28.64 \pm 2.88$ & 35124 & $0.055 \pm 0.006$ \\
\hline-400 & $-52.51 \pm 1.05$ & $0.071 \pm 0.006$ & $52.89 \pm 3.67$ & 36356 & $0.072 \pm 0.006$ \\
\hline
\end{tabular}

Table 6.1: The above tables summarise the calculated asymmetries, precession angles, number of real events and the real-to-random ratio of plots 6.2 and 6.3. $A_{\text {all }}$ is without random subtraction, $A_{\text {real }}$ is with random subtraction.

\begin{tabular}{|c|c|c|}
\hline & NN & NP \\
\hline \hline$\chi_{\circ}\left({ }^{\circ}\right)$ & $9.44 \pm 2.60$ & $7.28 \pm 2.10$ \\
\hline$\frac{P_{x}^{n n}}{P_{z}^{n}}$ & $0.166 \pm 0.046$ & $0.128 \pm 0.037$ \\
\hline$\alpha_{e f f} P_{e} P_{n}$ & $0.131 \pm 0.009$ & $0.087 \pm 0.005$ \\
\hline$\alpha_{e f f}$ & $0.162 \pm .011$ & $0.107 \pm 0.006$ \\
\hline
\end{tabular}

Table 6.2: Polarisation ratio and $\alpha_{\text {eff }}$ derived from the sinusoidal fit parameters, with random background subtracted. 

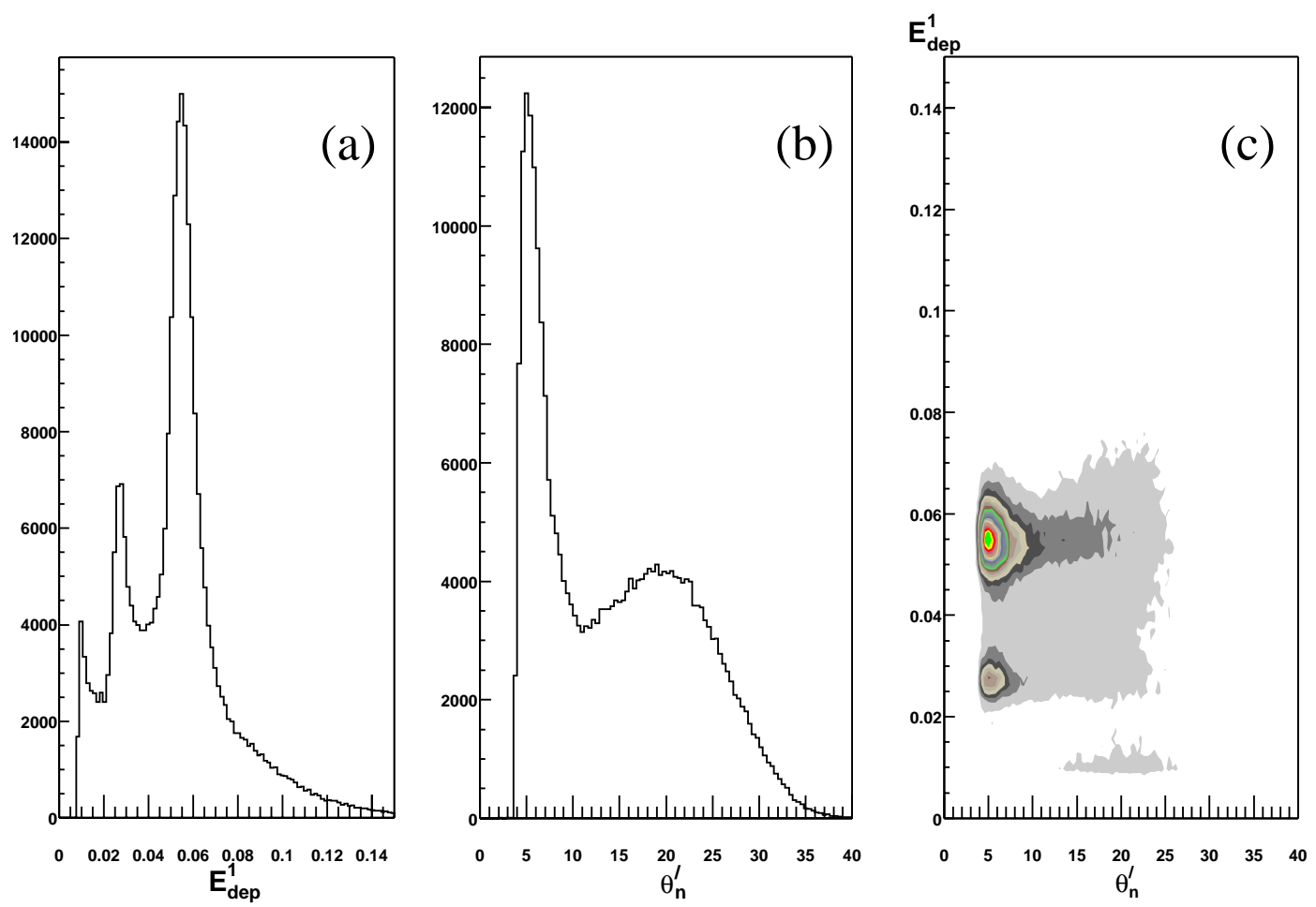

Figure 6.4: Energy deposited in the first wall, $E_{\text {dep }}^{1}(\mathrm{GeV})$ and scattering angle $\theta_{n}^{\prime}$ (degrees) spectra for events with a veto coincidence in both the first and second polarimeter walls i.e protons.

result in a phase shift of the $A(\chi)$ distribution.

2. The application of a magnetic field along the flight path of the proton causes a bending of its trajectory, as well as precessing its spin, so the precession relative to the momentum direction is no longer described by equation 4.5.

3. Due to the bending, the number of protons incident on the polarimeter is precession-field dependent, making the relative number of protons contaminating the sample precession-angle dependent.

\subsubsection{Proton signals}

To investigate the effect of protons from the $\mathrm{LH}_{2}$-target on the measured crossing point it is instructive to look at a sample of data where first wall hits are in coincidence with a veto detector, (i.e. charged protons) to localise the kinematic regions where protons are observed. 

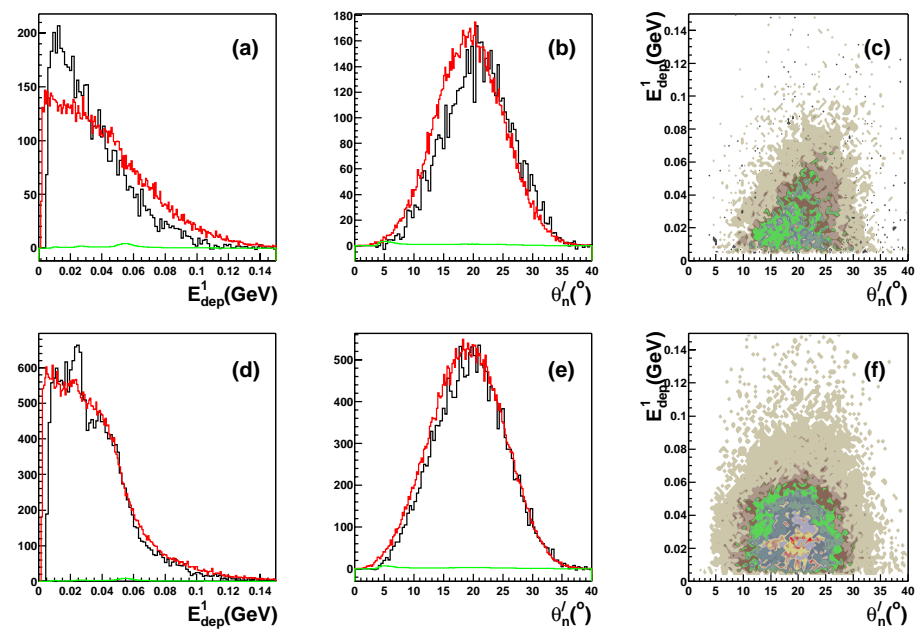

Figure 6.5: The top 3 panels(a-c) show NN events, the bottom 3 (d-f) NP events, with the black line from experiment and red line from simulation.(a,d) are the energy deposited in the first scintillator wall, $(b, e)$ the neutron scattering angle between first and second walls and (c,f) the corresponding two-dimensional plots.

The clearest signals of protons are in the energies deposited in the first wall $\left(E_{d e p}^{1}\right)$ and the scattering angles between first and second walls $\left(\theta_{n}^{\prime}\right)$. These are shown in figure 6.4. For the $E_{d e p}^{1}$ spectra the strong peak at $0.05 \mathrm{GeV}$ is from protons recording hits in both layers of the first wall, while the weaker peak at $0.025 \mathrm{GeV}$ results from occasions where a hit in one of the layers has not been recognised. This could be due to missing the timing cut or an ADC or TDC entry. For the $\theta_{n}^{\prime}$ spectra the large peak at small angles is associated with protons that interact with atomic electrons only in the lead wall and the first scintillator wall. The bump at larger angles contains protons that have undergone additional nuclear interactions and consequently has a similar shape to the neutron $\theta_{n}^{\prime}$ distribution. The two dimensional plot clearly shows the peaks in the one-dimensional plots are related, with the protons leaving two distinct clusters which can be used as a marker for where proton contamination will occur in neutron spectra. Even though protons not undergoing a nuclear reaction will not show a scattering asymmetry, their presence can be used to estimate the number of protons which do, as misidentification by the veto detectors should be independent of this. 

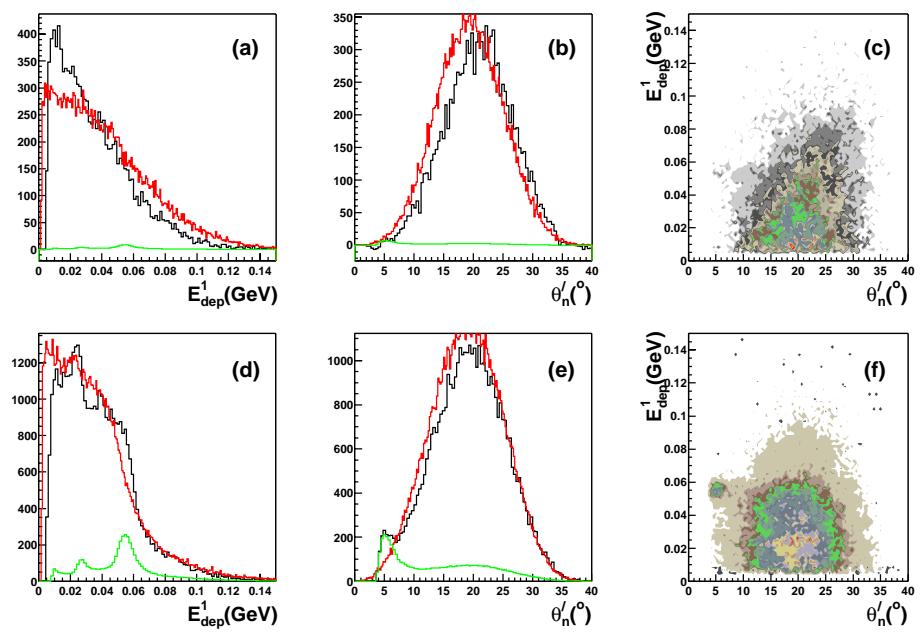

Figure 6.6: As for fig. 6.5, but the experiment data now have deliberate proton contamination.

\subsubsection{Neutron Signals}

In the case of neutrons scattering in the first wall, the characteristics of $E_{d e p}^{1}$ and $\theta_{n}^{\prime}$ must arise solely from strong interaction scattering. Figure 6.5, shows the $E_{d e p}^{1}$ and $\theta_{n}^{\prime}$ spectra. The black lines show the data, the red lines show the predictions of the simulation (sec. 5.1), and the green lines are explained below. The 2-d plots show the data only. The top three plots are for the case of detecting a neutron in the second wall $(\mathrm{NN})$ while the bottom three plots relate to a proton in the second wall(NP). In figure 6.5:(c) showing $E_{d e p}^{1} v s \theta_{n}^{\prime}$, for the case of neutrons scattering into the second wall a hint of the linear relation between the scattering angle and energy deposited can be observed, which is just the relation $T_{p}^{\prime} \propto \sin ^{2} \theta_{n}^{\prime}$ discussed in section 6.3.2. The peaks associated with protons in the first wall (fig. 6.4:(a,b)) are missing from these spectra (fig. 6.5), which confirms that neutron identification in the first wall is good. Note, the bump (and subsequent dip) in the NP $E_{\text {dep }}^{1}$ spectrum at $\sim 0.02 \mathrm{GeV}$, (fig. 6.5:(d)), is due to interactions occurring at the back of the first scintillator layer so that the proton does not travel through enough of the scintillator to pass the hardware threshold of $\sim 4$ $\mathrm{MeV}$.

To test our confidence that there is no significant proton contamination of the neutron data, we have artificially misidentified some protons as neutrons and investigated the effect on the spectra, as shown in figure 6.6. We now observe a clear bump in the NP $\theta_{n}^{\prime}$ plot at $5^{\circ}$, associated with the protons which do not 
undergo nuclear scattering. There is also a small hint of this in the NN data but less pronounced as we still demand that there is no veto coincidence in the second wall. The green lines show the proton spectra from figure 6.4 scaled to the non-nuclear scattering $\theta_{n}^{\prime}$ peak (fig. 6.6:(e)). Applying the same scaling to the $E_{d e p}^{1}$ spectra (fig. 6.6:(d)), we see that the protons are responsible for the slight divergence of the data from the simulation at $E_{\text {dep }}^{1} \sim 0.05 \mathrm{GeV}$, which is not present in figure 6.5:(d).

By comparing the central scattering angle region we can deduce that the protons have contaminated the neutron signal by $<1 \%$ for $\mathrm{NN}$ and $<5 \%$ for NP, in this artificial case. For the real neutron samples of figure 6.5 the proton contamination is below $1 \%$ for NP events and below $0.5 \%$ for NN events. Indeed, figure 6.5 relates to the case of no precession magnetic field and when the field is applied the contamination is further reduced.

\subsection{Comparison of Simulated and Real Data}

The data from both the real experiment and simulations have been analysed and compared in order to test the predictions of the Monte Carlo model of the polarimeter, thereby assessing our understanding of the polarimeter set-up. In particular, in the simulation it was hypothesised that quasi-elastic scattering on carbon is the dominant reaction channel and that the analysing power for this was similar to that of free nucleon-nucleon scattering. Also we wanted to determine if there was a significant contribution from charge exchange scattering, resulting in protons detected in the second wall with a similar analysing power as the neutrons detected with no charge exchange. Neither of these possibilities had been explicitly tested in previous recoil polarimetry measurements.

For a given kinematic variable two comparisons were made: firstly on the distribution of that variable and secondly on the dependence of the effective analysing power on that variable. The crossing point $\chi_{\circ}$, should not vary in any systematic way with any kinematic variable, apart from those associated with the bound neutron corrections, as it is in all cases determined by the incident neutron spin direction relative to its momentum.

In the following, events where either neutrons(NN) or protons(NP) have been detected in the second wall of the polarimeter are separated. The two cases have different analysing powers (fig. 4.10) and also some kinematic variables have different meanings for the two cases. The NN events have a higher effective 

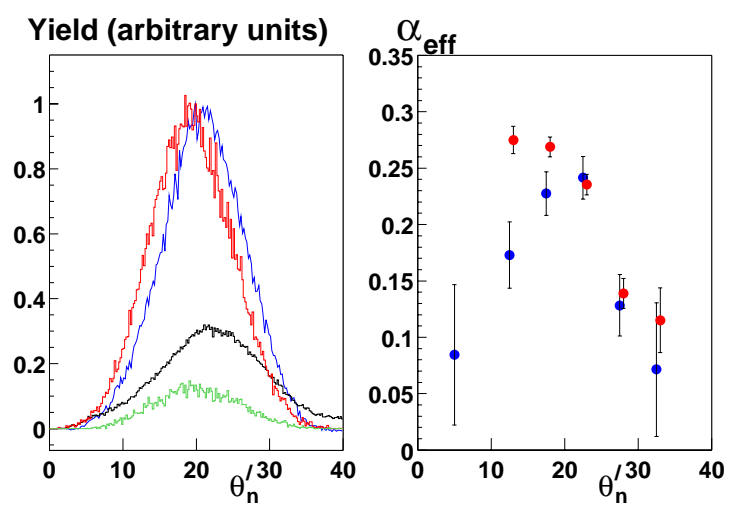

Figure 6.7: NN results for $\theta_{n}^{\prime}$. The left hand plot shows the distribution for the all simulated events(red line), simulated $p\left(n, n^{\prime}\right) p^{\prime}$ events (green), real events(blue) and the random background(black line). The right hand plot shows the effective analysing power from the simulation(red points) and data(blue points).

analysing power, while the NP events have more counts and a better real-torandom background ratio.

\subsection{1 $\theta_{n}^{\prime}$}

The scattering angle of the neutron in the first wall of the polarimeter can be accurately determined, since the walls of highly segmented scintillator give an accurate hit-position reconstruction. For neutron elastic scattering from Hydrogen the lab. angle is related unambiguously to the centre of mass angle $\theta_{C M}$ and thus the analysing power, (fig. 4.10). For quasi-elastic n-p scattering, as assumed in the simple model of $n+{ }^{12} C$ interactions (sec. 5.1.3), this relationship will be smeared out due to the effects of nucleon fermi motion. At an incident neutron momentum of $\sim 980\left(\frac{\mathrm{MeV}}{c}\right)$, obtained at $Q^{2}=0.8\left(\frac{\mathrm{GeV}}{\mathrm{c}}\right)^{2}$, this smearing is not small as the typical Fermi momentum in carbon is $\sim 220\left(\frac{\mathrm{MeV}}{\mathrm{c}}\right)$. The cross-section for the quasi-elastic channels is an order of magnitude larger than for scattering on hydrogen and therefore the former channel provides the dominant contribution to both the kinematic distributions and the effective analysing power. The comparison of simulation and data should therefore show if the simple n-p quasi-elastic scattering model is a reasonable assumption.

The results for NN and NP events are shown in figures 6.7 and 6.8 respectively and are discussed in the following. 

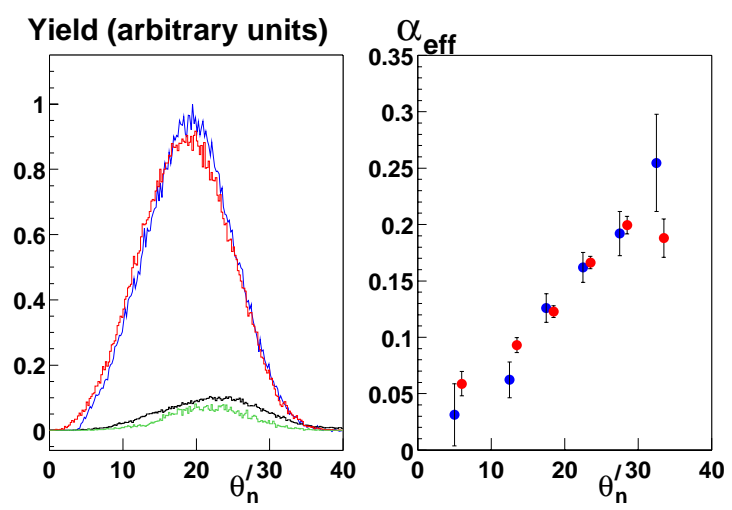

Figure 6.8: NP results for $\theta_{n}^{\prime}$. The left hand plot shows the distribution for the all simulated events(red line), simulated $p\left(n, p^{\prime}\right) n^{\prime}$ events (green), real events(blue) and the random background(black line). The right hand plot shows the effective analysing power from the simulation(red points) and data(blue points).

\section{Yields as a function of $\theta_{n}^{\prime}$}

For NN events (fig. 6.7), the simulated $\theta_{n}^{\prime}$ distribution is shifted slightly to small angles. This is largely caused by NP events, which pass under the second wall veto detectors and hit the bottom of the neutron detectors, being thus analysed as NN events. These events occur both in the simulation and the real data. However, their hit position in the simulation is known exactly, whereas in the real data the vertical coordinate of the horizontally aligned bar is unknown and thus the middle y and $\mathrm{z}$ coordinates of the bar are taken (sec. 5.2.4). This corresponds to a shift in hit position of greater than $10 \mathrm{~cm}$ (half the vertical dimension of the bar) and in $\theta_{n}^{\prime}$ of greater than $1.7^{\circ}$, for a $3 \mathrm{~m}$ separation of the first and second walls. Apart from this special case, any shift in the $\theta_{n}^{\prime}$ distribution resulting from taking middle coordinates as the hit position cancels as the angular variation is relatively slow, giving a similar number of events above and below the middle vertical point. The measured $\theta_{n}^{\prime}$ does not enter in the calculation of the asymmetries and so the data does not need to be corrected for this effect.

For NP events (fig. 6.8), there is not a corresponding problem and the two distributions are almost identical.

\section{$\alpha_{e f f}$ as a function of $\theta_{n}^{\prime}$}

For both NN and NP events the simulated $\theta_{n}^{\prime}$ dependence of $\alpha_{\text {eff }}$ is consistent with the analysing power angular dependence of figure 4.10 , bearing in mind the 

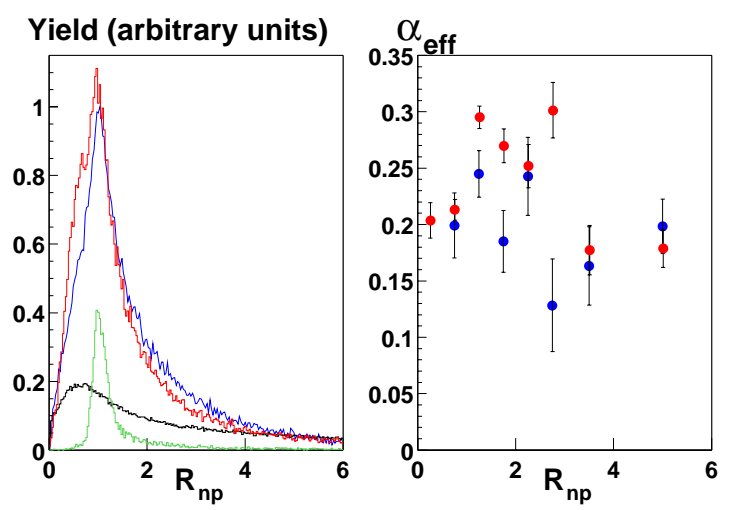

Figure 6.9: NN results for $R_{n p}$. The left hand plot shows the distribution for the all simulated events(red line), simulated $p\left(n, n^{\prime}\right) p^{\prime}$ events (green), real events(blue) and the random background(black line). The right hand plot shows the effective analysing power from the simulation(red points) and data(blue points)

polarimeter acceptance. For NN events that is approximately $\theta_{n}^{\prime}\left(10 \rightarrow 30^{\circ}\right) \equiv$ $\theta_{C M}\left(20 \rightarrow 60^{\circ}\right)$ and for NP events $\theta_{n}^{\prime}\left(10 \rightarrow 30^{\circ}\right) \equiv \theta_{C M}\left(160 \rightarrow 120^{\circ}\right)$. For NP events the agreement with the real data is particularly good, which is perhaps surprising considering the simple nature of the quasi-elastic model. The implication is that the quasi-elastic events do indeed have a similar analysing power to free n-p scattering. For NN events, the simulation does not match the data quite as well, although in the high acceptance region $\left(15-30^{\circ}\right)$ it is still a reasonable agreement. These results show the signal from NP scattering events is better understood than for NN, this is probably due to the detection of protons in the second wall being much "cleaner" than the detection of neutrons.

\subsubsection{Kinematic Variable $R_{n p}$}

When considering elastic scattering on hydrogen we can calculate the energy of the recoiling proton $T_{p}^{\prime}$ using two methods that are independent of time of flight measurements. The first is from the energy deposited in the scintillator, the second from the scattering angle $\theta_{n}^{\prime}$ and the incident neutron energy $T_{n}$ reconstructed from the photon information and neutron hit position, i.e :

$$
\begin{aligned}
T_{p}^{\prime}=E_{d e p}^{1} & =T_{n} \sin ^{2} \theta_{n}^{\prime} \\
R_{n p}=\frac{T_{n} \sin ^{2} \theta_{n}^{\prime}}{E_{d e p}^{1}} & =1 \text { (elastic scattering) }
\end{aligned}
$$


Forming a ratio of these gives a dimensionless variable $R_{n p}$, that is $\sim 1$ for the case of elastic NN scattering on hydrogen, shifted slightly due to the non-linear light response of the scintillator. Additionally, the recoiling proton will not be stopped in the scintillator in some cases, resulting in $E_{\text {dep }}^{1}$ being less than the full $T_{p}^{\prime}$ equivalent.

Using this variable allows the contribution of elastic neutron-hydrogen scattering to be emphasised.

For NP-type events this relation will no longer hold as $E_{d e p}^{1}$ is effectively a measure of the path length of the proton in the scintillator, which is just dependent on where in the scintillator the charge exchange interaction occured. Therefore, events occurring at the front of the first wall will have small $R_{n p}$ values and those at the back will have large $R_{n p}$.

\section{Yields as a function of $R_{n p}$}

The NN distribution (fig. 6.9) has two main components. The first is from elastic scattering events at $R_{n p}=1$, and their simulated contribution is shown by the green line. The second is from the $n+{ }^{12} C$, quasi-elastic events, where the $R_{n p}$ correlation will no longer apply due to the struck nucleon's initial Fermi momentum, giving these events a broadened distribution. A quasi-elastic shoulder is just visible in the real data and more clearly in the simulated data at $R_{n p} \simeq 0.6$. That it is smaller in the real experimental data implies the quasi-elastic crosssection may be overestimated relative to the elastic scattering, in the simulation. Most of the width of the elastic scattering peak is due to the non-linear light response (sec. 5.1.4), of the scintillator.

For the NP events (fig. 6.10) the experimental and simulated yield distributions again agree well.

\section{$\alpha_{e f f}$ as a function of $R_{n p}$}

The NN analysing power for simulated and real data shows a large degree of scatter so that it is difficult to draw any firm conclusions as to the systematic behaviour. Around $R_{n p} \simeq 2.5$ the real data does not show the pronounced "maximum" of the simulation. The reason for this structure in the simulated data is the large number of small $\theta_{C M}$, and therefore large $\alpha_{\text {eff }}$ events generated by the model in this $R_{n p}$ region. Two possibilities as to why the simulation does not reproduce the data are, 

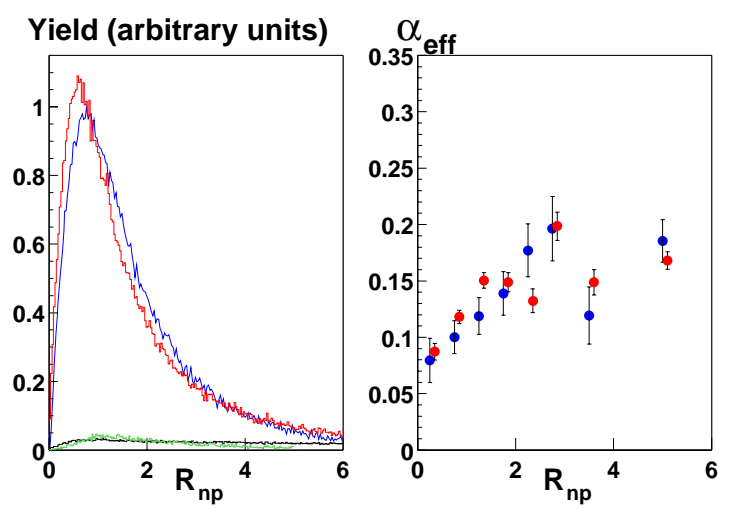

Figure 6.10: NP results for $R_{n p}$ with the same key as for 6.8. The left hand plot shows the distribution for the all simulated events(red line), simulated $p\left(n, p^{\prime}\right) n^{\prime}$ events (green), real events(blue) and the random background(black line). The right hand plot shows the effective analysing power from the simulation(red points) and data(blue points).

1. The relation of $R_{n p}$ to $\theta_{C M}$ resulting from the simple model is not correct.

2. The relationship between analysing power and $\theta_{C M}$ does not hold exactly for quasi-elastic scattering.

This is consistent with the low $\alpha_{\text {eff }}$ of the real data for small $\theta_{n}^{\prime}$ seen in figure 6.7 .

The NP analysing power increases for both the data and simulation which is expected, since $\alpha_{\text {eff }}$ will not vary with $E_{d e p}^{1}$, which is now only a measure of the path length of the proton in the first wall. The variation then arises mainly from the $\sin ^{2} \theta_{n}^{\prime}$ dependence, as is seen from figure 6.8 where $\alpha_{e f f}$ rises with increasing $\theta_{n}^{\prime}$.

\subsubsection{Energy loss of the projectile}

The energy loss of the projectile nucleon in the first wall scattering process, $\Omega$, can also be used to investigate the quasi-elastic model. Similar work has been done using this variable using polarised quasi-elastic scattering with incident protons, giving a comparison for the current work. $\Omega$ can be calculated for NN events by the subtraction of the scattered nucleon energy $T_{N}^{\prime}$, from the incident neutron energy $T_{n}$. For NP events things are not so straightforward as the proton will lose energy in the scintillator wall before its energy is evaluated through time of flight between the first and second walls. Thus in addition to the scattered 

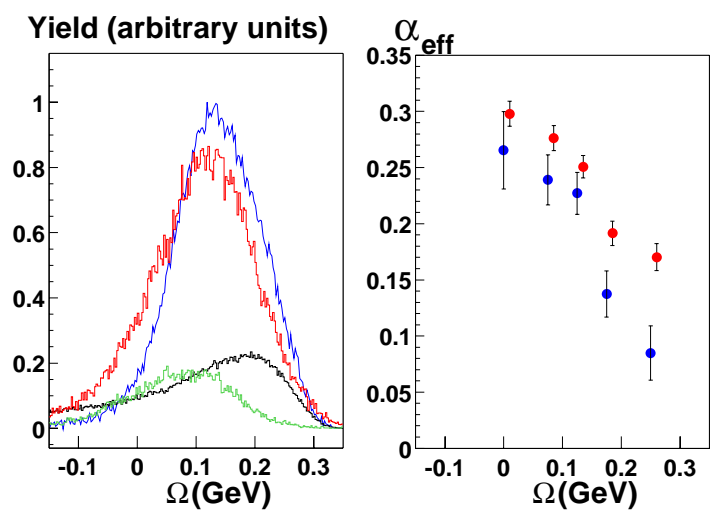

Figure 6.11: NN results for energy loss $\Omega$. The left hand plot shows the distribution for the all simulated events(red line), simulated $p\left(n, n^{\prime}\right) p^{\prime}$ events (green), real events(blue) and the random background(black line). The right hand plot shows the effective analysing power from the simulation(red points) and data(blue points)

proton energy, the energy deposited in the first wall $E_{d e p}^{1}$, should also be subtracted. At high energy losses final state interaction(FSI) or meson exchange current(MEC) effects may be significant and in this case the analysing power can be quite different to that from free nucleon-nucleon scattering.

\section{Yields as a function of $\Omega$}

The simulated distribution, which has mainly quasi-elastic events, has been shifted to larger energy loss by $30 \mathrm{MeV}$ for NN events and $25 \mathrm{MeV}$ for NP events in order to match approximately the peak positions. It has been observed in previous investigations of quasi-elastic nucleon scattering [78], that the peak in the $\Omega$ distribution is shifted from the position of free $(p, n)^{1}$ scattering which is a just dependent on the scattering angle, by around $25 \mathrm{MeV}$ for a ${ }^{12} \mathrm{C}$ target. The peak position for free and quasi-elastic scattering are the same in the simulation. Rosenfelder [79] showed that this is expected where distortion and correlation effects are neglected as is the case of the present model. Pandharipande et al [80] and Wambach [81] have suggested that the cause of the shift is the additional energy required to convert a proton to neutron in the presence of a nuclear potential. This however does not explain the shift in the $(n, n)$ channel, which has not previously been investigated, and no shift is observed in $(p, p)$ experiments [78].

\footnotetext{
${ }^{1}$ notation implies proton incident on the nucleus, neutron scattered out.
} 

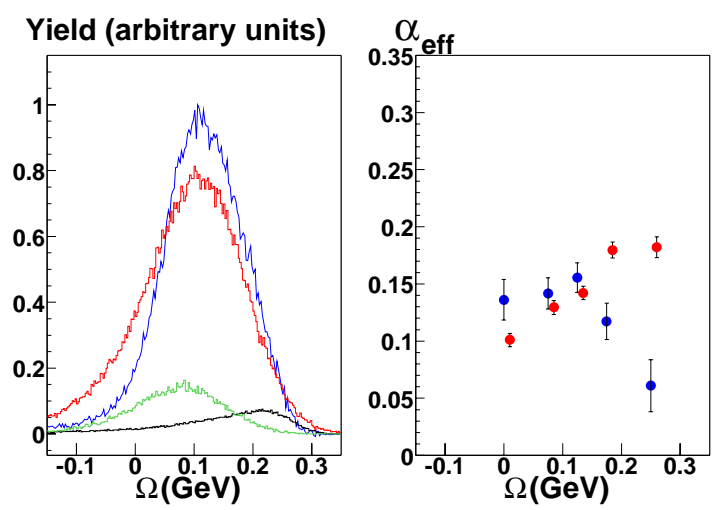

Figure 6.12: NP results for energy loss $\Omega$.The left hand plot shows the distribution for the all simulated events(red line), simulated $p\left(n, p^{\prime}\right) n^{\prime}$ events (green), real events(blue) and the random background(black line). The right hand plot shows the effective analysing power from the simulation(red points) and data(blue points).

\section{$\alpha_{e f f}$ as a function of $\Omega$}

The shape of the $\Omega$ dependence of the analysing power for NN events is well reproduced by the simulation, although it is systematically underestimated in all bins, in particular for the two highest energy loss points. For NP events, the simulation and data again agree well for $\Omega<0.13 \mathrm{GeV}$, but for $\Omega>0.13 \mathrm{GeV}$ the data and simulation diverge. The calculated increase in $\alpha_{\text {eff }}$ with $\Omega$ is related to the $\theta_{n}^{\prime}$ acceptance, as the energy loss of a projectile increases with increasing scattering angle. At high $\Omega$, quasi-elastic scattering is not a good approximation as additional energy may have been lost through FSI or MEC and in these cases one would expect a dilution of the analysing power, which is observed in the data. De Pace and Viviani [82] deduced that the effect of two-step processes (FSI) is to first order twice as significant for charge exchange processes, since these may proceed in either the first or second scattering.

The ${ }^{12} C(\vec{p}, n)$ reaction has been measured $[83,78]$ at similar nucleon energies to the present experiment and some results from [78] are given in figure 6.13 , showing differential cross sections and analysing power $\left(A_{y}\right)$ as a function of $\omega(\equiv \Omega)$. The displayed data was evaluated for two particular momentum transfers, (or equivalently scattering angles) and the cross-section plots compare the data with results of a model calculation for single (dashed curve) and twostep (dotted curve) scattering as well as their sum (full curve). The calculations have been shifted to match the broad measured quasi-elastic peak, as explained 

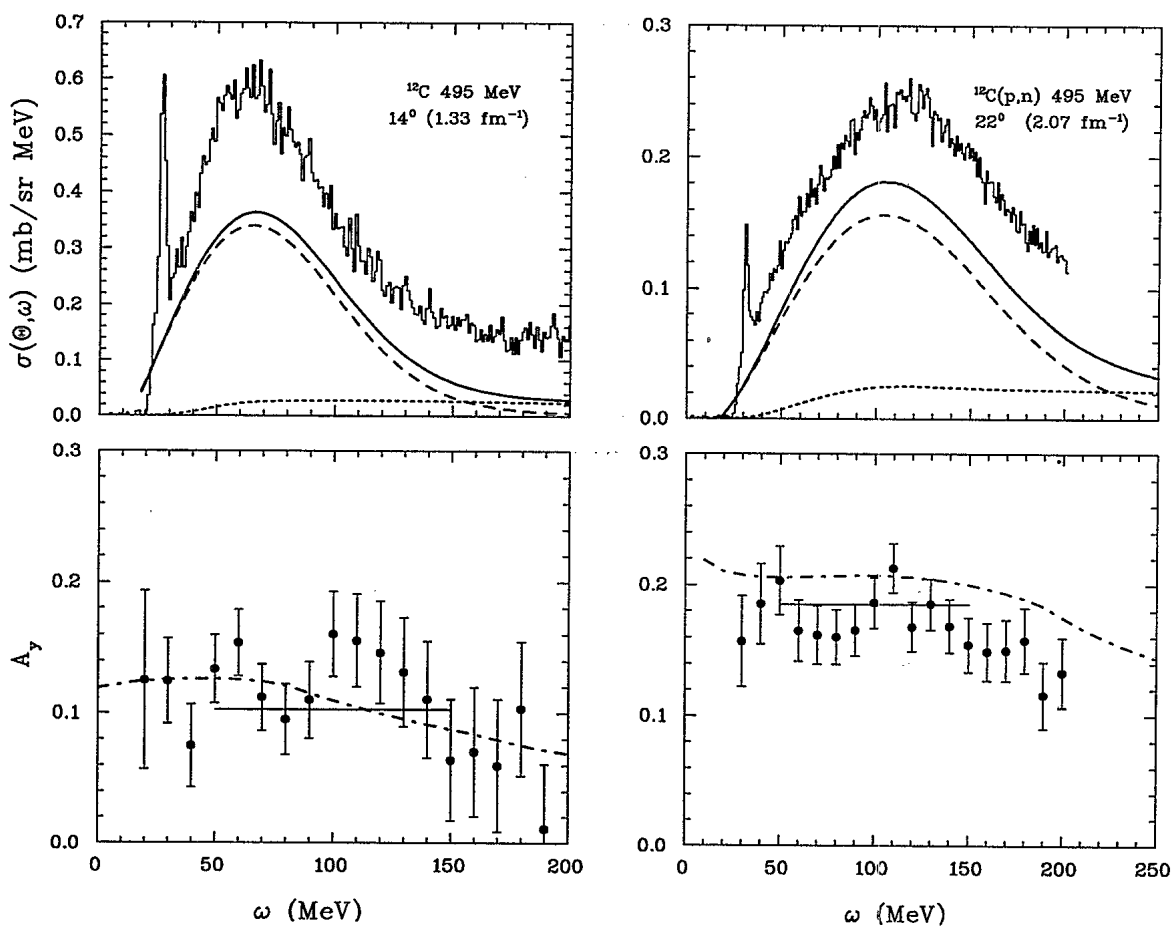

Figure 6.13: Differential cross sections and analysing powers for ${ }^{12} C(\vec{p}, n)$ from [78].

above. At high $\Omega$ two-step processes become important and the model significantly underestimates the measured cross-section in this region, implying either that two-step processes are underestimated or that other neglected nuclear effects are significant.

The $A_{y}$ plots (fig. 6.13), show the experimental results, along with a full line showing the result for free NN scattering and a dash-dotted line showing the calculations of [84]. In the region where two-step processes are predicted to dominate, the measured analysing power falls below the value of free NN scattering. In figure 6.12, the divergence of the measured data from simulated $\alpha_{\text {eff }}(\Omega)$, for $\Omega>0.13 \mathrm{GeV}$, would seem to be linked to the present model's neglect of final state interactions, or other nuclear effects such as meson exchange.

\subsubsection{Conclusions on the simulation}

The Monte Carlo simulation and experiment largely agree on kinematic distributions and effective analysing powers, particularly when detecting protons in the 
second wall of the polarimeter. This gives confidence that the analysing reactions are understood :

- Quasi-elastic scattering on carbon is the dominant analysing reaction in the polarimeter.

- Detecting protons in the second wall allows analysis of scattered neutrons that undergo charge exchange interactions in the first wall.

- The effective analysing power can be reasonably well modelled by assuming the free nucleon scattering analysing power and introducing Fermi smearing of the scattering angle.

- The NP data gives results more consistent with the simulation than the NN data. It therefore seems to be a "cleaner" polarised nucleon scattering signal.

- The simulation finds the analysing power for NP to be approximately $70 \%$ of that for NN, similar to the results of the data.

However these investigations have not yielded any clear kinematic cuts which might be used to enhance the neutron scattering asymmetry and as a result no kinematic cuts will be applied to the final analysis.

The models employed may be of use for planning future neutron polarimetry experiments in this or higher energy ranges. However for projectile energy loss $(\Omega)$, the simulation does not predict the measured peak position or high $\Omega, \alpha_{\text {eff }}$ behavior, which is consistent with the model's neglect of nuclear distortions and quasi-elastic multiple scattering(FSI). It would be possible to account for these effects in the quasi-elastic model by input of the measured $\Omega$ shift as a function of incident energy and scattering angle, and also by input of the FSI cross section which could be taken from calculations (e.g. [78]).

\subsection{Final Result}

The ratio of recoil neutron polarisation components $\frac{P_{x}^{n}}{P_{z}^{n}}$ has been determined in the reference frame defined by the recoil neutron (eqn. 5.9). To determine $G_{E}^{n}$ via equation 3.4 requires firstly the application of the nuclear structure corrections of section 5.6 and then the determination of two further factors: the neutron magnetic form factor $G_{M}^{n}$ and a kinematic factor $K\left(Q^{2}\right) . G_{E}^{n}$ is then given by: 

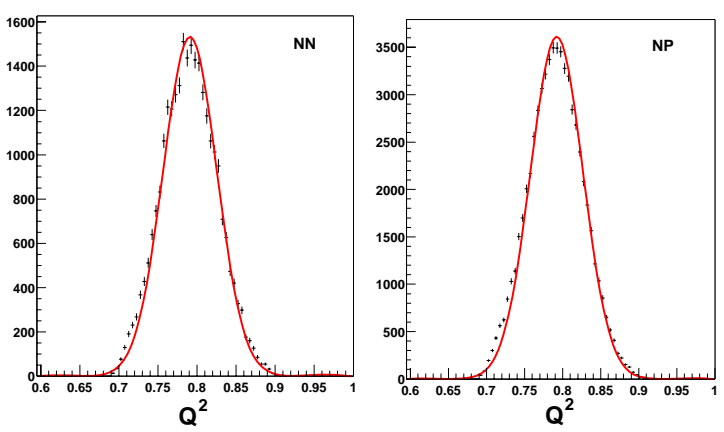

Figure 6.14: $Q^{2}$ distributions for sampled NN and NP distributions with Gaussian fits.

$$
G_{E}^{n}\left(Q^{2}\right)=-K\left(Q^{2}\right) G_{M}^{n}\left(Q^{2}\right)\left(\frac{P_{x}^{q}}{P_{z}^{q}}\right)^{B o r n}
$$

The distributions of $Q^{2}$ for the events sampled is shown in figure 6.14. Fitting with a Gaussian yields mean values $Q^{2}=0.79 \pm 0.03\left(\frac{\mathrm{GeV}}{\mathrm{c}}\right)^{2}$ for NN and $Q^{2}=$ $0.79 \pm 0.03\left(\frac{\mathrm{GeV}}{\mathrm{c}}\right)^{2}$ for NP with $\sigma$ the width of the Gaussian.

\subsection{1 $G_{M}^{n}\left(Q^{2}=0.79\left(\frac{G e V}{c}\right)^{2}\right)$}

The value for $G_{M}^{n}\left(Q^{2}=0.79\left(\frac{G e V}{c}\right)^{2}\right)$ used here has been taken from the continued fraction parameterisation of Kubon et al [14], which has been discussed in section 3.2. The parameterisation has the form:

$$
G_{M}^{n}\left(Q^{2}\right)=\frac{\mu_{n}}{1+\frac{Q^{2} b_{1}}{1+\frac{Q^{2} b_{2}}{1+\cdots}}}
$$

with the fitted parameters, $b_{1} \ldots b_{5}=3.26,-0.272,0.0123,-2.52,2.55\left(\frac{\mathrm{GeV}}{\mathrm{c}}\right)^{-2}$. The quoted error on the fitted function is $1.1 \%$, which gives $G_{M}^{n}(0.79)=-0.449 \pm$ 0.004 .

In previous double polarisation experiments the dipole form factor (eqn. 3.5) has been used to estimate $G_{M}^{n}$, which at this $Q^{2}$ gives a value of $\mu_{n} G^{D}(0.79)=$ -0.428 . The value from the constant fraction parameterisation is $4.9 \%$ larger. 

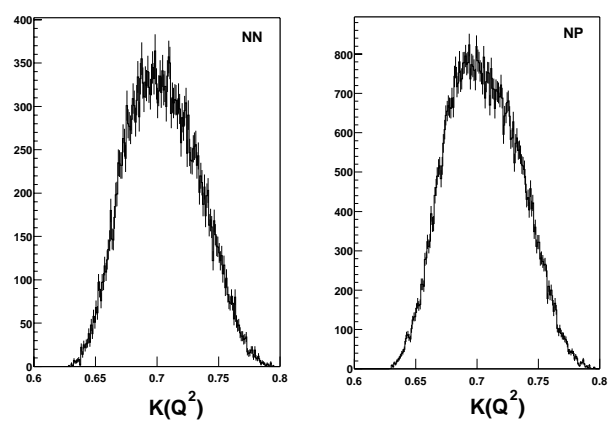

Figure 6.15: $K\left(Q^{2}\right)$ distributions for sampled NN and NP distributions.

\subsection{2 $K\left(Q^{2}\right)$}

The distribution of $K\left(Q^{2}\right)=\sqrt{\tau+\tau(1+\tau) \tan ^{2}\left(\frac{\theta_{e}}{2}\right)}$ over the complete data set is shown in figure 6.15. For determining $G_{E}^{n}$, the mean values of $K$ have been used $: K(0.79)=0.705 \pm 0.014$ for NN and $K(0.79)=0.705 \pm 0.012$ for NP.

\subsection{3 $G_{E}^{n}$}

A summary of the contributing factors and the results for $G_{E}^{n}$ is given in table 6.3, for NN and NP separately. The results for each are in good agreement with each other (within $1 \sigma$ ). Combining both these results leads to a final value of $G_{E}^{n}=(0.047 \pm 0.009)$ at $Q^{2}=(0.791 \pm 0.034)\left(\frac{\mathrm{GeV}}{\mathrm{c}}\right)^{2}$.

\subsubsection{Systematic Uncertainties}

One advantage of the present experimental technique is that a large number of systematic uncertainties cancel to first order in the polarisation ratio when equation 4.7 is used to calculate the asymmetry. The flipping of the beam helicity essentially gives two asymmetry experiments with the top and bottom second wall scintillators. Individually these experiments will have the same neutron detection efficiency for the two different helicity states and so the detection efficiency cancels in the asymmetry. Luminosity fluctuations are also negligible as the helicity is flipped on average every second.

An advantage the current A1 Hall MAMI experiments had over the previous MAMI A3 measurements was an accurate reconstruction of the reaction $Q^{2}$. The high resolution magnetic spectrometer gives a momentum resolution of below $10^{-4}$. 


\begin{tabular}{|c|c|c|}
\hline & $\mathrm{NN}$ & $\mathrm{NP}$ \\
\hline \hline$Q^{2}$ & $0.791 \pm 0.034$ & $0.792 \pm 0.034$ \\
\hline$\frac{P_{x}^{n}}{P_{z}^{n}}$ & $0.166 \pm 0.046$ & $0.128 \pm 0.037$ \\
\hline$f$ & $-0.0020 \pm 0.0001$ & $-0.0020 \pm 0.0001$ \\
\hline$\left(\frac{P_{x}^{q}}{P_{z}^{q}}\right)^{\text {full }}$ & $0.164 \pm 0.046$ & $0.126 \pm 0.037$ \\
\hline$\Delta R_{p}$ & $-0.0080 \pm 0.0003$ & $-0.0078 \pm 0.0003$ \\
\hline$\left(\frac{P_{x}^{q}}{P_{z}^{q}}\right)^{\text {Born }}$ & $0.172 \pm 0.046$ & $0.134 \pm 0.037$ \\
\hline$K\left(Q^{2}\right)$ & $0.705 \pm 0.014$ & $0.705 \pm 0.012$ \\
\hline$G_{M}^{n}\left(Q^{2}\right)$ & $-0.449 \pm 0.004$ & $-0.449 \pm 0.004$ \\
\hline$G_{E}^{n}\left(Q^{2}\right)$ & $0.054 \pm 0.014$ & $0.042 \pm 0.012$ \\
\hline
\end{tabular}

Table 6.3: Summary of contributing factors and final results. $\frac{P_{x}^{n}}{P_{z}^{n}}$ is taken from table 6.2; $\bar{f}$ (sec. 5.6.1) is added to this to give $\left(\frac{P_{x}^{q}}{P_{z}^{q}}\right)^{\text {full }} ; \Delta R_{p}$ (sec. 5.6.2) is added to this to give $\left(\frac{P_{q}^{q}}{P_{z}^{q}}\right)^{\text {Born }}$; eqn. 6.2 is then used to determine $G_{E}^{n}$.

Uncertainties can also arise from particle misidentification which can lead to measuring a combination of neutron and proton polarisation. The contribution of the misidentified protons was discussed in section 6.2 and found to be at most $1 \%$ for NP events and $0.5 \%$ for NN events. The mean polarisation ratio of protons is related to the ratio of form factors in the same way as for neutrons and using the result of [85] $\frac{\mu_{p} G_{E}^{p}}{G_{M}^{P}}=0.95 \pm 0.015$, gives $\left(\frac{P_{x}}{P_{z}}\right)^{p}=0.753$. Assuming this gives a $0.5 \%$ contribution to the NN events and a $1 \%$ contribution to the NP events, the measured $G_{E}^{n}$ is shifted by only -0.001 and -0.002 respectively, (i.e. $-2 \%$ and $-5 \%)$.

A similar uncertainty can occur from protons ejected from the liquid deuterium target undergoing a charge exchange interaction before reaching the first wall of the polarimeter, resulting in neutrons with essentially the same polarisation as the initial protons. The main source of such neutrons would be the lead shielding positioned in the gap at the entrance to the precession magnet. In the analysis of the $Q^{2}=0.3$ and $0.6\left(\frac{\mathrm{GeV}}{\mathrm{c}}\right)^{2}$ data [52] the combined yield of these neutrons and misidentified protons was estimated by analysing data taken with a liquid ${ }^{1} H$ target. The fraction of these false events was found to be $0.5 \%$ and $1.1 \%$ for NN and NP respectively, which is similar to the upper limit found for misidentified protons alone in this analysis. In [20] the systematic uncertainty from $p \rightarrow n$ reactions in the lead shielding was given as $1 \%$. It is hoped to eventually simulate this effect using the differential quasi-elastic $P b(p, n)$ cross 
section which has been measured [78] at similar incident energy. This would also require the simulation of the neutron double coincidence detection efficiency to determine the total yield of neutrons and in addition the ratio of quasi elastic $\left(e, e^{\prime} n\right)$ to $\left(e, e^{\prime} p\right)$ cross sections which have been determined in a measurement of $G_{M}^{n}$ analysis [14], to give the luminosity of protons incident from the target.

The experimental uncertainty in $G_{M}^{n}$ also leads to a systematic uncertainty in $G_{E}^{n}$. The fit by Kubon et al [14] on recent high precision measurements of $G_{M}^{n}$ is estimated to have an uncertainty of $1.1 \%$.

The systematic uncertainty on the precession angle $\chi$ is discussed in section 5.4.3. It is mainly due to the uncertainty of the integrated field which is $2 \%$.

Combining these contributions the overall systematic error is estimated to be ${ }_{-2.3 \%}^{+3.4 \%}$ for NN and ${ }_{-2.3 \%}^{+5.7 \%}$ for NP data and ${ }_{2.3 \%}^{4.6 \%}$ combined.

\section{5 $G_{E}^{n}$ versus $Q^{2}$}

\subsubsection{Experimental Data and Phenomenological Fits}

The result of this analysis gives the highest $Q^{2}$ data point obtained for a double polarisation experiment at MAMI. Figure 6.16 shows the current status of experimental data. The present $Q^{2}=0.8\left(\frac{\mathrm{GeV}}{\mathrm{c}}\right)^{2}$ data point is consistent with the trend of the previous data. After rising steeply to a maximum at $\sim 0.25\left(\frac{\mathrm{GeV}}{\mathrm{C}}\right)^{2}$, $G_{E}^{n}$ falls off slowly with $Q^{2}$ in the range $Q^{2}<1\left(\frac{\mathrm{GeV}}{\mathrm{c}}\right)^{2}$.

The displayed data set shown has been fitted with the commonly used parameterisation [21]:

$$
G_{E}^{n}\left(Q^{2}\right)=\frac{a \tau}{(1+b \tau)} G_{D}\left(Q^{2}\right) \text { with } G_{D}\left(Q^{2}\right)=\frac{1}{\left(1+\frac{Q^{2}}{0.71}\right)^{2}} \text { and } \tau=\frac{Q^{2}}{4 M_{n}^{2}}
$$

The derivative of 6.3 at $Q^{2}=0$ is related to the mean squared charge radius $\left\langle r_{E}^{2}\right\rangle_{n}$ as given in equation 2.1.5, so that :

$$
\left(\frac{d G_{E}^{n}(0)}{d Q^{2}}\right)=\frac{a}{4 M_{n}^{2}}=-\frac{1}{6}\left\langle r_{E}^{2}\right\rangle_{n}
$$

Using the precise measurement of [15]; $\left\langle r_{E}^{2}\right\rangle_{n}=-(0.113 \pm 0.003 \pm 0.004) \mathrm{fm}^{2}$, fixes $a=1.73\left(\frac{\mathrm{GeV}}{\mathrm{c}}\right)^{-2}$ which leads to a fitted value of $b=4.4 \pm 0.9\left(\frac{\mathrm{GeV}}{\mathrm{c}}\right)^{-2}$. 


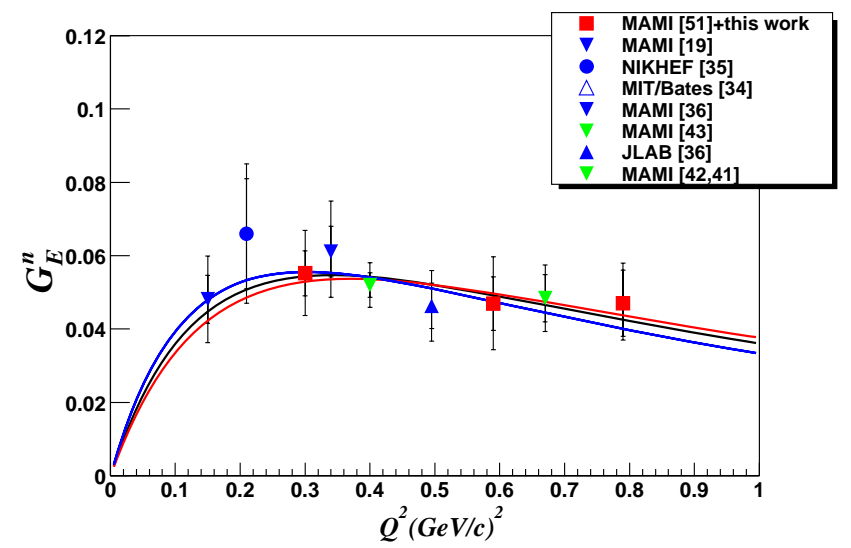

Figure 6.16: $G_{E}^{n}$ data from polarised experiments (see fig. 3.4 for references) including the new MAMI A1 data (red squares). The $Q^{2}=0.3$ and $0.6\left(\frac{\mathrm{GeV}}{\mathrm{c}}\right)^{2}$ data are from [52]. Also shown are the original ad hoc Galster fit [31] to unpolarised data (black line) and the new fit to the polarised data with one(red) and two(blue) free parameters.

On the other hand taking $a$ and $b$ as a free parameters results in the values $a=1.2 \pm 0.2\left(\frac{\mathrm{GeV}}{\mathrm{c}}\right)^{-2}$ and $b=7.9 \pm 3.3\left(\frac{\mathrm{GeV}}{\mathrm{c}}\right)^{-2}$. Which is not consistent with the measured $\left\langle r_{E}^{2}\right\rangle_{n}$, but as yet gives very little restriction to $\left\langle r_{E}^{2}\right\rangle_{n}$ as the data are at too high a $Q^{2}$.

These fits agree very well with the preferred Galster fit (sec. 3) where not allowing for a variation in the slope of $G_{E}^{n}$ at $Q^{2}=0$, essentially fixed $a=$ $-\frac{\mu_{n}}{\mu_{N}}=1.91\left(\frac{\mathrm{GeV}}{\mathrm{c}}\right)^{-2}$ and found $b=5.6\left(\frac{\mathrm{GeV}}{\mathrm{c}}\right)^{-2}$. This must be regarded as a coincidence though, the results of Galster cannot be taken too seriously due to the limitations of the deuteron wave functions available at the time.

\section{Pion Cloud and Constituent Quarks}

A recent paper by Friedrich and Walcher, which included the preliminary results of this analysis, has suggested a new parameterisation for $G_{E}^{n}$, which has a straightforward interpretation with respect to nucleon structure in terms of a pion cloud and constituent quarks [86]. They hypothesise that the Galster parameterisation does not describe the data ideally, in particular it does not allow for a perceived "bump" in the data around $0.2<Q^{2}<0.4\left(\frac{\mathrm{GeV}}{\mathrm{c}}\right)^{2}$. They also point out that there is no real physics motivation behind the Galster parameterisation as it merely allows the dipole parameterisation to tend to zero at $Q^{2}=0$. To fit 


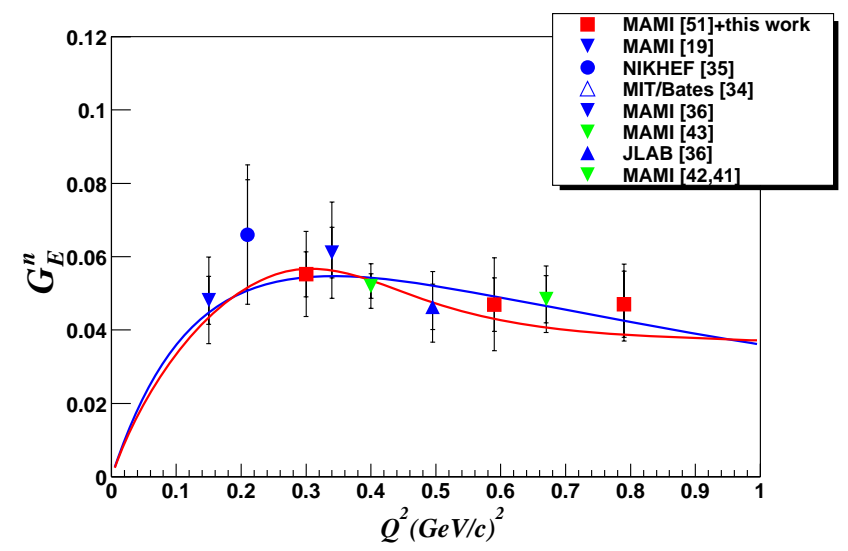

Figure 6.17: The double polarisation data with the Galster fit $(a=1.73, b=4.4)$ (blue) and the Friedrich-Walcher fit(red).

the "bump" they include an extra term to equation 6.3 :

$$
G_{E}^{n}\left(Q^{2}\right)=\frac{a Q^{2}}{\left(1+b Q^{2}+c Q^{10}\right)}+\frac{d Q^{2}}{\left(1+e Q^{2}\right)\left(1+f Q^{2}\right)^{2}}
$$

For their fit they fixed $a$ and $d$ to give the correct $\left\langle r_{E}^{2}\right\rangle_{n}, e$ and $f$ were fixed at $0.5\left(\frac{\mathrm{GeV}}{\mathrm{c}}\right)^{-2}$, the remaining parameters $\mathrm{b}$ and $\mathrm{c}$ were found to be $6.02\left(\frac{\mathrm{GeV}}{\mathrm{c}}\right)^{-2}$ and $229\left(\frac{\mathrm{GeV}}{\mathrm{c}}\right)^{-10}$ respectively. Both the Galster fit of section 6.5.1 and the Friedrich-Walcher fit are compared to the data in figure 6.17. The experimental uncertainties are such that the data shows no clear preference for either curve.

After establishing that such a fit gives a reasonable agreement with the data it was then shown that its features can be described by viewing the nucleon as a superposition of a bare nucleon with a pion cloud:

$$
n=a_{n} n^{0}+b_{n}\left(p^{0}+\pi^{-}\right) \text {and } p=a_{p} p^{0}+b_{p}\left(n^{0}+\pi^{+}\right)
$$

The form factors are then given by the sum of the form factors of the constituents, which for the bare nucleons are related to dipole form factors of the constituent quarks. The "bump" in the $G_{E}^{n}$ data is then a result of the $\left(p^{0}+\pi^{-}\right)$contribution, while the large $Q^{2}>1$ behaviour is dominated by the bare neutron.

An advantage of this picture is that it fits all four nucleon form factors $G_{E}^{p}$, $G_{M}^{p}, G_{E}^{n}$ and $G_{M}^{n}$ remarkably well. $G_{E}^{p}, G_{M}^{p}$ and $G_{M}^{n}$ have been measured to a much greater accuracy than $G_{E}^{n}$ and these all show a similar structure in the 

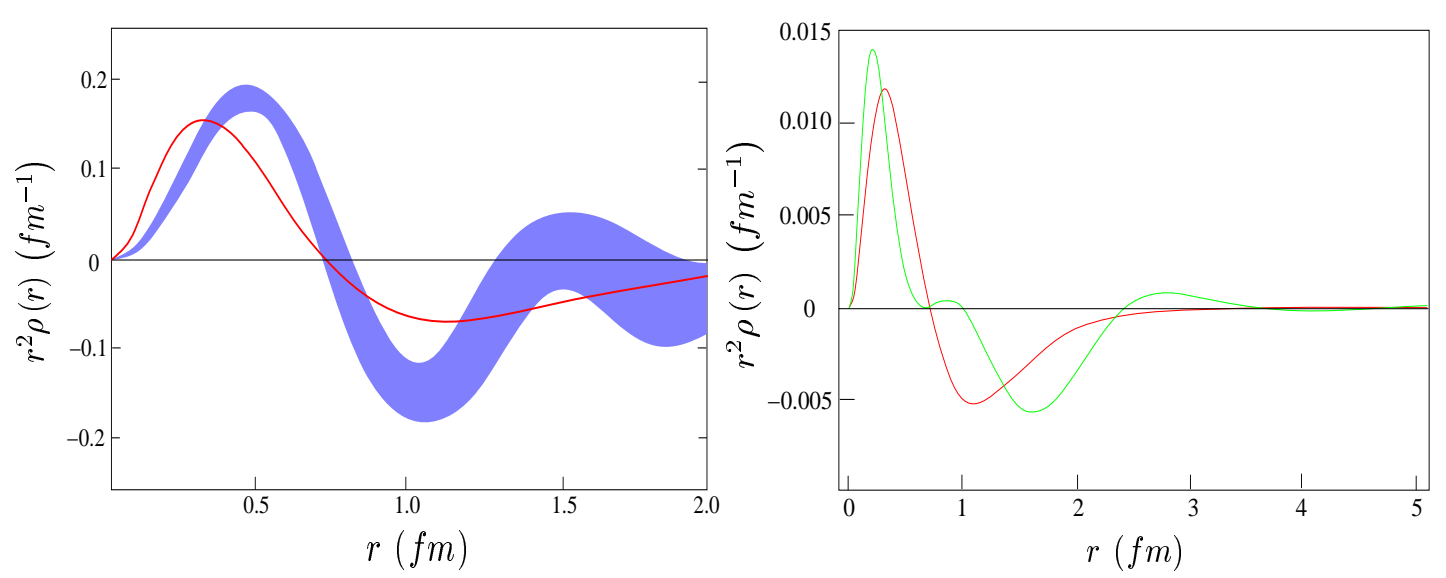

Figure 6.18: The neutron charge distribution taken from Kelly [18] (left) and Friedrich-Walcher [86] (right). (The difference in y-scale is possibly due to a factor of $4 \pi$ ). The distribution from the Galster fit to double polarisation data is shown in red in both cases.

$Q^{2} \sim 0.3\left(\frac{\mathrm{GeV}}{\mathrm{c}}\right)^{2}$ region.

\section{Neutron Charge Distribution}

One of the key reasons for interest in $G_{E}^{n}\left(Q^{2}\right)$ is it's relationship to the distribution of charge in the neutron $\rho(r)$ (sec. 2.1.6). Kelly [18] asserts that this relationship is not given by a simple Fourier transform, but in fact a relativistic Fourier transform is required. The result of such a transform is shown in figure 6.18(left) along with the non-relativistic Fourier transform of a Galster fit. The filled region indicates the uncertainty due to experimental accuracy and transformation procedure.

Friedrich and Walcher also transform their $G_{E}^{n}\left(Q^{2}\right)$ results to $\rho(r)$ using nonrelativistic Fourier transform as shown in figure 6.18(right), for their fit given in the previous section. With respect to their model a small $r$ peak is given mainly by the bare neutron and has a form similar to that of the Galster line, while the extended negative charge distribution is a result of contributions from the negative pion cloud and differs significantly from the Galster curve.

Overall the interpretation of the neutron charge distribution and indeed how it relates exactly to the electric form factor is still unclear and although much progress is being made in this area more accurate data is still essential. 


\subsubsection{Theoretical Models}

Quantum Chromodynamics(QCD) is a quantum field theory where quarks, the elementary constituents of hadrons, interact via the exchange of spin-one bosons called gluons. The quarks are subject to two main constraints: that they are confined in colourless hadronic states ${ }^{2}$; that they interact weakly at very small distances(asymptotic freedom). At very high momentum transfer equivalent to very small distances, QCD may be solved pertubatively. The scaling behaviour of the form factors in the limit $Q^{2} \rightarrow \infty$ can be predicted from pertubative QCD (pQCD). Brodsky and Lepage [87] predicted a $Q^{-4}$ and $Q^{-6}$ dependence for $F_{1}$ and $F_{2}$ respectively (see eqn. 2.15) and therefore a $Q^{2}$ dependence in the ratio : $\frac{Q^{2} F_{2}}{F_{1}}=$ const. More recently Ralston [88], from consideration of non Swave components of quark orbital angular momentum, has given a modified $Q^{2}$ dependence of the ratio showing $\frac{Q F_{2}}{F_{1}}=$ const. This is consistent with recent high $Q^{2}$ measurements of the proton form factor ratio [89].

At the nucleonic scale $(\sim 1 \mathrm{fm})$ QCD may not be solved pertubatively and one has to resort to models to describe the nucleon. Any credible model must be able to predict the nucleon form factors. In general nucleon models are particularly sensitive to the small neutron electric form factor and a large variety of models and predictions for $G_{E}^{n}$ can be found in the literature; all the models are effective theories that have many parameters which are adjusted to fit the data. A selection is shown in figure 6.19, along with the model-independent double-polarisation experimental data.

The best comparison to the fit at both high and low $Q^{2}$ is given by the Soliton model of Holzwarth [1] (e in fig. 6.19) which also gives excellent agreement with the proton form factor ratio [89]. Holzwarth modifies the standard Soliton model which is an effective (pionic) field theoretical model, by inclusion of vector meson dominance contributions (VMD). VMD allows the virtual photon to couple to the nucleon through vector mesons (e.g $\rho, \omega, \phi)$, which have the same spin-parity as the photon.

The dispersion theory result (f) [90] and the Goldstone-Boson Exchange Constituent Quark Model (GBECQM) (c) [91] both yield similar results. Though the shape is similar to the Soliton model the curves are systematically below the data. The Dispersion Theory approach is an attempt to simultaneously parameterise all four of the nucleon form factors by a fit of the available data subject

\footnotetext{
${ }^{2}$ Quarks carry a colour charge of either red, blue or green.
} 
to the constraints: inclusion of the two-pion continuum in the isovector spectral functions, the neutron charge radius determined from low energy neutron atom scattering and the pQCD scaling. It is not surprising then that this parameterisation underestimates $G_{E}^{n}$, as the parameterisation of Platchkov et al with the Paris potential [21] (fig. 3.4) which is well below the double polarisation data was used for the fitting.

In the GBECQM the effective degrees of freedom are constituent quarks ${ }^{3}$ and Goldstone-boson fields, the nucleons can then be considered as systems of three constituent quarks interacting through the exchange of Goldstone-bosons, which manifest themselves as an octet of pseudoscalar mesons.

The rest of the models fail to predict the clear maximum in the measured $G_{E}^{n}$ at $Q^{2} \simeq 0.3\left(\frac{\mathrm{GeV}}{\mathrm{c}}\right)^{2}$, instead they predict $G_{E}^{n}$ increases in the range $0<Q^{2}<$ $1\left(\frac{\mathrm{GeV}}{\mathrm{c}}\right)^{2}$. For $Q^{2}>0.3\left(\frac{\mathrm{GeV}}{\mathrm{c}}\right)^{2}$ the model of Cardarelli and Simula [92](b) which is a relativistic constituent quark model fits the data reasonably well. In addition to constituent quarks this model allows the breaking of SU(6) symmetry, thus allowing a non-zero $G_{E}^{n}$.

The VMD model of Gari and Krumpelman [93](d), which includes coupling to the $\phi$ meson and also assumes the pQCD scaling of Brodsky and Lepage, is low for $Q^{2}<0.3\left(\frac{\mathrm{GeV}}{\mathrm{c}}\right)^{2}$ and high for $Q^{2}>0.8\left(\frac{\mathrm{GeV}}{\mathrm{c}}\right)^{2}$. However, it clearly fits the data better than model of the same authors with no $\phi$ meson [94] (a).

To summarise only the Soliton model, dispersion theory and the CQGBE parameterisations give a reasonable shape for $G_{E}^{n}$ in the range $0<Q^{2}<1\left(\frac{\mathrm{GeV}}{\mathrm{c}}\right)^{2}$. The Soliton model also agrees well with the magnitude of the experimental data.

\footnotetext{
${ }^{3}$ Constituent quarks are assumed to be pointlike particles with masses $\sim 300 \mathrm{MeV}$.
} 


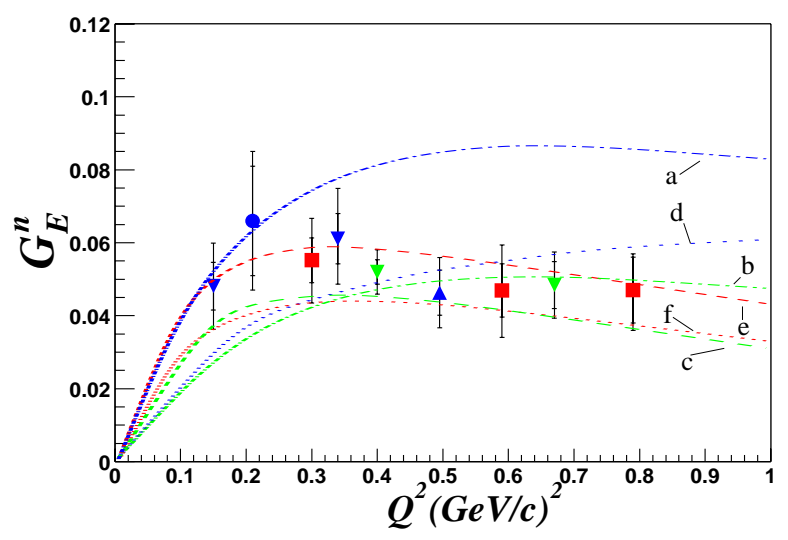

Figure 6.19: Comparison of various theoretical models of the nucleon with the model-independent double polarisation data. (a)Gari-Krumpelman(Vector Meson Dominance no $\Phi$ meson) [94]; (b)Cardarelli-Simula(with Constituent Quarks) [92]; (c)Goldstone-Boson Exchange Constituent Quark Model [91]; (d)GariKrumpelman(VMD with $\phi$ meson) [93]; (e)Soliton [1]; (f)Mergell-MeissnerDrechsel(dispersion theory) [90]; .

\subsection{Conclusions}

The spin precession technique has been used to determine the polarisation ratio of the recoil neutron in the $D\left(\vec{e}, e^{\prime} \vec{n}\right) p$ reaction at $Q^{2}=0.79\left(\frac{\mathrm{GeV}}{\mathrm{c}}\right)^{2}$. From this ratio the neutron electric form factor $G_{E}^{n}$ has been extracted :

$$
G_{E}^{n}\left(0.79\left(\frac{\mathrm{GeV}}{\mathrm{c}}\right)^{2}\right)=0.047 \pm 0.009(\text { stat }) \pm_{0.001}^{0.002}(\text { sys })
$$

This result for $G_{E}^{n}$ includes full corrections for deuteron structure effects based on the model of Arenhövel [28]. In the course of the analysis a detailed investigation has been made into the functionality of the neutron polarimeter. The effective analysing powers measured compare well with those calculated from a Monte-Carlo simulation which assumes a quasi-elastic scatter of neutrons on Carbon as the main analysing reaction channel in the polarimeter. Nucleon-nucleon scattering events, with and without charge exchange in the neutron polarimeter, were analysed separately. The results of both reaction channels agree well.

This measurement had the advantage over the previous MAMI spin precession measurements (by the A3 collaboration) of utilising one of the A1 high resolution magnetic spectrometers giving an accurate $Q^{2}$ reconstruction and eliminating 
inelastic electron scattering events, thus reducing a large source of systematic uncertainty.

The best fit to the current model-independent data is given by the Soliton model of Holzwarth [1] which agrees well with both the shape and magnitude of the experimental data.

Future prospects for $G_{E}^{n}$ are very promising. In parallel with this analysis data points at $Q^{2}=0.30$ and $0.59\left(\frac{\mathrm{GeV}}{\mathrm{C}}\right)^{2}[52]$ were also analysed by Michael Seimetz for the Mainz A1 collaboration. At Jefferson Lab. Hall C analyses of $G_{E}^{n}$ data at $Q^{2}=0.4,1.14$ and $1.47\left(\frac{\mathrm{GeV}}{\mathrm{C}}\right)^{2}$, also using a spin precession technique are well underway. Proposed experiments [95] at Jefferson Lab. Hall A using the ${ }^{3} \overrightarrow{H e}(\vec{e}, n) p$ reaction have been accepted and will measure $G_{E}^{n}$ up to $Q^{2} \sim 3.5\left(\frac{\mathrm{GeV}}{\mathrm{c}}\right)^{2}$ allowing a first test of the pQCD scaling behavior of this observable at large $Q^{2}$. The Glasgow Nuclear Physics Group are major players in this experiment, continuing their involvement with $G_{E}^{n}$ measurements. 


\section{Appendix A}

\section{Asymmetry Errors}

Care has to be taken when propagating the statistical errors in an asymmetry calculation. The general case for an asymmetry defined as, $A=\frac{B-C}{B+C}$, has,

$$
\begin{aligned}
\left(\sigma^{A}\right)^{2} & =\left(\frac{\partial A}{\partial B}\right)^{2}\left(\sigma^{B}\right)^{2}+\left(\frac{\partial A}{\partial C}\right)^{2}\left(\sigma^{C}\right)^{2} \\
& =4\left[\frac{C^{2}\left(\sigma^{B}\right)^{2}+B^{2}\left(\sigma^{C}\right)_{C}^{2}}{(C+B)^{4}}\right]
\end{aligned}
$$

as $\left(\frac{\partial A}{\partial B}\right)=\frac{2 C}{(B+C)^{2}}$ and $\left(\frac{\partial A}{\partial C}\right)=\frac{-2 B}{(B+C)^{2}}$.

The asymmetry is calculated in this analysis from a fit to its distribution in $\phi_{n}^{\prime}$, therefore the asymmetry and error must be calculated for each bin of a $\phi_{n}^{\prime}$ histogram, this is denoted $A_{i}$ and $\sigma_{i}^{A}$ for bin $i$.

$B_{i}$ and $C_{i}$ are given by combinations of the $N\left(\phi_{n}^{\prime}\right)$ distribution histograms, from eqn. (5.10),

$$
\begin{aligned}
& B_{i}=\sqrt{B_{i}^{12}}=\sqrt{B_{i}^{1} B_{i}^{2}}=\sqrt{N_{i}^{+}\left(\phi_{n}^{\prime}\right) N_{i}^{-}\left(\phi_{n}^{\prime}+\pi\right)} \\
& C_{i}=\sqrt{C_{i}^{12}}=\sqrt{C_{i}^{1} C_{i}^{2}}=\sqrt{N_{i}^{+}\left(\phi_{n}^{\prime}+\pi\right) N_{i}^{-}\left(\phi_{n}^{\prime}\right)}
\end{aligned}
$$

where $i$ again indicates the histogram bin. First the errors $\sigma_{i}^{N}$, of the $N_{i}$ histograms were found from the prompt and random histograms, the errors for which are described in sec. A.1 : 


$$
\begin{gathered}
N_{i}=P P_{i}-P R_{i}^{\prime}-R P_{i}^{\prime}+R R_{i}^{\prime} \\
\left(\sigma_{i}^{N}\right)^{2}=\left(\sigma_{i}^{P P}\right)^{2}+\left(\sigma_{i}^{P R^{\prime}}\right)^{2}+\left(\sigma_{i}^{R P^{\prime}}\right)^{2}+\left(\sigma_{i}^{R R^{\prime}}\right)^{2}
\end{gathered}
$$

The error of the products $B_{i}^{12}$ and $C_{i}^{12}$ are then found from the correct combination of $N_{i}$ histograms. So if $B_{i}^{1}=N_{i}^{+}\left(\phi_{n}^{\prime}\right)$ etc. (eqns. A.3-A.4),

$$
\left(\sigma_{i}^{B 12}\right)^{2}=\left(\sigma_{i}^{B 1}\right)^{2}\left(B_{i}^{2}\right)^{2}+\left(\sigma_{i}^{B 2}\right)^{2}\left(B_{i}^{1}\right)^{2}
$$

The error of $B_{i}$, which is the square root of $B_{i}^{12}$, is then:

$$
\left(\sigma_{i}^{B}\right)^{2}=\frac{1}{2} \frac{\sigma_{i}^{B 12}}{\sqrt{B^{12}}}
$$

So if the errors for $C_{i}$ are calculated in the same way, the error for each asymmetry bin $\sigma_{i}^{A}$ are given by eqn. (A.2). The error on the overall asymmetry is given by the resulting sinusoidal fit.

\section{A.1 Error on Random Distributions}

There are two contributions to the error on the subtracted random $\phi_{n}^{\prime}$ histograms introduced in section 5.5.1, the first is from the shape, the second from the normalisation. The shape comes from the random $\phi_{n}^{\prime}$ histograms $R_{i}$ (any of $R P_{i}, P R_{i}$ or $R R_{i}$ ), which are normalised to the integral of the background fit $f(t)$ over the prompt timing regions $\left(T_{l} \rightarrow T_{u}\right)$ to give a random histogram $R_{i}^{\prime}$, for the prompt region :

$$
R_{i}^{\prime}=W R_{i} \text { where } W=\frac{\int_{T_{l}}^{T_{u}} f(t) d t}{\sum_{i} R_{i}} \frac{1}{b_{w}}=\frac{F}{R_{T}}
$$

where $b_{w}$ is the bin width of the histogram used to fit $f(t)$. If the background function is a 3rd degree polynomial :

$$
\begin{aligned}
f(t) & =p_{0}+p_{1} t+p_{2} t^{2}+p_{3} t^{3} \\
\Rightarrow F & =\int_{T_{l}}^{T_{u}} f(t) d t=\left[p_{0} t+p_{1} \frac{x^{2}}{2}+p_{2} \frac{x^{3}}{3}+p_{3} \frac{x^{4}}{4}\right]_{T_{l}}^{T_{u}} \\
& =\left(T_{u}-T_{l}\right) p_{0}+\frac{1}{2}\left(T_{u}^{2}-T_{l}^{2}\right) p_{1}+\frac{1}{3}\left(T_{u}^{3}-T_{l}^{3}\right)+\frac{1}{4}\left(T_{u}^{4}-T_{l}^{4}\right)
\end{aligned}
$$


The error in $F, \sigma_{F}$, is then calculated from the parameter errors $\sigma_{p}$ :

$$
\begin{aligned}
\sigma_{F}^{2} \cdot b_{w} & =\left(\frac{\partial F}{\partial p_{0}}\right)^{2} \sigma_{p_{0}}^{2}+\left(\frac{\partial F}{\partial p_{1}}\right)^{2} \sigma_{p_{1}}^{2}+\left(\frac{\partial F}{\partial p_{2}}\right)^{2} \sigma_{p_{2}}^{2}+\left(\frac{\partial F}{\partial p_{3}}\right)^{2} \sigma_{p_{3}}^{2} \\
& =\left(T_{u}-T_{l}\right)^{2} \sigma_{p_{0}}^{2}+\frac{1}{2}\left(T_{u}^{2}-T_{l}^{2}\right)^{2} \sigma_{p_{1}}^{2}+\frac{1}{3}\left(T_{u}^{3}-T_{l}^{3}\right)^{2} \sigma_{p_{2}}^{2}+\frac{1}{4}\left(T_{u}^{4}-T_{l}^{4}\right)^{2} \sigma_{p_{4}}^{2}
\end{aligned}
$$

The error in the integral of the $R_{i}$ histogram is taken as square root of the integral, $\sigma_{R_{T}}=\sqrt{R_{T}}$. The error in the weight is then :

$$
\begin{aligned}
\left(\sigma^{W}\right)^{2} & =\left(\frac{\partial W}{\partial F}\right)^{2} \sigma_{F}^{2}+\left(\frac{\partial W}{\partial R_{T}^{\prime}}\right)^{2} \sigma_{R_{T}}^{2} \\
& =\frac{1}{R_{T}^{2}} \sigma_{F}^{2}+\left(\frac{W}{R_{T}}\right)^{2} \sigma_{R_{T}}^{2}
\end{aligned}
$$

This is the error in $W$ integrated over all $\phi_{n}^{\prime}$ bins. However the error has to be applied to $R_{i}^{\prime}$ bin by bin to allow for a proper fit to $A_{i}$, therefore the error in $W$ has to be scaled for each bin in such a way as to give the correct integrated error. Assuming the fractional error on $W$ for each bin is proportional to the fractional error of the bin content $R_{i}, \sigma_{i}^{R}=\sqrt{R_{i}}$ and that $W$ is constant over all bins :

$$
\frac{\sigma_{i}^{W}}{W}=\frac{k}{\sqrt{R_{i}}}
$$

where $k$ is a normalisation constant. The sum of the square of the errors of all the bins must equal the square of the integrated error $\left(\sigma^{W}\right)^{2}$, so :

$$
\begin{gathered}
\left(\sigma^{W}\right)^{2}=\sum_{i} \frac{k^{2} W^{2}}{R_{i}} \\
\Rightarrow k^{2}=\frac{\left(\sigma^{W}\right)^{2}}{W^{2}}\left(\sum_{i} \frac{1}{R_{i}}\right)^{-1} \\
\Rightarrow\left(\sigma_{j}^{W}\right)^{2}=\left(\sum_{i} \frac{1}{R_{i}}\right)^{-1} \frac{\left(\sigma^{W}\right)^{2}}{R_{j}}
\end{gathered}
$$

Now the error on $R_{i}^{\prime}$ can be calculated :

$$
\begin{aligned}
\left(\sigma_{i}^{R^{\prime}}\right)^{2} & =\left(\frac{\partial R_{i}^{\prime}}{\partial R_{i}}\right)^{2}\left(\sigma_{i}^{R^{\prime}}\right)^{2}+\left(\frac{\partial R_{i}^{\prime}}{\partial W}\right)^{2}\left(\sigma_{i}^{W}\right)^{2} \\
& =W^{2}\left(\sigma_{i}^{R^{\prime}}\right)^{2}+R_{i}^{2}\left(\sigma_{i}^{W}\right)^{2}
\end{aligned}
$$


After this procedure the random histograms $R_{i}^{\prime}$ can be used for the random subtraction. 


\section{Appendix B}

\section{Reaction Kinematics}

\section{B.1 Electron Scattering}

For electron scattering in a Coulomb potential, with the initial and final electron four-vectors : $p^{i}=\left(E_{e}, \underline{k}\right) p^{f}=\left(E_{e}^{\prime}, \underline{k^{\prime}}\right)$

Conservation of Energy and Momentum :

$$
E_{e}=E_{e}^{\prime} \text { and }|\underline{k}|=\left|\underline{k}^{\prime}\right|
$$

Scalar product of initial and final momenta :

$$
\underline{k} \cdot \underline{k^{\prime}}=|\underline{k}|\left(1-2 \sin ^{2} \frac{\theta_{e}}{2}\right)
$$

Momentum transfer :

$$
|\underline{q}|=\left|\underline{k^{\prime}}-\underline{k}\right|=2|k| \sin \frac{\theta_{e}}{2}
$$

For electron scattering from a point-like spin- $\frac{1}{2}$ particle and the nucleon fourvectors $P^{i}=\left(E_{N}, \underline{P}\right)$ and $P^{f}=\left(E_{N}^{\prime}, \underline{P^{\prime}}\right)$, with $\approx$ implying in the relativistic limit $\frac{m_{e}}{E_{e}} \ll 1$.

The virtual photon four-momentum $q=(\omega, \underline{q})$ :

$$
q=p-p^{\prime}=P-P^{\prime}
$$

The squared four-momentum transfer :

$$
q^{2}=\omega^{2}-\underline{q}^{2} \equiv-Q^{2} \approx-4 E_{e} E_{e}^{\prime} \sin ^{2} \frac{\theta_{e}}{2}<0
$$




$$
q^{2}=\left(p^{f}-p^{i}\right)^{2}=p^{f 2}+p^{i 2}-2 p^{f} \cdot p^{i}=2\left(m_{e}^{2}-p^{f} \cdot p^{i}\right)
$$

Conservation of energy gives :

$$
\begin{gathered}
E_{e}^{\prime}=\frac{E_{e}}{1+\frac{2 E_{e}}{M_{N}} \sin \frac{\theta_{e}}{2}} \\
M_{N}\left(E_{e}-E_{e}^{\prime}\right)=E_{e} E_{e}^{\prime}-|\underline{k}|\left|\underline{k^{\prime}}\right| \cos \theta_{e}-m_{e}^{2} \\
\approx E_{e} E_{e}^{\prime}\left(1-\cos \theta_{e}\right) \\
=2 E_{e} E_{e} \sin ^{2} \frac{\theta_{e}}{2}
\end{gathered}
$$

\section{B.2 $D\left(e, e^{\prime} n\right) p$}

For quasielastic scattering there are 9 degrees of freedom in the final state, i.e. the three components of the momentum of the three particles in the final state $\underline{p_{e}^{\prime}}, \underline{p_{n}^{\prime}}$ and $p_{p}^{\prime}$. The electron spectrometer determines $\left.3 \underline{p_{e}}\right)$ and the polarimeter determines $3\left(\underline{p_{p}^{\prime}}\right)$; in addition the energy and momentum conservation restrict 4 , giving 10 , so we have overdetermined the kinematics. This allows us to calculate one of the 6 degrees we measure from the other 5 we measure, which is useful because we can thus get a more accurate deterimination of the neutron momentum $\left|\underline{p_{n}}{ }^{\prime}\right|$.

The 4-vectors of the initial (electron, deuteron) and final (scattered electron, recoil neutron and spectator proton) states are written as:

$$
e=\left(E_{e}, \underline{p_{e}}\right), d=\left(m_{d}, 0\right), e^{\prime}=\left(E_{e}^{\prime}, \underline{p_{e}^{\prime}}\right), n=\left(E_{n}^{\prime}, \underline{p_{n}^{\prime}}\right), p=\left(E_{p}^{\prime}, \underline{p_{p}^{\prime}}\right)
$$

Then energy and momentum conservation give,

$$
\begin{aligned}
e+d & =e^{\prime}+p+n \\
\Rightarrow p^{2} & =(q+d-n)^{2} \text { with } q=e-e^{\prime}=(\omega, \underline{q}) \\
\Rightarrow m_{p}^{2} & =q^{2}+d^{2}+n^{2}+2 d \cdot q+2 n \cdot q+2 d \cdot n \\
\Rightarrow m_{p}^{2} & =q^{2}+m_{d}^{2}+m_{n}^{2}+2 m_{d} \omega+-2 E_{n} m_{d}-2 E_{n} \omega+2 \underline{p_{n}} \cdot \underline{q} \\
\Rightarrow 2 E_{n} \gamma-2\left|\underline{p_{n}^{\prime}}\right| \alpha & =q^{2}+m_{d}^{2}+m_{n}^{2}-m_{p}^{2}+2 m_{d} \omega=\beta \\
\Rightarrow E_{n} & =\frac{\beta+2\left|\underline{p_{n}^{\prime}}\right| \alpha}{2 \gamma}
\end{aligned}
$$




$$
\begin{aligned}
\Rightarrow\left|\underline{p_{n}^{\prime}}\right|^{2}+m_{n}^{2} & =\frac{1}{(2 \gamma)^{2}}\left(\beta^{2}+4\left|\underline{p_{n}^{\prime}}\right|^{2} \alpha^{2}+4 \alpha \beta\left|\underline{p_{n}^{\prime}}\right|\right) \\
\Rightarrow 0 & =\left(1-\frac{\alpha^{2}}{\gamma^{2}}\right)\left|\underline{p_{n}^{\prime}}\right|^{2}-\left(\frac{\alpha \beta}{\gamma^{2}}\right)\left|\underline{p_{n}^{\prime}}\right|+\left(m_{n}^{2}-\frac{\beta^{2}}{4 \gamma^{2}}\right) \\
\Rightarrow\left|\underline{p_{n}^{\prime}}\right| & =\frac{\left(\frac{\alpha \beta}{\gamma^{2}}\right)+2 \sqrt{\left(m_{n}^{2}\left(\frac{\alpha^{2}}{\gamma^{2}}-1\right)+\frac{\beta^{2}}{4 \gamma^{2}}\right)}}{2\left(1-\frac{\alpha^{2}}{\gamma^{2}}\right)}
\end{aligned}
$$

Where $\alpha=\underline{\hat{p}_{n}} \cdot \underline{q}=q_{x} \sin \theta_{n} \cos \phi_{n}+q_{y} \sin \theta_{n} \sin \phi_{n}+q_{z} \cos \phi_{n}$ and $\gamma=m_{d}+\omega$. 


\section{Appendix C}

\section{Density Matrices, Structure Functions}

\section{C.1 Virtual Photon Density Matrix}

The non-zero longitudinal/transverse $\left(\rho_{L / T}\right)$ components of the virtual photon density matrix $\rho=\rho_{0}+h \rho^{\prime}$, with $h$ the beam helicity, are given in terms of the electron kinematics :

$$
\begin{array}{r}
\rho_{L}=q^{2} \frac{\xi^{2}}{2 \eta} \quad \rho_{L T}=q^{2} \frac{\xi}{\eta} \sqrt{\frac{(\xi+\eta)}{8}} \\
\rho_{T}=\frac{1}{2} q^{2}\left(1+\frac{\xi}{2 \eta}\right) \quad \rho_{T T}=-q^{2} \frac{\xi}{4 \eta} \\
\rho_{L T}^{\prime}=\frac{1}{2} q^{2} \frac{\xi}{\sqrt{2 \eta}} \quad \rho_{T}^{\prime}=\frac{1}{2} q^{2} \sqrt{\frac{(\xi+\eta)}{\eta}}
\end{array}
$$

with $\xi=\frac{q^{2}}{q^{2}}$ and $\eta=\tan ^{2}\left(\frac{\theta_{e}}{2}\right) ; q$ and $\underline{q}$ are the four and three momentum of the virtual photon respectively and $\theta_{e}$ is the electron scattering angle.

\section{C.2 Nucleon Structure Functions}

The structure functions $f$ are related to the nucleon current $J$, and density matrix $\rho_{N}$ :

$$
f_{L}=X_{00} \quad f_{T}=X_{x x}+X_{y y} \quad f_{L T}=-\sqrt{2}\left(X_{0 x}+X_{x 0}\right)
$$




$$
f_{T T}=-\left(X_{x x}-X_{y y}\right) \quad f_{L T}^{\prime}=i \sqrt{2}\left(X_{0 y}-X_{y 0}\right) \quad f_{T}^{\prime}=-i\left(X_{x y}-X_{y x}\right)
$$

where, $X_{\alpha \beta}=\operatorname{Tr}\left(J_{\alpha} \rho_{N} J_{\beta}\right)$, for $(\alpha, \beta=0, x, y)$.

Taking the initial nucleon density matrix $\rho_{N}=\frac{1}{2}(1+\underline{P} \cdot \underline{\sigma})$, where $\underline{P}$ is the nucleon polarisation and the nuclear current given by eqn. (2.21) :

$$
J_{0}=G_{E}, \quad J_{x}=i \frac{q}{2 M} G_{M} \sigma_{y}, \quad J_{y}=-i \frac{q}{2 M} G_{M} \sigma_{x}
$$

where $\mathrm{M}$ is the nucleon mass and $G_{E}$ and $G_{M}$ are the nucleon electromagnetic form factors. We get for free elastic scattering,

$$
\begin{array}{lll}
f_{L}=G_{E}^{2}, & f_{T}=\frac{q^{2}}{2 M^{2}} G_{M}^{2}, \quad & f_{L T}=0 \\
f_{T T}=0, & f_{L T}^{\prime}=-\sqrt{2} \frac{q}{M} G_{E} G_{M} P_{x}, & f_{T}^{\prime}=-\frac{q^{2}}{2 M^{2}} G_{M}^{2} P_{z}
\end{array}
$$




\section{Bibliography}

[1] G.Holzwarth. hep-ph/0201138.

[2] E.Rutherford. Phil. Mag., sixth series, 21:669, 1911.

[3] E.Rutherford. Phil. Mag., sixth series, 37:537, 1919.

[4] J.Chadwick. Proc. Roy. Soc., A136:692, 1932.

[5] R.Frisch and O.Stern. Z. Phys., 85:4, 1933.

[6] R.W.McAllister R.Hofstadter. Phys. Rev., 102:851, 1956.

[7] M.N.Rosenbluth. Phys.Rev., 79:615, 1950.

[8] R. G. Sachs. Phys. Rev., 126, 1962.

[9] Martin and Shaw. Particle Physics. Wiley, 1997.

[10] Reinhardt Greiner. Quantum Electrodynamics. Springer, 1994.

[11] D. Cheng and G. O’Neil. Elementary Particle Physics. Addison-Wesley.

[12] G. Hoehler et al. Nucl. Phys. B, 114:505, 1976.

[13] P. Mergell et al. Nucl. Phys. A, 596:367, 1996.

[14] G.Kubon et al. Phys. Lett. B, 524:26, 2002.

[15] S. Kopecky et al. Phys. Rev. Letts., 74:2427, 1995.

[16] L.L Foldy. Rev.Mod.Phys., 30:473, 1958.

[17] N.Isgur. Phys. Rev. Letts., 83:272, 1999.

[18] James J. Kelly. Phys. Rev. C, 66:065203, 2002. 
[19] A.L. Licht and A. Pagnamenta. Phys. Rev. D, 31:1156, 1970.

[20] C. Herberg et al. Eur. Phys. J. A, 5, 1999.

[21] S.Platchkov et al. Nuc. Phys., A510:740, 1990.

[22] T.Hamada and I.Johnson. Nucl. Phys., 34:382, 1962.

[23] E.Lomon and H.Feshbach. Rev. Mod. Phys., 39:611, 1967.

[24] R.Reid. Ann. Phys., 50:411, 1968.

[25] T.A. Griffy and L.I.Schiff. High Eenrgy Physics Vol.1. Burshop.

[26] M.Gourdin. Nuovo Cimento, 28:533.

[27] E.L. Lomon. Ann. of Phys., 125:309, 1980.

[28] H. Arenhovel et al. Z. Phys. A, 331:123, 1988.

[29] T.W. Donnelly and A.S. Raskin. Ann. Phys., 169:247, 1986.

[30] H.Schmieden. Eur. Phys. J. A, 1:427, 1998.

[31] S.Galster et al. Nuclear Physics, B32:221.

[32] H.Feshbach and E.Lomon. Rev. Mod. Phys., 39:611, 1967.

[33] I.McGee. Phys. Rev., 151:772, 1966.

[34] R.Schiavilla and I.Sick. Phys. Rev. C, 64:041002-1, 2001.

[35] T.Eden et al. Phys. Rev. C, C50:1749, 1994.

[36] I.Passchier et al. Phys. Rev. Letts., 82:4988, 1999.

[37] M. Ostrick et al. Phys. Rev. Lett. 83, 276, 83, 1999.

[38] R. G. Arnold et al. Phys. Rev. C, C23, 1981.

[39] A.I. Akhiezer and M.P. Rekalo. Sov. J. Part. Nucl., 4:277, 1973.

[40] H.Zhu. Phys. Rev. Letts., 87:081801, 2001.

[41] J.Becker et al. Eur. Phys. J., A6:329, 1999.

[42] D.Rohe et al. Phys. Rev. Letts, 83:4257, 1999. 
[43] J.Bermuth et al. Phys. Letts. B, 564:199, 2003.

[44] J.Golack et al. Phys. Rev. C, C63:034006, 2001.

[45] H.Anklin et al. Phys. Rev. Lett. B, 336:313, 1994.

[46] H.Anklin et al. Phys. Rev. Lett.B, 428:248, 1998.

[47] E.E.W.Bruins et al. Phys. Rev. Lett, 75:21, 1995.

[48] W.Xu et al. Phys. Rev. Letts, 85:2900, 2000.

[49] W.Xu et al. Phys. Rev.C, 67:012201(R), 2003.

[50] A.Lung et al. Phys. Rev. Lett., 70:718, 1993.

[51] K.I.Blomqvist et al. The three-spectrometer facility and the Mainz-microtron MAMI. Nucl. Instr. Meth. A, 403:263, 1998.

[52] M.Seimetz. Messung des elektrischen Formfaktors des Neutrons bei $Q^{2}=0.3$ und $0.6 \frac{\mathrm{GeV}^{2}}{c^{2}}$. PhD thesis, Johannes-Gutenberg-Universitaet,Mainz, 2003.

[53] K. Aulenbacher et al. Nucl. Instr. Meth. A, 391, 1997.

[54] H. Herminghaus et al. In Proc. Linear Accelerator Conference, Albequerque, 1990.

[55] A1 collaboration. The liquid Hydrogen/Deuterium target. http://wwwa1.kph.uni-mainz.de/A1/target.html.

[56] L.Wolfenstein and J.Ashkin. Phys. Rev., 85:947, 1952.

[57] L.Wolfenstein. Ann.Rev.Nuc.Sci., 6:43, 1956.

[58] SAID. http://said-hh.desy.de.

[59] T. N. Taddeucci et al. Nucl. Instr. Meth. A, 241, 1985.

[60] Weis Distler, Merkel. Data Acquisition and Analysis. Technical report, A1 collaboration, MAMI.

[61] R.Watson. A Measurement of the Neutron Electric Form Factor. PhD thesis, University of Glasgow, 1997.

[62] Geant4 Collaboration. http://wwwasd.web.cern.ch/wwwasd/geant4/. 
[63] Rene Brun and Fonz Rademakers. ROOT, http://root.cern.ch.

[64] The C++ Programming Language. Addison Wesley, 1995.

[65] M.Bernheim et al. Nuc. Phys, A365:349, 1981.

[66] R.A. Cecil et al. Nucl. Instr. Meth., 161:439, 1979.

[67] D.Dutta et al. Phys. Rev. C, 61:061602-1, 2000.

[68] G.V. O’Rielly et al. Nucl. Instr. Meth. in Phys. Res. A, 368:745, 1996.

[69] R. Madey et al. Nucl. Instr. Meth., 151:445, 1978.

[70] C.D. Zafiratos J. Czirr, D.R. Nygren. Nucl. Instr. Meth., 31:226, 1964.

[71] F. Klein and M. Seimetz. Spatial Calibration of the A1 Neutron Polarimeter. Private Communication.

[72] F. Klein. Kalibration des Neutronenpolarimeters. Master's thesis, Johannes Gutenberg-Universitat Mainz, 2001.

[73] W.Braunschweig et al. Nucl. Instr. Meth, 134:261, 1976.

[74] V. Bargmann. Phys. Rev. Lett., 2:435, 1959.

[75] M. Seimetz. The Spin Precession Magnet of the A1 Neutron Polarimeter. Private Communication.

[76] David R.Giebink. Phys. Rev. C, 32:502, 1985.

[77] F.James. Minuit, http://www.asdoc.web.cern.ch/wwwasdoc/minuit/minmain.html.

[78] D.L. Prout et al. Phys. Rev. C, 52, 1995.

[79] R. Rosenfelder. Phys. Letts., 79B:15, 1978.

[80] V.R. Pandharipande et al. Phys. Rev. C, 49:789, 1994.

[81] J. Wambach. Phys. Rev. C, 46:807, 1992.

[82] M. Viviani A De Pace. Phys. Rev. C, 48:2931, 1993.

[83] K.H. Hicks. Phys. Rev. Letts., 47:260, 1993.

[84] C.J. Horowitz and D.P. Murdock. Phys. Rev. C, 37:2032, 1988. 
[85] M.K. Jones et al. Phys. Rev. Lett., 84:1398, 2000.

[86] J.Friedrich Th. Walcher. accepted Eur.Phys.J. A, hep-ph/0303054.

[87] S.J.Brodsky and G.P.Lepage. Phys. Rev. D, 22:2157, 1981.

[88] Pankaj Jain and John P. Ralston. The Proton Electromagnetic Form Factor $F_{2}$ and Quark Orbital Angular Momentum. In Proceedings of the Workshop QCD 2002.

[89] O.Gayou et al. Phys. Rev. Letts, 88:092301-1, 2002.

[90] D.Drechsel P.Mergell, U.G.Meissner. Nucl. Phys. A, 596:367, 1996.

[91] R.F.Wagenbrunn et al. Phys. Lett. B, 511:33, 2001.

[92] S.Simula F.Cardelli. Phys. Rev. C, 62:065201, 2000.

[93] M.F.Gari and W.Krumpelmann. Phys. Letts., B274:159, 1992.

[94] M.F.Gari and W.Krumpelmann. Phys. Letts., B173:10, 1986.

[95] B.Wojtsekhowski. Measurement of the Neutron Electric Form Factor $G_{E}^{n}$ at high $Q^{2}$. JLAB proposal, 2001. 